\title{
Equilibrium and Non-Equilibrium Ultrafast Carrier Transport and Dynamics in Chalcopyrite Semiconductors
}

\author{
Rishmali Thanuja Sooriyagoda \\ West Virginia University, rtsooriyagoda@mix.wvu.edu
}

Follow this and additional works at: https://researchrepository.wvu.edu/etd

Part of the Condensed Matter Physics Commons

\footnotetext{
Recommended Citation

Sooriyagoda, Rishmali Thanuja, "Equilibrium and Non-Equilibrium Ultrafast Carrier Transport and Dynamics in Chalcopyrite Semiconductors" (2021). Graduate Theses, Dissertations, and Problem Reports. 8097.

https://researchrepository.wvu.edu/etd/8097

This Dissertation is protected by copyright and/or related rights. It has been brought to you by the The Research Repository @ WVU with permission from the rights-holder(s). You are free to use this Dissertation in any way that is permitted by the copyright and related rights legislation that applies to your use. For other uses you must obtain permission from the rights-holder(s) directly, unless additional rights are indicated by a Creative Commons license in the record and/ or on the work itself. This Dissertation has been accepted for inclusion in WVU Graduate Theses, Dissertations, and Problem Reports collection by an authorized administrator of The Research Repository @ WVU. For more information, please contact researchrepository@mail.wvu.edu.
} 


\title{
Equilibrium and Non-Equilibrium Ultrafast Carrier Transport and Dynamics in Chalcopyrite Semiconductors
}

\section{Rishmali Thanuja Sooriyagoda}

\author{
Dissertation submitted \\ to the Eberly College of Arts and Sciences \\ at West Virginia University \\ in partial fulfillment of the requirements for the degree of \\ Doctor of Philosophy \\ in \\ Physics
Alan D. Bristow, Ph.D., Chair
Matthew Johnson, Ph.D.
Tudor Stanescu, Ph.D.
Konstantinos Sierros, Ph.D. \\ Department of Physics and Astronomy \\ Morgantown, West Virginia \\ 2021
}

Keywords: Ultrafast Optics, Chalcopyrite semiconductors, Terahertz TimeDomain Spectroscopy, AC Conductivity, Electron-Lattice Interactions, Electronic

Transport, Terahertz Time-Resolved Spectroscopy, AC Photoconductivity Copyright 2021 Rishmali Thanuja Sooriyagoda 


\title{
Abstract \\ Equilibrium and Non-Equilibrium Ultrafast Carrier Transport and Dynamics in Chalcopyrite Semiconductors
}

\author{
Rishmali Thanuja Sooriyagoda
}

Chalcopyrite crystals in the II-IV-V $\mathrm{V}_{2}$ family have received significant interest due to their high nonlinearity, composition-tunable bandgaps, wide transparency windows, and high damage threshold. These semiconductors have been explored for electromagnetic (EM) screening, spintronic and photovoltaic applications, making them good optical and optoelectronic materials. This thesis uses terahertz spectroscopy to understand optical, electronic, and vibrational dynamical processes in $\mathrm{CdGeP}_{2}, \mathrm{ZnGeP}_{2}$ and $\mathrm{CdSiP}_{2}$ chalcopyrite semiconductors.

We have employed Terahertz time-domain spectroscopy to investigate temperaturedependent ground-state properties of bulk chalcopyrite crystals that can be related to electronic transport and electron-lattice interactions. The complex spectra provide refraction and absorption from which electron-phonon coupling and average phonon energies are extracted. AC conductivity spectra provide carrier densities and electron scattering times, the temperature dependence of which are associated with unintentional shallow dopants. Temperature dependence of the scattering time is converted into carrier mobility and modeled with microscopic transport mechanisms such as polar optical phonon, acoustic phonons, deformation potential, ionized impurity, and dislocation scattering. Hence, analysis links the terahertz response to properties that can only be obtained using high-frequency AC Hall-effect measurements, Raman scattering, and conventional continuous-wave spectroscopy.

Ultrafast photoexcited charge-carrier dynamics are investigated using time-resolved terahertz spectroscopy. Analysis of the differential $\mathrm{THz}$ transient reveals a two-component exponential relaxation, with decay times that increase with pump fluence due to state filling. Inverting the transients recaptures the canonical rate-equation for the system and from which the recombination dynamics are attributed to the entire excitation range. To support the recombination information, $\mathrm{THz}$ photoconductivity determines the excited-state carrier transport as a function of temperature. Both elevated temperatures and increased excitation density decreases excited-state carrier mobility and are attributed to carrier-carrier interactions and scattering from phonons.

These model semiconductors, which have application in photonics, allow for further demonstration of pulsed-THz-based spectroscopy to capture both charge transport and dynamics. Additionally, several charge transport and carrier-lattice properties for $\mathrm{CdGeP}_{2}, \mathrm{ZnGeP}_{2}$, and $\mathrm{CdSiP}_{2}$ have been reported that can assist with the design and optimize of devices that comprise them. 
Dedication

To my parents and my husband 


\section{Acknowledgments}

First and foremost, I would like to express my deepest gratitude to my advisor Dr. Alan D. Bristow for his guidance, tremendous support, and encouragement over the last five years. I am very grateful to Dr. Bristow for the freedom given to me that helped me grow as a researcher. I consider myself very lucky to have Dr. Bristow as my advisor and mentor. I would also like to thank my committee members: Dr. Matthew Johnson, Dr. Tudor Stanescu, and Dr. Konstantinos Sierros for their time and support during my oral qualifying exam and throughout this dissertation.

Many thanks to our collaborators Dr. Peter G Schunemann and Dr. Kevin T Zawilski at BAE for providing us Chalcopyrite samples.

I am very grateful to all my lab mates for their support and great company. Especially I would like to thank Chathuranaga Piyathilaka for helping me with my experiments and useful conversations on research projects. Many thanks to Jack Powers for his help on the inversion analysis. I want to extend my thanks to Dr. Tess R. Senty and Brandon Yost for their help with the experimental setup. I would also like to thank all my friends for their support and the great times we had.

A special thanks to my parents and brother for their unconditional love, unwavering support, and encouragement throughout my life. They are the biggest inspiration of my life. Finally, I would like to thank my husband for his love, understanding, and patience. He always there for me throughout the ups and downs of my life. I never would have been able to come this far without his support.

Rishmali Thanuja Sooriyagoda 


\section{Table of Contents}

Abstract.................................................................................................................................................. ii

Dedication ....................................................................................................................................... iii

Acknowledgments ................................................................................................................................. iv

Table of Contents ......................................................................................................................

Chapter 1. Introduction .......................................................................................................................................... 1

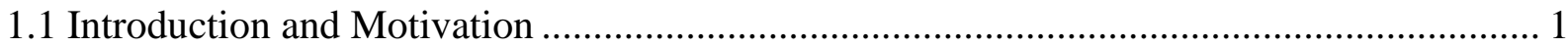

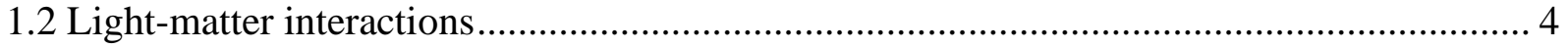

1.3 Ultrafast Spectroscopy ……………………………................................................ 7

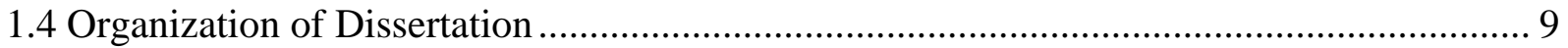

Chapter 2: Experimental Techniques .......................................................................................... 10

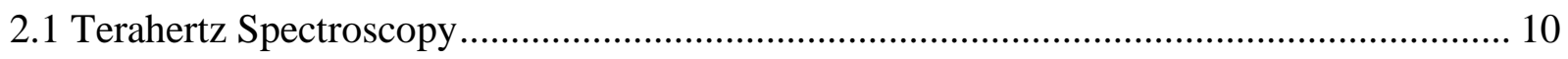

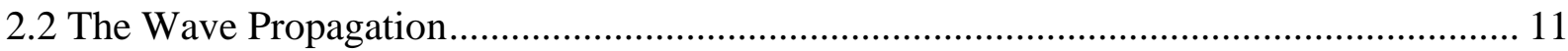

$2.3 \mathrm{THz}$ sources and optical Rectification ......................................................................... 13

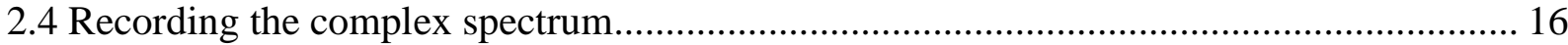

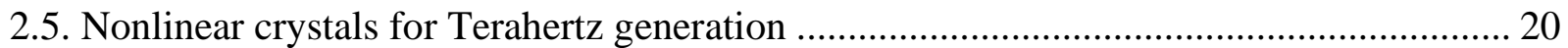

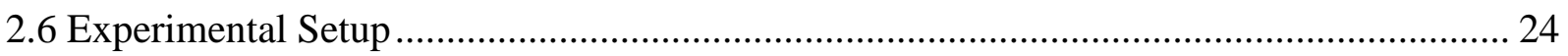

2.7. Terahertz Time-Domain Spectroscopy (THz-TDS) ……………………….................. 27

2.8 Time-Resolved Terahertz Spectroscopy (TRTS)............................................................. 30

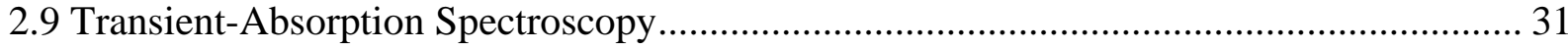

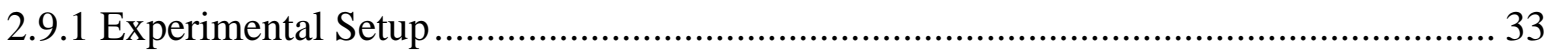

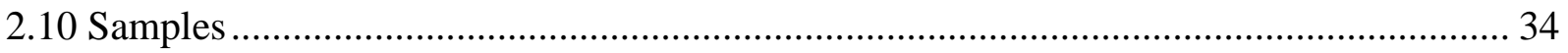

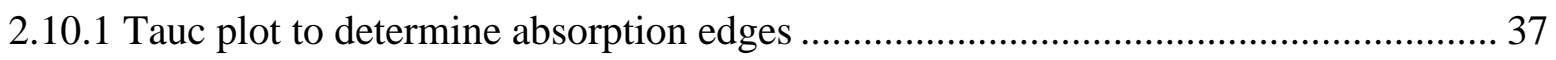

Chapter 3: Ground-State AC Conductivity ............................................................................... 39

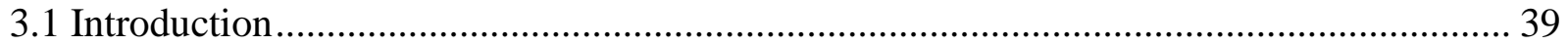

3.2 Extracting Complex Material Parameters ......................................................................... 40

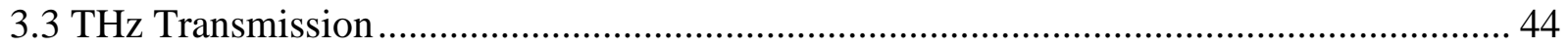

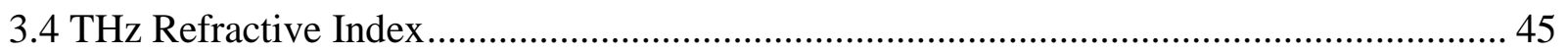

3.4.1 The Temperature Dependence of the Refractive Index .............................................. 47

3.4.2 The Band Gap Dependence of the Refractive Index ................................................. 50

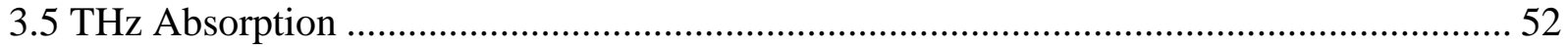




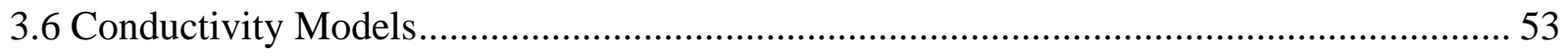

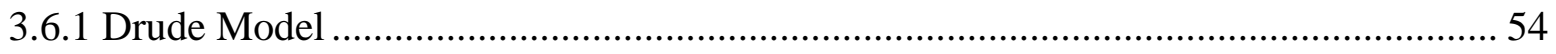

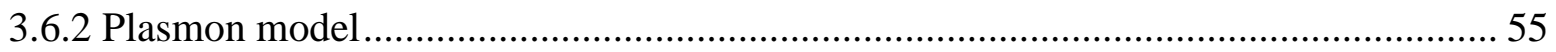

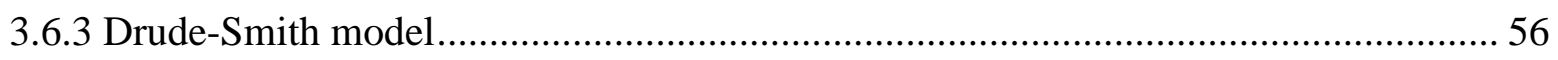

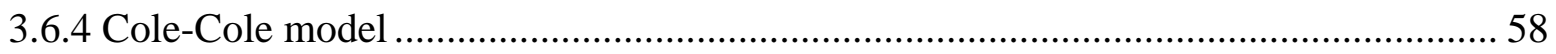

3.7 Conductivity Response of Chalcopyrite crystals .............................................................. 58

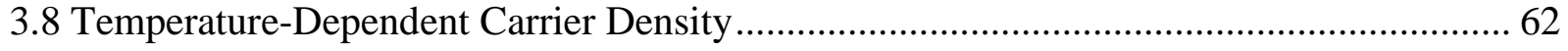

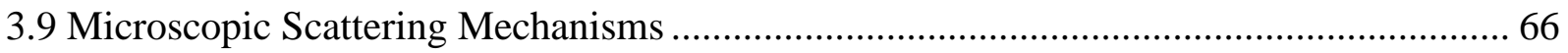

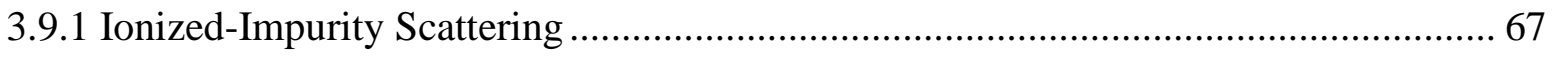

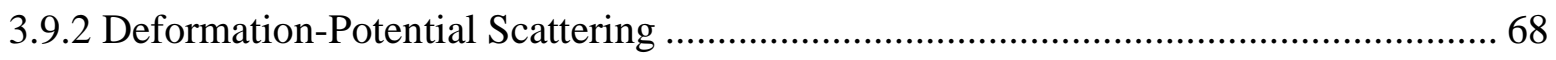

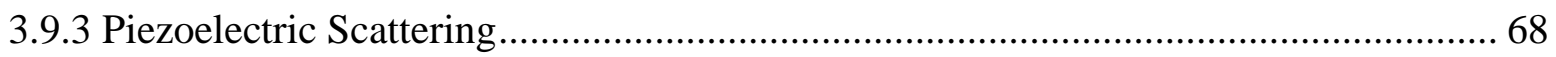

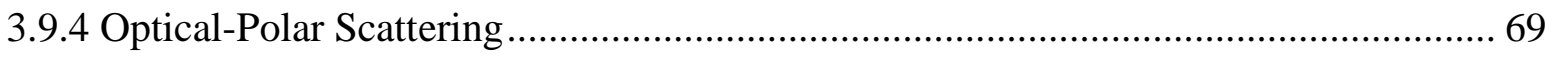

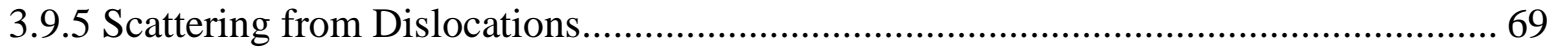

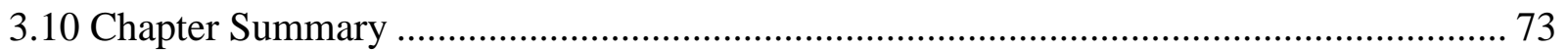

Chapter 4: Non-Equilibrium Carrier Dynamics ............................................................................. 74

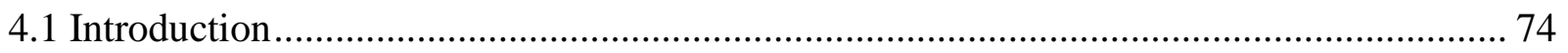

4.2 Charge-Carrier Recombination ................................................................................... 75

4.3 Pump-probe measurements of Chalcopyrite crystals......................................................... 78

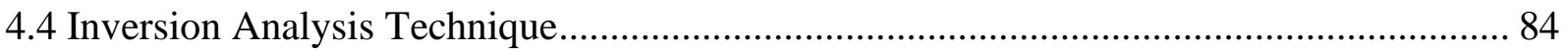

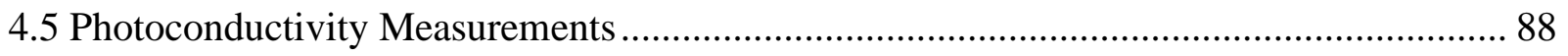

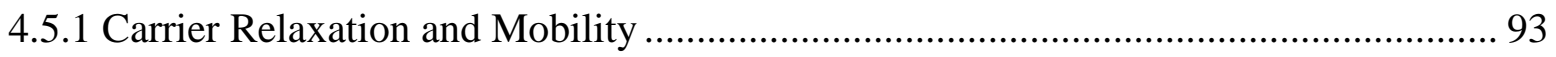

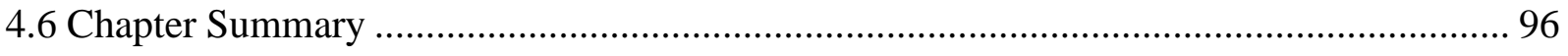

Chapter 5: Conclusion ......................................................................................................................... 98

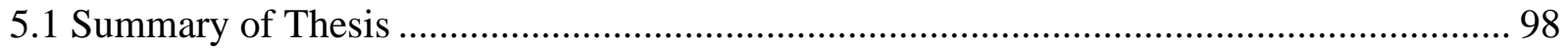

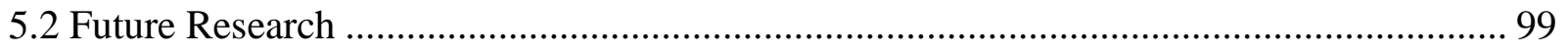

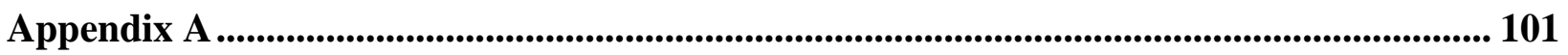

A.1 Transient Absorption Spectroscopy on Carbon Nitride Crystals .................................... 101

References.............................................................................................................................................................. 105 


\section{Chapter 1. Introduction}

\subsection{Introduction and Motivation}

Modern electronics and optoelectronics are nearly entirely based on advanced semiconductor technology. Semiconductor materials and devices play a major role in today's world due to their wide range of applications. The first documented observation of a semiconductor effect was reported by Michael Faraday in 1833 [1]. He discovered that the conductivity of silver sulfate increases with temperature, which was contrary behavior compared to the metals. By 1935, selenium rectifiers and silicon point-contact diodes were used as radio detectors [2]. In 1947, John Bardeen, Walter H. Brattain, and William B. Shockley invented a germanium point-contact transistor at Bell Telephone Laboratories [3]. They received the Nobel Prize in Physics in 1956 for their discovery. This invention of transistors marked the beginning of a new era in which semiconductors became ever more important. It was one of the most technical developments of the $20^{\text {th }}$ century. Since then, semiconductors have been extensively used in information technology, computer industry, medicine, and every facet of human existence, where those technologies can be employed from digital art to remote-controlled aircraft.

In many applications, optoelectronic devices play an essential role. Optoelectronics is the technology of electronic devices which interact with light. Optoelectronics brings optics and electronics together within a single device. They convert electrical energy into the light for example in LEDs and semiconductor lasers. They can also detect light and convert light into electrical currents, for example in photodiodes, photodetectors, and photovoltaic cells. The modern era of semiconductor optoelectronics began in the early 1960s with the development of LED from p-n junction in GaAs [4]. The development of photonic devices significantly increases since the invention of the laser in 1960 [5]. Most electronic devices are based on silicon due to their low cost and versatility. However, silicon has an indirect band gap and very low light-emitting efficiency. Whereas most optoelectronic devices are produced using III-V semiconductors such as $\mathrm{GaAs}, \mathrm{InP}, \mathrm{GaN}$, and GaSb and their alloys because of their direct bandgap, high electron mobility, and low exciton binding energy [6-17]. Semiconductors such as $\mathrm{GaAs}, \mathrm{LiNbO}_{3}, \beta$ - barium borate (BBO), and $\mathrm{KNbO}_{3}$ are widely employed for nonlinear EM frequency mixing [18-26]. Moreover, semiconductors like GaAs and $\mathrm{GaP}$ are used for frequency modulation applications [27,28]. 
One semiconductor class that has been interesting to the optics community is chalcopyrite crystals due to their strong nonlinearity, wide bandgaps, broad transparency windows, and high damage threshold [29-32]. Chalcopyrite semiconductors are ternary compounds which have a tetragonal structure with $\mathrm{ABC}_{2}$ Stoichiometry [33]. The name chalcopyrite comes from the cooper iron sulfide mineral $\left(\mathrm{CuFeS}_{2}\right)$ which formed naturally. Chalcopyrite crystal is originated from two Greek words "chalkos" meaning copper and "pyrites" meaning strike fire. There are two types of chalcopyrite semiconductors: I-III-V $1_{2}$ and II-IV-V 2 compounds which belongs to the $I 4 \overline{2} d$ space group and $\overline{4} 2 m$ point group symmetry [33].

In 1953, Hahn et al. synthesized and characterized the first I-III-V $V_{2}$ crystal with a chalcopyrite structure [34]. In 1954, Goodman and Douglas predicted the possibility of having semiconductors in the II-IV-V $V_{2}$ form [35,36]. Since then, chalcopyrite crystals have been extensively studied due to their potential for wide-ranging applications. Originally investigated for their low thermal conductivities, now they are explored for electromagnetic (EM) screening, spintronic [37,38], and photovoltaic applications [39]. One area where they important is optoelectronic devices, such as optical parametric oscillators, light-emitting diodes (LEDs), laser diodes (LDs), and nonlinear optical (NLO) and optical modulator devices. [40-46]. They offer an excellent platform in nonlinear optics for generating electric fields spanning the visible, infrared, and terahertz (THz) spectral regions. Large-area chalcopyrite crystal growth offers the opportunity to produce good optical or optoelectronic materials and devices especially frequency converters in solid state-based tunable laser systems [47-51]. Moreover, some chalcopyrite crystals are predicted to show a topological insulating phase in their native state [52] and show possibility for high levels of magnetic-impurity doping [37].

Cadmium germanium diphosphide $\left(\mathrm{CdGeP}_{2}\right.$ or $\left.\mathrm{CGP}\right)$, zinc germanium diphosphide $\left(\mathrm{ZnGeP}_{2}\right.$ or $\left.\mathrm{ZGP}\right)$, and cadmium silicon diphosphide $\left(\mathrm{CdSiP}_{2}\right.$ or $\left.\mathrm{CSP}\right)$ are chalcopyrite semiconductor crystals that belong to the II-IV-V $V_{2}$ family. These three chalcopyrite crystals received great interest in optical applications due to their excellent nonlinear figure of merit $\left(d^{2} / n^{3}\right)$ [53] where $d$ is the nonlinear tensor element and $n$ is the refractive index. Also, they offer a broad transparency window compare to traditional nonlinear oxide crystals such as lithium niobite $\left(\mathrm{LiNbO}_{3}\right)$ or potassium titanyl phosphate (KTP) [53] as shown in Figure 1.1. CGP, ZGP, and CSP have been recognized as promising NLO crystals since the 1970s. At that time crystals 
were grown using the halogen-assisted vapor transport method. However, the size and quality of the crystals were inadequate to measure NLO properties and produce devices [54]. The most common drawbacks during the large crystal growth process were cracking and growth of multiple crystals. Moreover, defects which were introduced during the growth process resulted in decreased transparency due to absorption and scattering. The horizontal-gradient-freeze (HGF) growth technique has been developed to overcome these drawbacks by Zawilski and Schunemann [55,56]. High optical quality large crystals have been grown using this method. This breakthrough in the crystal growth technique has brought these materials to the forefront of optoelectronics.

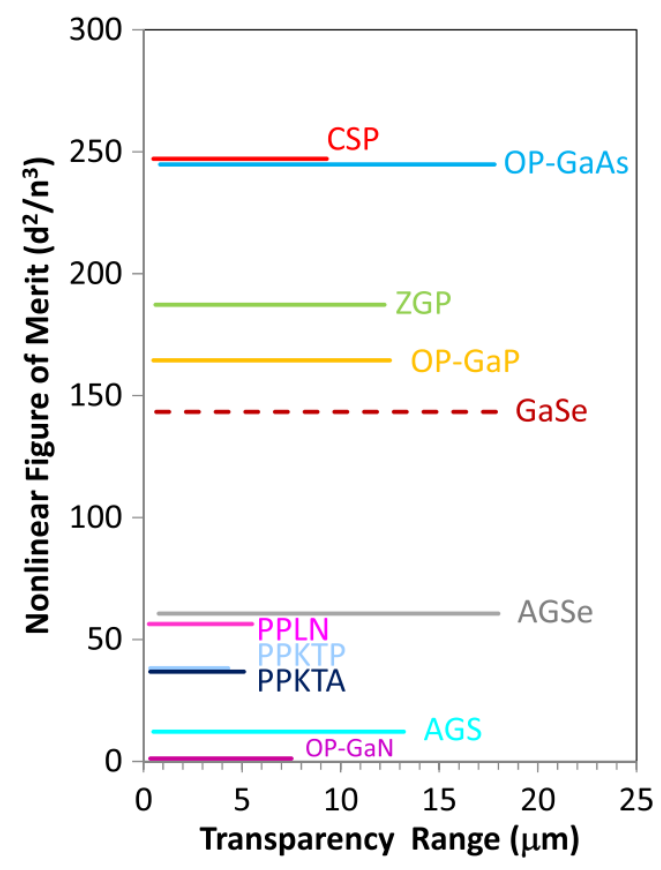

Figure 1.1. Nonlinear figure of merit versus transparency windows Figure adapted with permission from [53] @ The Optical Society

Many studies have been carried out on CGP, ZGP, and CSP chalcopyrite crystals to study electrical and optical properties. A detailed review of chalcopyrite compounds was given in a book by Shay and Wernick [33]. Miller et al. reported the measurements of Hall effects and conductivities of CGP and ZGP crystals using the Van-der-Pauw technique [57]. Several theoretical studies have investigated the electronic structure, dielectric and elastic properties of CGP, ZGP, and CSP chalcopyrite crystals [58-62]. Point defects in large single crystals of ZGP 
and CSP crystals have been identified using electron paramagnetic resonance (EPR) and optical absorption experiments [63-67]. Bettini et al. have determined the phonon spectra of CGP and ZGP by Raman scattering at room and liquid nitrogen temperatures [68]. Raman scattering has studied phonon spectra in CSP crystal by Sho Shirakata [69]. Zhong et al. have investigated the linear optical properties of ZGP crystal in the terahertz range [70]. They have measured the refractive index and absorption coefficient using terahertz spectroscopy and Fourier transform infrared spectroscopy (FTIR). Carnio et al. have reported the optical properties of CSP crystal in the $\mathrm{THz}$ frequency regime [48,71]. Nonlinear optical measurements of these crystals have been intensively studied by many researchers $[29,32,49,72-74]$.

Although optical properties are generally known in CGP, ZGP, and CSP chalcopyrite crystals, there have been no prior systematic studies of temperature-dependent $\mathrm{THz}$ dispersion and AC conductivity. Also, there is still much to understand about models of conduction and their effect at $\mathrm{THz}$ frequencies. Hence, chalcopyrite crystals are a model system for exploring temperature-dependent Terahertz time-domain spectroscopy (THz-TDS) acquisition of microscopic electronic transport phenomena by applying models that have traditionally been applied in Hall-effect measurements as a function of temperature. This approach overcomes the poor experimental reliable of Hall measurements of chalcopyrite crystals arising from their low mobility. Moreover, various excited-state dynamics should be explored to develop and optimize optoelectronic devices. According to the best of our knowledge, there have been not yet been studied the non-equilibrium carrier dynamics of CSP. ZGP and CSP chalcopyrite crystals. Hence the central motivation of this thesis is to investigate temperature-dependent ground-state and excited-state carrier dynamics and transport properties of CSP, ZGP, and CSP chalcopyrite semiconductors using terahertz spectroscopy.

\subsection{Light-matter interactions}

Understanding light-matter interactions is essential for fundamental knowledge as well as for developing, optimizing, and designing devices. Interaction between light and matter plays a significant role in spectroscopy, lasers, LEDs, X-ray sources, solar cells, photodiodes, quantum information processing, etc. Light can interact with matter in many ways such as absorption, reflection, transmission, scattering, etc. Light-matter interactions were classically described by 
Hendrik Lorentz in the 1900s using Maxwell's equations. In this model, the electron is bound to the nucleus of the atom by a spring-like force $[75,76]$. An applied oscillatory electromagnetic field interacts with the electron. Then electron oscillates about the nucleus and undergoes forced motion as shown in figure 1.2. In this model, the movement of the nucleus is neglected. Also, Lorentz assumed that the core electrons are tightly bound and only valance electrons response to the external field. Hence the optical properties of a solid are primarily determined by the valance electrons. This model valid for most of the materials. With the introduction of quantum mechanics, many theories were developed to describe the light-matter interactions [77].

In the ground state, electron (and hole) populations in a semiconductor are described by Fermi-Dirac statics. Meanwhile, the lattice vibrations are described by the phonons. Phonons are Bosons and Bose-Einstein distribution is used to describe them [78]. Energy and momentum are interchanged via carrier-carrier and carrier-phonon interactions, which keep distributions at a common temperature. Hence average momentum of the carrier and phonon system is zero and their average energy is related to their relative temperatures [79]. Moreover, the lifetime of the scattering process on a solid is affected by the carrier-carrier, carrier-phonon interactions. Typically, carrier-carrier scatterings are on the order of $10^{-15}-10^{-12} \mathrm{~s}$ and carrier-phonon scatterings are on the order of $10^{-12} \mathrm{~s}$.

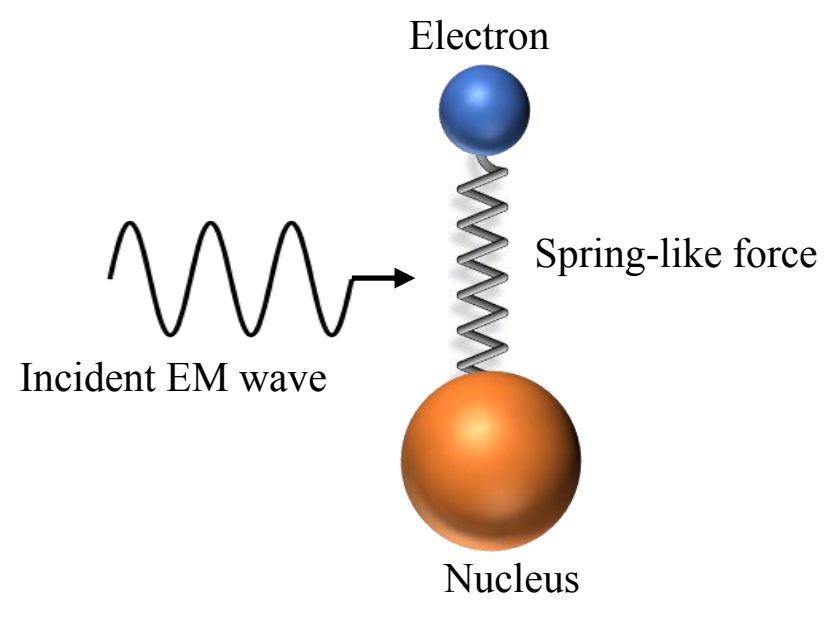

Figure 1.2. The classical Lorentzian model 
Excitation of semiconductors with an optica1 pulse results in a non-equilibrium condition. Immediately after the photoexcitation, the energy distribution of the carriers cannot be explained using Fermi-Dirac distribution with a defined temperature. This is called a non-thermal regime. To study this regime, it requires a time resolution of about $<100 \mathrm{fs}$. After a few hundred femtoseconds a Fermi-Dirac distribution is reached via carrier-carrier and carrier-phonon scattering and achieved a hot carrier temperature, which is higher than the lattice temperature. This is known as the hot-carrier regime. Understanding of non-thermal and hot-carrier regimes provides information about carrier-carrier and carrier-phonon scattering. Then carriers cooled to the lattice temperature by transferring its energy to the lattice via phonon scattering and recombination. This process creates non-equilibrium (hot) phonons. Then carriers further relax via radiative and nonradiative recombination mechanisms. Table 1.1 shows the typical time scale for different processes in solid $[79,80]$.

Table 1.1. The typical time scale for fundamental processes in semiconductors $[79,80]$.

\begin{tabular}{|c|c|c|}
\hline Time & Microscopic process & Characteristic time (s) \\
\hline & Lattice heat diffusion $(11 \mu \mathrm{m})$ & $\sim 10^{-8} s$ \\
\hline & Radiative Recombination & $\geq 10^{-9} s$ \\
\hline & Auger recombination (carrier density $10^{20} \mathrm{~cm}^{-3}$ ) & $\sim 10^{-10} \mathrm{~s}$ \\
\hline & Carrier diffusion $(0.1 \mu \mathrm{m})$ & $\sim 10^{-11} \mathrm{~s}$ \\
\hline & Optical phonon-acoustic phonon interactions & $\sim 10^{-11} \mathrm{~s}$ \\
\hline & Carrier-optical phonon thermalization & $\geq 10^{-12} \mathrm{~s}$ \\
\hline & Intravalley scattering & $\geq 10^{-13} \mathrm{~s}$ \\
\hline & Intervalley scattering & $\geq 10^{-14} \mathrm{~s}$ \\
\hline & Carrier-carrier scattering & $10^{-15}-10^{-12} s$ \\
\hline & Electron correlation Time & $<10^{-12} s$ \\
\hline
\end{tabular}




\subsection{Ultrafast Spectroscopy}

As shown in Table 1.1, most of the microscopic processes in a semiconductor occur on a picosecond or even a femtosecond time scale. It is not possible to achieve these time resolutions using conventional methods such as fast photodiodes and electrical measurements. Conventional spectroscopic methods are energy or frequency-resolved measurements. They have been used to study electronic band structure, phonons, defects, impurities, interfaces, etc. These measurements provide information about the time-resolved kinetic processes which are statistical. Whereas ultrafast spectroscopy enables us to directly probe the dynamics of the system.

Short-pulsed lasers are essential for ultrafast spectroscopy. Traditional lasers like ruby lasers normally operate with nanoseconds or even longer pulses [81,82]. Mode-locking is a technique to produce ultrashort pulses. Different modes in a simple laser resonator oscillate with independent frequency and random mode. In mode-locking, the phase of the longitudinal modes is locked to each other [83]. If adequate longitudinal modes are locked together with small phase differences, shorter pulses can be obtained to satisfy Fourier time-energy limits. There are various ways to achieve mode-locking such as active mode-locking are passive mode-locking [83]. In 1965, Mocker and Collins first demonstrated passive mode-locking of the ruby laser [84]. Several months later DeMaria and coworkers generated the first optical pulses in the picosecond range using passively mode-locked Nd:glass laser [85]. These lasers produced pulses with a few tens of picosecond pulse duration at a repetition rate of $1 \mathrm{~Hz}$. One of the limitations of mode-locked $\mathrm{Nd}$ : glass laser is that it could not time-resolved processes that occur on sub-picosecond and femtosecond time scales. In 1974, Shank and Ippen, first generated the optical pulses shorter than 1 ps using a cavity dumped and passively mode-locked rhodamine-6 G dye laser [86]. In cavity dumped lasers, energy builds up in a resonator, then quickly releasing the power as an optical pulse [87]. In 1982, Titanium-doped sapphire (Ti: sapphire) laser was invented by Moulton [88]. The evolution of ultrafast laser has been significantly accelerated since the discovery of Ti: sapphire laser. They have become popular among the laser community because it allows generating femtosecond pulses with its outstanding mode-locking characteristics. Since then, new types of lasers with higher efficiency, shorter pulse duration, and broader spectrum range have been commercialized. Figure 1.3 shows ultrafast laser focused intensity as a function of time 


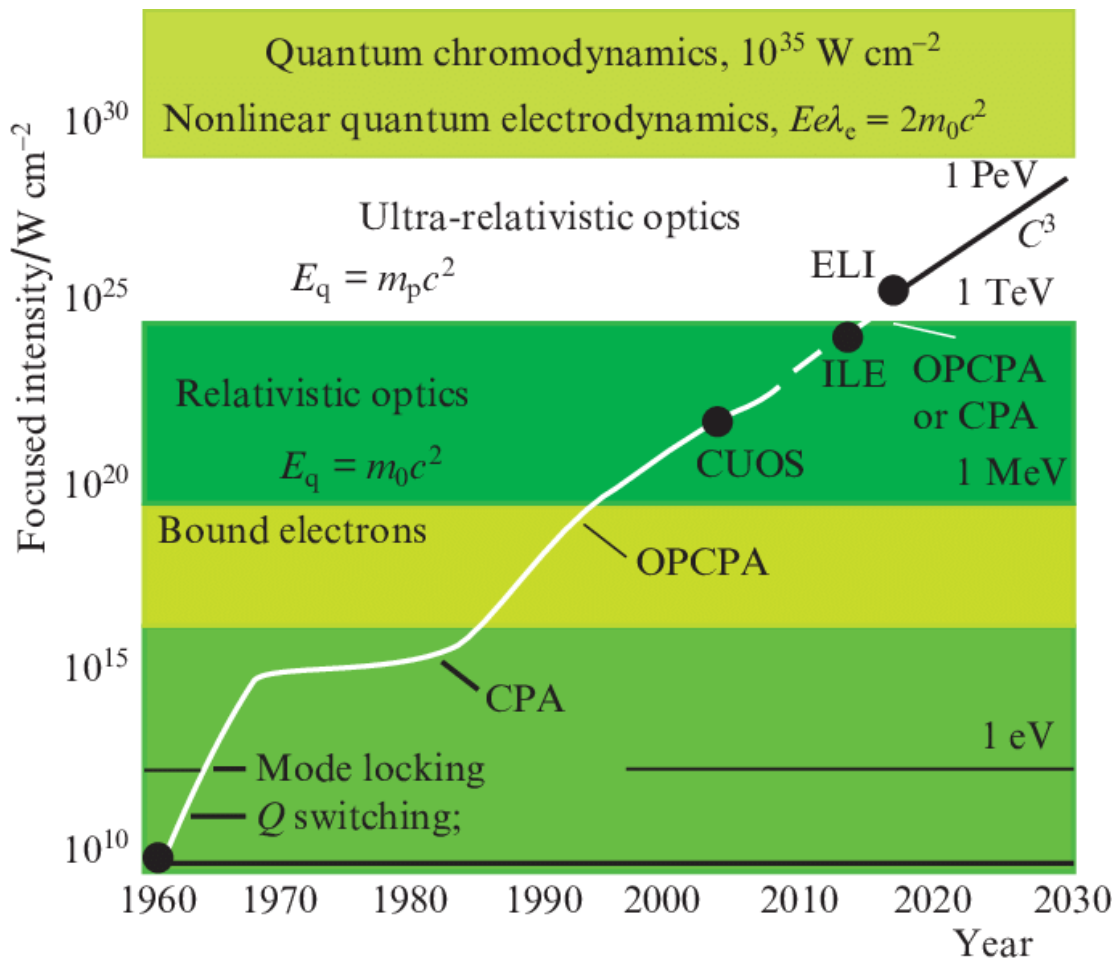

Figure 1.3. History of focused laser intensity. The figure is taken from [89].

In this dissertation, a Ti:sapphire laser amplifier is used to perform the experiments. Here ultrashort pulses are generated by chirped pulse amplification (CPA). The seed optical pulse with $80 \mathrm{MHz}$ repetition rate with $300 \mathrm{~mW}$ power at $800 \mathrm{~nm}$ central wavelength is generated using a mode-locked Ti:sapphire laser, pumped with a green CW diode laser. Then the seed pulse is stretched 10,000 times in order to reduce the power per unit time. The Ti:sapphire gain medium is pumped with a diode-pumped, Q-switched Nd:YLF laser with central wavelength at $527 \mathrm{~nm}$. The selected and stretched seed pulses made multiple passes through the regenerative amplifier. Then amplified pulses are directed into a compressor to shorten the amplified pulses and provide [30]. dispersion compensation. When pulses propagate through dispersive media such as Ti:sapphire crystal and optical elements, pulse broadening occurs. This dispersion can be compensated by prechipping the pulses [31,32].

The fast development of ultrafast lasers in the last 60 years enabled scientists to perform various ultrafast spectroscopic techniques such as pump-probe spectroscopy, Terahertz spectroscopy, time-resolved luminescence, ultrafast polarization spectroscopy, four-wave mixing 
spectroscopy, etc. These ultrafast spectroscopic techniques have been employed to study nonlinear properties, novel physical phenomena, transport dynamics [90,91], and other processes including the dynamics of free carriers [92-94], excitons $[95,96]$ and phonons $[97,98]$ in both the coherent and incoherent regimes.

\subsection{Organization of Dissertation}

The rest of the dissertation is organized as follows. Chapter 2 presents the basics principle of the experimental techniques and the basic properties of the samples. Firstly, the physics of the nonlinear optics involved in the $\mathrm{THz}$ generation and detection are discussed. Then fundamental principles and the detailed experimental setup of terahertz time-domain spectroscopy (THz-TDS), Terahertz time-resolved spectroscopy (TRTS), and pump-probe spectroscopy are introduced. Finally, the basics properties of the samples are discussed including the Tauc plots to determine the bandgap of samples.

Chapter 3 presents the temperature-dependent THz-TDS measurements of CGP, ZGP, and CSP bulk chalcopyrite semiconductors in the terahertz range. In this chapter, temperaturedependent refraction, absorption, electron-phonon coupling, AC conductivity, and mobility are determined. The measured optical and electronic properties are significantly influenced by the temperature that affects the underlying carrier scattering mechanism. This chapter will also discuss the specifics of data analysis, different conduction models, and various scattering mechanisms.

Chapter 04 discuss temperature-dependent nonequilibrium carrier dynamics, and complex AC photoconductivity of CGP, ZGP, and CSP crystal measured using TRTS. Analysis of the differential $\mathrm{THz}$ transient reveals a two-component exponential relaxation. Inversion analysis recaptures the canonical rate-equation for the system and from which the recombination dynamics are attributed to the entire excitation range. Carrier density and scattering time for various temperatures are obtained by fitting the photoexcited conductivity response with the Drude-Smith model. This detailed understanding of carrier dynamics and photoconductivity is crucial to potential applications of chalcopyrite crystals. This chapter will also present a theoretical background needed to understand the various recombination mechanisms. Finally, a summary and outlook follow in Chapter 5. 


\section{Chapter 2: Experimental Techniques}

\subsection{Terahertz Spectroscopy}

Terahertz $(\mathrm{THz})$ radiation lies between the microwave and the infrared regions of the electromagnetic spectrum. THz range roughly defined as frequencies $v=0.1-10 \mathrm{THz}$ which corresponds to photon energies $E=0.4-40 \mathrm{meV}$, or wavelengths $\lambda=3-0.03 \mathrm{~mm}$. Terahertz spectroscopy has been employed as a powerful technique for exploring the high-frequency electronic and far-infrared optical properties of a wide range of materials including liquids, gases, and crystals [99-101]. Terahertz radiation is highly sensitive to low-energy intraband excitation of free carriers and lattice vibrations; hence, fundamental dynamics of these excitations can be explored [102]. Also, using pulsed THz radiation, quasi-particles such as free carriers, phonons, and polarons can be characterized and distinguished since they have distinct signatures in the terahertz $(\mathrm{THz})$ range $[103,104]$. Moreover, it is possible to study non-equilibrium carrier dynamics and AC photoconductivity with sub-picosecond time resolution [105-107].

Until recently frequencies that fall within the THz range were referred to as a "THz gap" due to the technical difficulties associated with the development of strong (and/or coherent) sources and detectors at these frequencies. However, in recent decades, this technological gap has diminished as new technologies and applications have emerged rapidly. THz spectroscopy has found wide applications in material characterization [108], security [109], medical imaging [110], communication technology [111], and quality control of food [108].

A recent $\mathrm{THz}$ revolution began in late 1980 with the improvement of free-space propagation of $\mathrm{THz}$ pulses generated and detected by photoconductive switching [112]. At about the same time optical rectification (OR) was experimentally demonstrated by Bass et al [113]. In 1982 Valdmanis et al. built the first electro-optic sampling system with picosecond resolution [114]. In 1992, THz generation from OR in a nonlinear crystal was demonstrated by Zhang et al [115-117]. A few years later, THz detection by electro-optic sampling in nonlinear crystals was discovered [118,119]. After that, OR has been widely used to generate THz pulses for $\mathrm{THz}$ time-domain spectroscopy (THz-TDS) and time-resolved $\mathrm{THz}$ spectroscopy (TRTS). Difference-frequency generation [120], photoconductive switching [121], and four-wave mixing in optically generated plasma [122] are other methods of generating THz pulses which are derived from femtosecond (fs) optical pulses. 


\subsection{The Wave Propagation}

The propagation of $\mathrm{THz}$ waves and their interaction with matter can be understand using classical electromagnetic theory. Let us consider the macroscopic Maxwell equations in the presence of matter to describe $\mathrm{THz}$ waves.

$$
\begin{gathered}
\nabla \cdot \vec{D}=\rho_{f}, \\
\nabla \cdot \vec{B}=0, \\
\nabla \times \vec{E}=-\frac{\partial \vec{B}}{\partial t}, \\
\nabla \times \vec{H}=\overrightarrow{J_{f}}+\frac{\partial \vec{D}}{\partial t},
\end{gathered}
$$

where $\vec{E}$ and $\vec{B}$ are the electric field and the magnetic field. $\rho_{f}$ and $\overrightarrow{J_{f}}$ are the free charge density and current density in the medium. The electric displacement field $\vec{D}$ and the magnetizing field $\vec{H}$ can be described using $\vec{E}$ and $\vec{B}$ as

$$
\begin{gathered}
\vec{D} \equiv \epsilon_{o} \vec{E}+\vec{P}=\varepsilon(1+\chi) \vec{E}=\varepsilon \vec{E}, \\
\vec{H} \equiv \frac{1}{\mu_{o}} \vec{B}-\vec{M}=\frac{1}{\mu} \vec{B},
\end{gathered}
$$

where $\varepsilon_{o}$ and $\mu_{o}$ are the permittivity and the permeability of free space respectively. $\varepsilon$ and $\mu$ are the permittivity and the permeability of the matter respectively. Polarization $\vec{P}$ and magnetization $\vec{M}$ provide information about the macroscopic electromagnetic properties of the medium. It can be assumed that $\overrightarrow{f_{f}}$ is linear with the electric field.

$$
\vec{J}_{f}=\sigma \vec{E}
$$

where $\sigma$ is the electric conductivity of the medium. In the absence of free charge $\left(\rho_{f}=0\right)$, the wave equation can be expressed by taking the curl of equation 2.3 and using the relations in equation (2.5) and (2.6) as follows 


$$
\left(\nabla^{2}-\frac{1}{c^{2}} \frac{\partial^{2}}{\partial t^{2}}\right) E=\mu_{0}\left(\frac{\partial J}{\partial t}-\frac{\partial^{2} P}{\partial t^{2}}\right)
$$

where $c\left(=1 / \sqrt{\epsilon_{0} \mu_{0}}\right)$ is the speed of light. Equation 2.8 implies that the time-varying polarization $P$ and conduction current $J$ serve as sources and will give rise to electromagnetic radiation. These source terms describe different processes of $\mathrm{THz}$ generation. The polarization term describes $\mathrm{THz}$ generation by difference-frequency generation and optical rectification which will be described further in section 2.3. The current term describes $\mathrm{THz}$ emission from photoconductive switches and air plasma generation. In a non-conducting, linear dielectric medium, the wave equation can be simplified as

$$
\nabla^{2} \vec{E}=\frac{1}{v^{2}} \frac{\partial^{2} \vec{E}}{\partial t^{2}}
$$

where $v=c / \tilde{n}$ is the phase velocity of the wave inside the medium and $\tilde{n}$ is the refractive index. The general solution to equation 2.9 is a linearly polarized monochromatic plane wave with angular frequency $\omega$ propagating in $z$ direction through a dielectric medium.

$$
E(z, t)=E_{0} e^{i(k z-\omega t)},
$$

where $k=\omega \tilde{n} / c$. The complex refractive index provides information about the optical properties of the medium. It can be expressed as

$$
\tilde{n}(\omega)=n(\omega)+i \kappa(\omega)=n(\omega)+i \frac{\alpha(\omega) c}{2 \omega}
$$

where $\kappa(\omega)$ is the extinction coefficient and $\alpha$ is the absorption coefficient, which describes the absorption losses when propagating through the medium. Substituting the complex refractive index in equation 2.10 yields

$$
E(z, t)=E_{0} e^{i \omega(n z / c-t)} e^{-\alpha z / c}
$$

If the wave is propagating through the dielectric medium with thickness $d$, there will be a phase shift due to refraction and attenuation due to absorption. 
Then, the plane wave can be expressed in the frequency domain as

$$
E(\omega)=E_{0}(\omega) e^{-\frac{\alpha(\omega) d}{2}} e^{-i \frac{n(\omega) d}{c} \omega} .
$$

\subsection{THz sources and optical Rectification}

When electromagnetic radiation is incident on a material, bound electrons in the material will be displaced, resulting a polarization. For sufficiently weak applied field $\tilde{E}(t)$, bound charges reside in a perfectly parabolic potential and experiences a linear restoring force. In this linear optical regime, the induced polarization $\tilde{P}(t)$ of the medium linearly depend on the applied electric field as follows

$$
\tilde{P}(t)=\varepsilon_{o} \chi^{(1)} \tilde{E}(t)
$$

where $\chi^{(1)}$ is the linear susceptibility tensor and $\varepsilon_{o}$ is the permittivity of free space. Hence, if the input electric field has is sinusoidal the polarization will follow that sine wave. However, if the system is driven by a strong electric field, bound charge will experience a non-parabolic potential and response nonlinearly to the electric field. In this nonlinear regime, the dependence of polarization on the electric field can be expressed as a power series $[123,124]$

$$
\begin{aligned}
\tilde{P}(t)=\varepsilon_{o}\left[\chi^{(1)} \tilde{E}(t)\right. & \left.+\chi^{(2)} \tilde{E}^{2}(t)+\chi^{(3)} \tilde{E}^{3}(t)+\cdots\right] \\
& \equiv \tilde{P}^{(1)}(t)+\tilde{P}^{(2)}(t)+\tilde{P}^{(3)}(t),
\end{aligned}
$$

where $\chi^{(2)}$ and $\chi^{(3)}$ are second and third-order non-linear optical susceptibility tensors. Hence, if the input of the electric field is a sinusoid with a strong amplitude the polarization will follow that sine wave and develop new sine waves with different frequencies.

Figure 2.1(a) shows the linear response of polarization to a low electric field and figure 2.1(b) shows the nonlinear polarization response to a high electric field. Figure 2.1(c) shows a toy model for an asymmetric potential energy with a quadratic term (dashed line) and a cubic term (dashed-dotted line). The solid thick line shows the total potential energy. The electron motion shows a nonlinear response. Figure 2.1 (d) shows the linear ( $x_{L}$, dashed line) and nonlinear $\left(x_{N L}\right.$, dashed-dotted line) motion of electrons. Figure 2.1 (e) shows Fourier components of the secondorder polarization: a DC offset (OR) and second harmonic generation (SHG) [102]. 
For small electric fields, linear polarization oscillates with the same frequency as the driving field and second-order effects are negligible. For high electric fields, polarization has a strong second-order nonlinear contribution and linear contribution. Second-order nonlinear optical interactions occur only in noncentrosymmetric crystals while third-order nonlinear interactions occur in both centrosymmetric and noncentrosymmetric crystals at sufficiently high field amplitudes
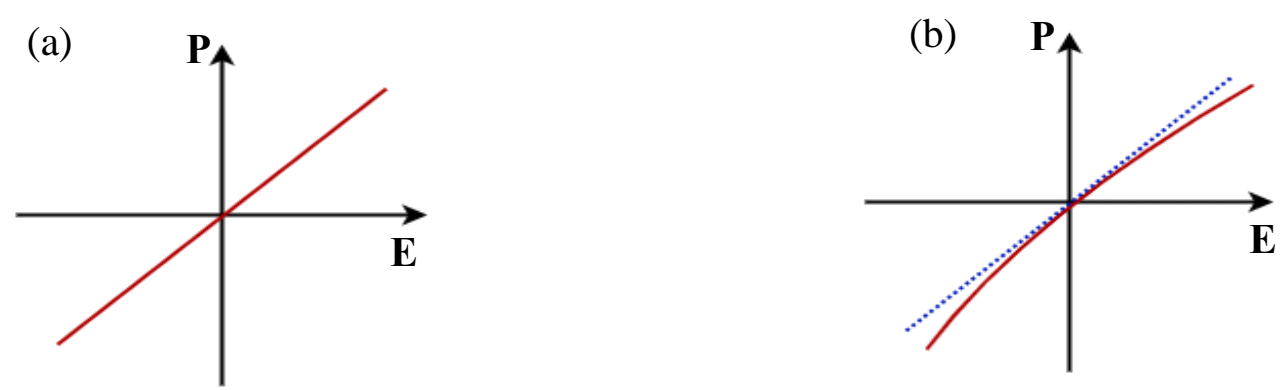

(c) $U(x)=\frac{1}{2} m \omega_{0} x^{2}+\frac{1}{3} m \alpha x^{3}$
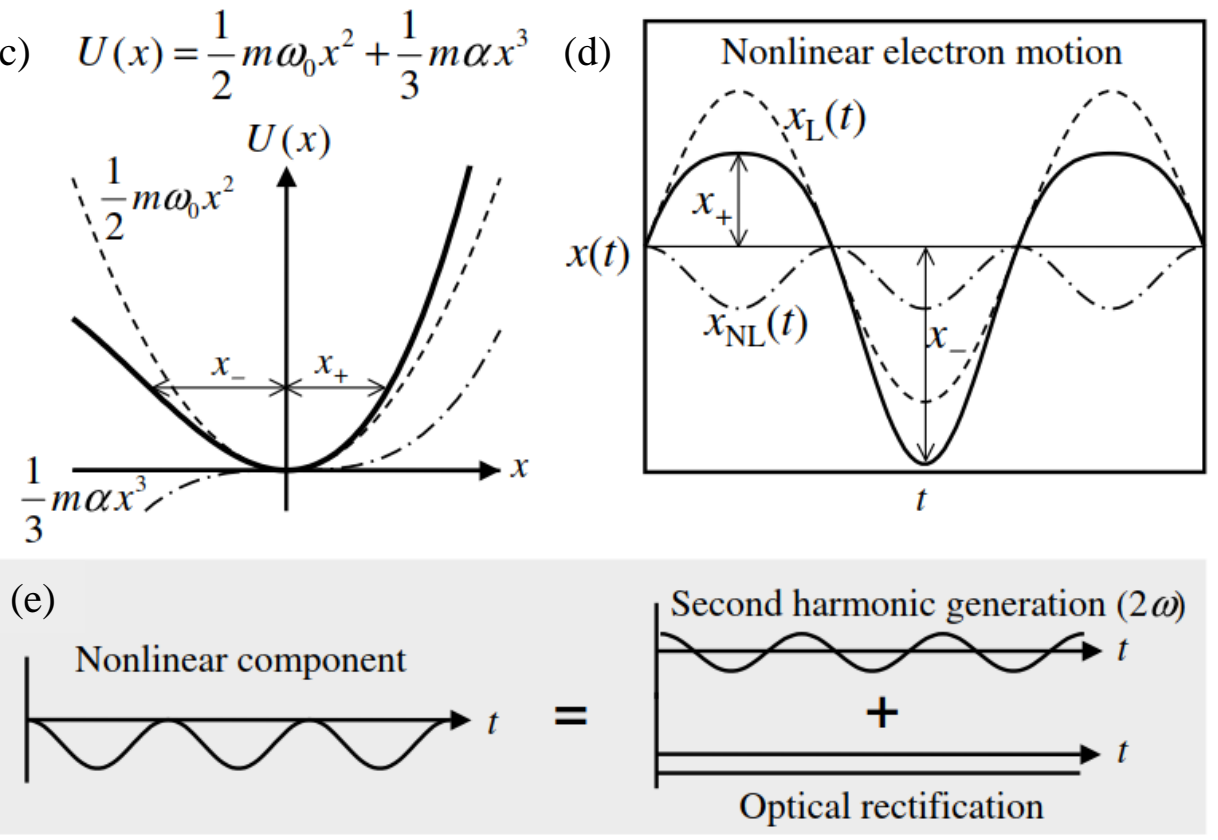

Figure 2.1 (a) linear response of polarization on the applied field. (b) Nonlinear response of polarization. (c) Model electric potential energy. (d) Nonlinear response of the electron motion. (e) Two second-order nonlinear optical processes: OR and SHG. The figures (c)-(e) taken from ref [102] 
Let us consider the wave equation for nonlinear optical media, which is given as

$$
\nabla^{2} \tilde{E}-\frac{1}{c^{2}} \frac{\partial^{2} \tilde{E}}{\partial t^{2}}=\frac{1}{\epsilon_{o} c^{2}} \frac{\partial^{2} \tilde{P}}{\partial t^{2}}
$$

This equation implies that the time-varying polarization $\tilde{P}(t)$ can act as the source of electromagnetic radiation. The source term containing $\mathrm{P}$ describes the different nonlinear process as follows [123]

$$
\begin{gathered}
\tilde{P}^{(2)}(t)=\epsilon_{o} \chi^{(2)}\left[E_{1}^{2} e^{-2 i \omega_{1} t}+E_{2}^{2} e^{-2 i \omega_{2} t}+2 E_{1} E_{2} e^{-i\left(\omega_{1}+\omega_{2}\right) t}+2 E_{1} E_{2}^{*} e^{-i\left(\omega_{1}-\omega_{2}\right) t}+c . c\right] \\
+2 \epsilon_{o} \chi^{(2)}\left[E_{1} E_{1}^{*}+E_{2} E_{2}^{*}\right] .
\end{gathered}
$$

The complex amplitudes of the various components of the second-order polarization are given by

$$
\begin{array}{ll}
\left.\begin{array}{l}
P\left(2 \omega_{1}\right)=\epsilon_{o} \chi^{(2)} E_{1}^{2} e^{-2 i \omega_{1} t} \\
P\left(2 \omega_{2}\right)=\epsilon_{o} \chi^{(2)} E_{2}^{2} e^{-2 i \omega_{1} t}
\end{array}\right\} & \text { Second harmonic generation (SHG) } \\
P\left(\omega_{1}+\omega_{2}\right)=2 \epsilon_{o} \chi^{(2)} E_{1} E_{2} e^{-i\left(\omega_{1}+\omega_{2}\right) t} & \text { Sum of frequency generation (SFG) } \\
P\left(\omega_{1}+\omega_{2}\right)=2 \epsilon_{o} \chi^{(2)} E_{1} E_{2} e^{-i\left(\omega_{1}-\omega_{2}\right) t} & \text { Difference frequency generation (DFG) } \\
P(0)=2 \epsilon_{o} \chi^{(2)}\left[E_{1} E_{1}^{*}+E_{2} E_{2}^{*}\right] & \text { Optical rectification (OR). }
\end{array}
$$

(a)

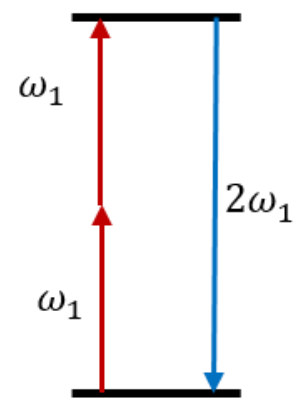

(b)

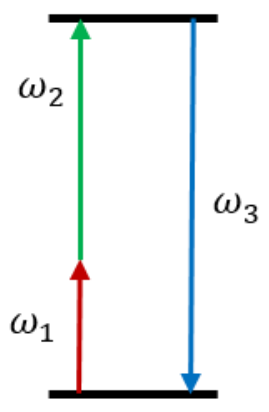

(c)

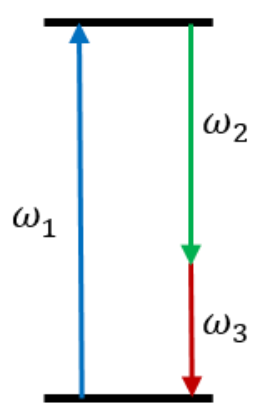

(d)

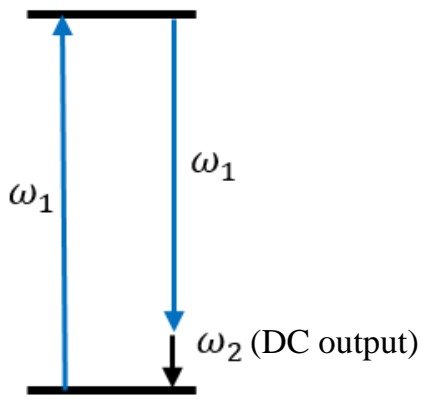

Figure 2.2. Energy level diagram of (a) second-harmonic generation, (b) sum of frequency generation, (c) difference-frequency generation and (d) optical rectification.

Figure 2.2 shows the energy level diagram of various second-order nonlinear processes. In SHG, two photons with the same frequency interact with a nonlinear media to generate photons 
with twice the frequency of the initial photons. SFG is equivalent to the SHG except that two input photons have different frequencies. Here, two photons with a frequency $\omega_{1}$ and $\omega_{2}$ generate new photon with a frequency $\omega_{1}+\omega_{2}$. In DFG, the frequency of the output photon is the difference between two incident photons. It can be noted that equation 2.18 consists of a zero-frequency term this will result in OR in which a static electric field is created.

As indicated by equation (2.18), rectified polarization induced by continuous waves will not radiate. However, if the electromagnetic wave is time-dependent, e.g in pulsed lasers, the induced polarization is also time-varying and results in electromagnetic radiation. Since the spectral bandwidth of the radiation is the inverse of the optical pulse duration, broadband $\mathrm{THz}$ pulses can be generated by OR of femtosecond pulses in a nonlinear medium as shown in figure 2.3. OR is one of the most commonly used methods for generating ultrashort $\mathrm{THz}$ pulses. OR only occurs in non-centrosymmetric crystals. However, it is possible to generate THz pulses by OR in centrosymmetric crystals, if the crystal symmetry is reduced, either at the surface or by applying external electrical or mechanical forces.

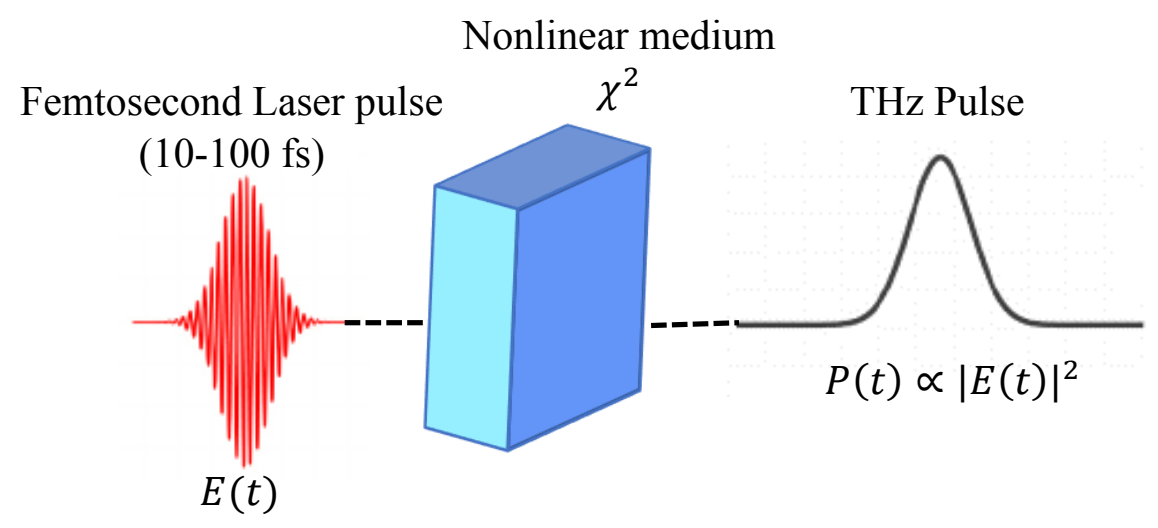

Figure 2.3. Nonlinear polarization induced by optical rectification

\subsection{Recording the complex spectrum}

The common methods used to detect the THz electric field are electro-optic (EO) and photoconductive sampling. In this work, EO sampling is used to detect the $\mathrm{THz}$ radiation. EO sampling - based on the Pockels effect - is a second-order nonlinear optical process. A low 
frequency or static electric field induces birefringence for an electromagnetic wave at the optical frequencies propagating through the nonlinear medium. If the optical field is a short pulse of duration much shorter than the THz pulse, time of flight of the two pulses can be set such that the THz electric field appears to the optical field as a quasi-DC electric field in a sufficiently thin EO crystal. Then the electromagnetic wave will experience the birefringence induced by the static field. The relative time of flight of each pulse can be varied so that the optical field probes, or samples, different parts of the THz pulse and the entire THz pulse "transient" can be mapped out.

Let us consider the second-order nonlinear polarization of electro-optic effect, given as [102]

$$
P_{i}^{(2)}(\omega)=2 \sum_{j, k} \epsilon_{0} \chi_{i j k}^{(2)}(\omega, \omega, 0) E_{j}(\omega) E_{k}(0)
$$

where $i, j$ and $k$ are indices for cartesian components of each field and $\chi_{i j k}^{(2)}$ is the relevant non-zero second-order nonlinear susceptibility tensors. In a lossless medium, $\chi_{i j k}^{(2)}(0, \omega,-\omega)=$ $\chi_{i j k}^{(2)}(\omega, \omega, 0)$, and thus equation 2.19 can be expressed as follows

$$
P_{i}^{(2)}(\omega)=2 \sum_{j, k} \epsilon_{0} \chi_{i j k}^{(2)}(0, \omega,-\omega) E_{j}(\omega) E_{k}(0)
$$

By comparing equation 2.20 with second-order nonlinear polarization of OR, $P_{i}^{(2)}(0)=\sum_{j, k} \epsilon_{0} \chi_{i j k}^{(2)}(0, \omega,-\omega) E_{j}(\omega) E_{k}^{*}(\omega)$, it can be seen that the OR and the EO effect have the same nonlinear coefficient. These can be related through the electro-optic coefficient $R$.

Figure 2.4 shows the setup of $\mathrm{THz}$ detection by free-space electro-optic sampling and polarization scheme that allows for its detection. A linearly-polarized probe pulse passes through the $\mathrm{EO}$ crystals. Without $\mathrm{THz}$ field, the probe (or gate) pulse will not experience any birefringence. Thus, linear polarization of the probe pulse will not change after passing through the EO crystal. A $\lambda / 4$-wave plate converts the linearly polarized probe pulse into a circularly polarized pulse. Then a Wollaston prism splits the probe pulse into two orthogonal linearly polarized components which have equal irradiance which are measured on balanced photodetectors. The presence of the $\mathrm{THz}$ field induces a birefringence in the EO crystal, which produces a slightly elliptical 
polarization of the probe pulse. After the $\lambda / 4$ wave plate, the probe polarization develops into almost circular, but still elliptical polarization. The Wollaston prism split the probe beam into two imbalanced orthogonal components and two balanced detectors measure the intensity difference between these two orthogonal components.

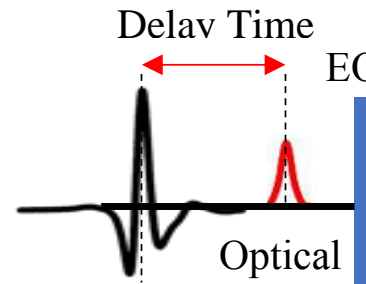

THz pulse pulse

\section{Initial}

Polarization
After EO

Crystal

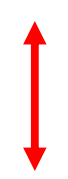

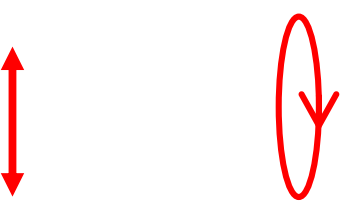

Balanced Detectors

Without THz Field

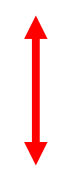

With THz Field

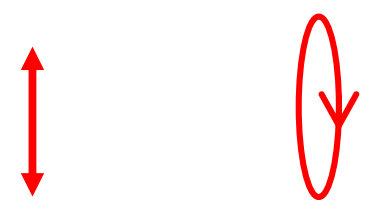

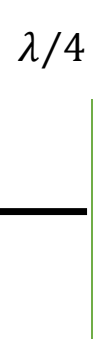

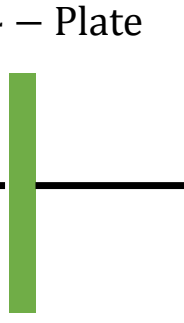

After $\lambda / 4$ Plate
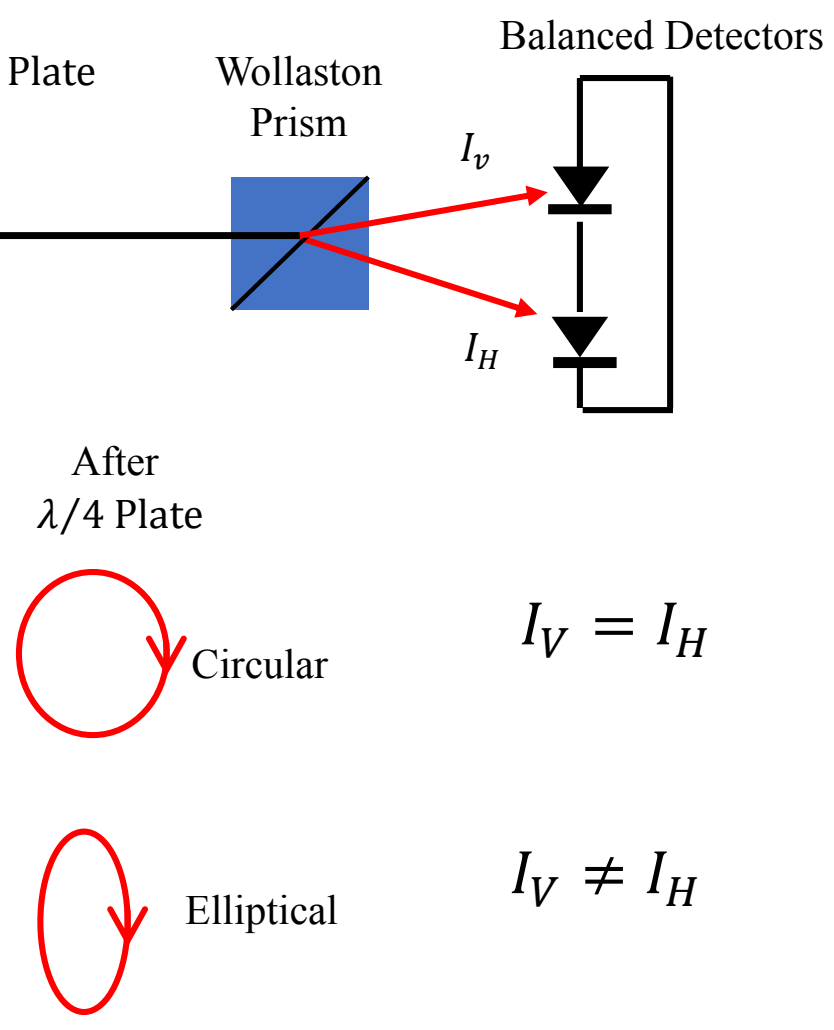

$I_{V} \neq I_{H}$

Figure 2.4. Typical Electro-optic sampling setup. Polarization of the optical pulse with and without $\mathrm{THz}$ field after polarization optics [102].

Commonly use crystals for $\mathrm{THz}$ detection are zinc telluride ( $\mathrm{ZnTe}$ ), gallium phosphide $(\mathrm{GaP})$ and gallium selenide (GaSe). The linear EO coefficients are listed in table 2.1. In this thesis, $\mathrm{ZnTe}$ is used as the detection crystal. Figure 2.5 shows the polarization of the probe beam and $\mathrm{THz}$ field in a standard EO-sampling setup. The maximum EO signal can be obtained when both optical beam polarization and THz electric field are parallel to the [1]0] axis of an $\langle 110\rangle$ oriented crystal. 


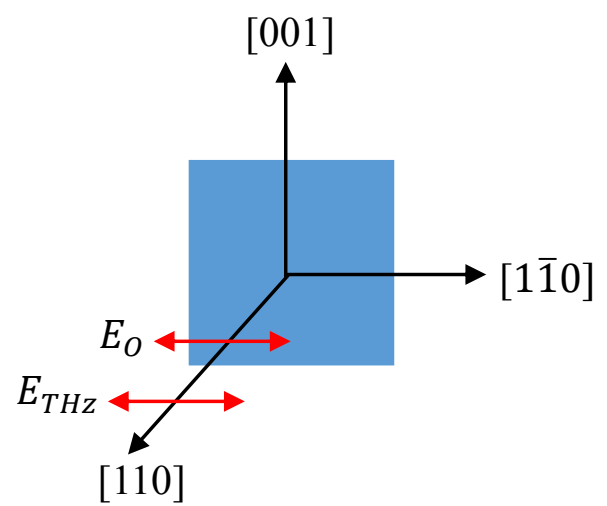

Figure 2.5. The crystal axis of a $\mathrm{ZnTe}$ crystal and the polarization of $\mathrm{THz}$ field $\left(E_{T H z}\right)$ and optical beam $\left(E_{o}\right)$.

The differential phase retardation $\nabla \phi$ experienced by the optical probe pulse is given as [102]

$$
\nabla \phi=\left(n_{x}-n_{y}\right) \frac{\omega L}{c}=\frac{\omega L}{c} n_{O}^{3} r_{41} E_{T H z}
$$

where $L$ is the crystal length, $\omega$ is the probe frequency, $n_{O}$ is the refractive index at the optical frequency and $r_{41}$ is the electro-optic coefficient. The irradiances of the two orthogonal components are given as

$$
\begin{aligned}
& I_{x}=\frac{I_{0}}{2}(1-\sin \Delta \phi) \approx \frac{I_{o}}{2}(1-\Delta \phi), \\
& I_{y}=\frac{I_{0}}{2}(1+\sin \Delta \phi) \approx \frac{I_{o}}{2}(1+\Delta \phi),
\end{aligned}
$$

where $I_{0}$ is the irradiance of the probe beam. Thus, the irradiance difference $\Delta I$ between two orthogonal components is

$$
\Delta I(\Delta t)=I_{o} \Delta \phi=I_{o} \frac{\omega}{c} n_{o}^{3} r_{41} L \propto E_{T H z}(\Delta t) .
$$

It can be seen that $\Delta I$ is proportional to the THz electric field experienced by the probe pulse. Thus, by varying the delay time $\Delta t$ between $\mathrm{THz}$ pulse and probe pulse the $\mathrm{THz}$ transient can be recorded. 


\subsection{Nonlinear crystals for Terahertz generation}

To determine the efficiency of the $\mathrm{THz}$ generation by $\mathrm{OR}$, it is crucial to understand the tensor formalism of the susceptibility. The second-order nonlinear susceptibility tensor $\chi_{i j k}^{(2)}$ can be expressed in a contacted notation: $d_{i l}=\chi_{i j k}^{(2)} / 2$. Using this contacted notation, equation 2.18 can be expressed as

$$
\left(\begin{array}{l}
P_{x} \\
P_{y} \\
P_{z}
\end{array}\right)=2 \epsilon_{0}\left(\begin{array}{llllll}
d_{11} & d_{12} & d_{13} & d_{14} & d_{15} & d_{16} \\
d_{21} & d_{22} & d_{23} & d_{24} & d_{25} & d_{26} \\
d_{31} & d_{32} & d_{33} & d_{34} & d_{35} & d_{36}
\end{array}\right)\left(\begin{array}{c}
E_{x}^{2} \\
E_{y}^{2} \\
E_{z}^{2} \\
2 E_{y} E_{z} \\
2 E_{z} E_{x} \\
2 E_{x} E_{y}
\end{array}\right)
$$

For highly symmetric crystals, most tensor elements are zero and only a few elements are nonzero. For the zincblende crystal structures with $\overline{4} 3 m$ point group symmetry $d_{14}, d_{25}$ and $d_{36}$ are the only nonzero elements and all these elements are equal. For chalcopyrite crystal structure with $\overline{4} 2 \mathrm{~m}$ point group symmetry, nonzero elements are $d_{14}=d_{25}$ and $d_{36}$. Here $d_{14}$ and $d_{25}$ values are similar through Kleinman symmetry [123].

Binary (II-VI and III-V) semiconductors, such as zinc telluride (ZnTe), gallium phosphide (GaP) and gallium selenide (GaAs), and wide-gap semiconducting oxides, such as lithium niobate $\left(\mathrm{LiNbO}_{3}\right)$ and lithium tantalate $\left(\mathrm{LiTaO}_{3}\right)$, are nonlinear crystals that can be used for $\mathrm{THz}$ generation by OR [125]. Recently chalcopyrite crystals, such as CSP, ZGP, CGP, and AgGaS 2 , have demonstrated good $\mathrm{THz}$ generation because of their comparable or higher nonlinearity $[47,126]$. Figure 2.6 shows the $d_{36}$ nonlinear optical coefficients as a function of band gap and effective primitive cell size of II-IV-V $\mathrm{V}_{2}$ chalcopyrites and GaAs for comparison. The data for figure 2.6 were taken from Ref. [127,128]. It is seen that $d_{36}$ decreases with increasing band gap and increases with increasing effective primitive cell size $\left(a_{e f f}\right)$.

The efficiency of the $\mathrm{THz}$ generation and detection is determined by a material's linear electro-optical (EO) coefficient, $\boldsymbol{r}_{i j k}$; see Table 2.1 for a list of uniaxial crystals - namely crystals with a single axis of rotational symmetry. The relationship between second-order nonlinear susceptibility and EO coefficient is $\chi_{i j k}=-\left(n^{4} / 2\right) r_{i j k}$, where $n$ is the material's linear refractive 
index. To achieve efficient $\mathrm{THz}$ generation by OR, crystals should have a large EO coefficient, high nonlinearity, suitable thickness, and suitable crystal orientation with respect to the linear polarization of the pump radiation.

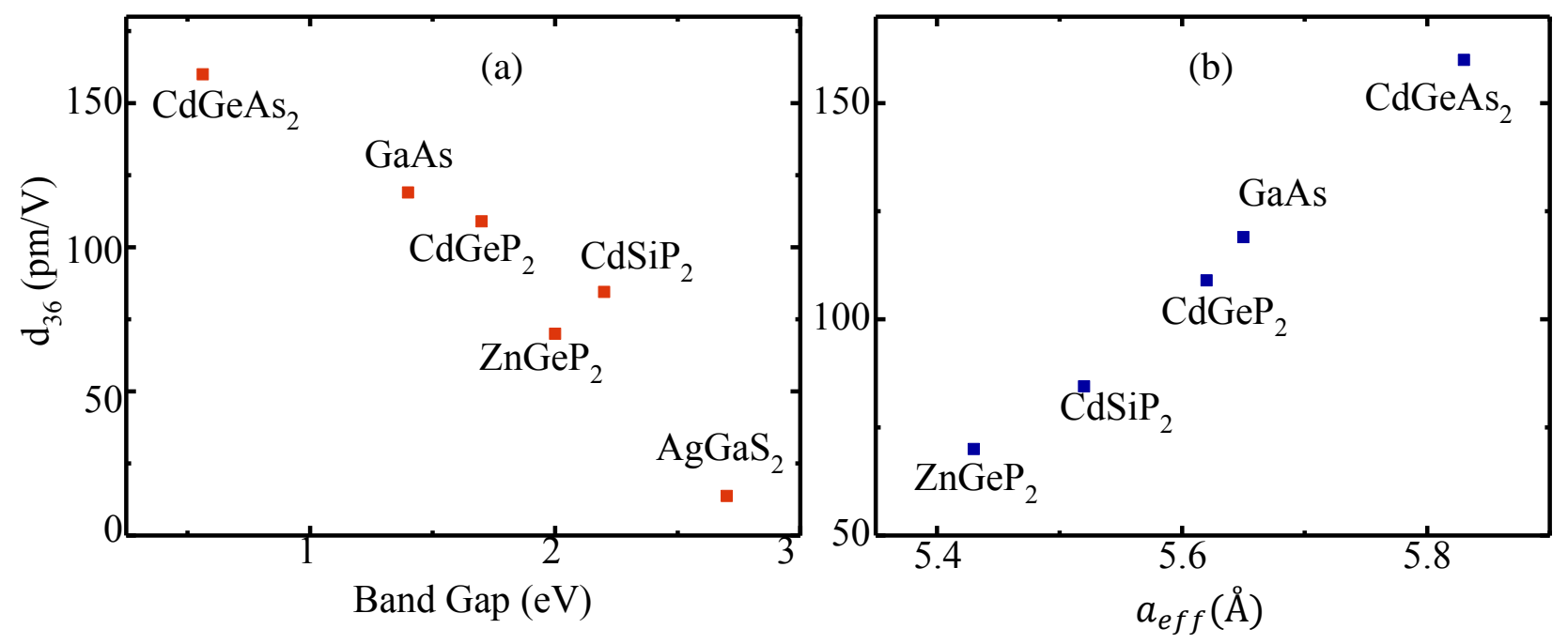

Figure 2.6. Nonlinear coefficient $d_{36}$ of II-IV- $V_{2}$ chalcopyrites and GaAs for comparison as a function of (a) band gap (b) effective primitive cell size.

Coherence length and optical phonon modes in the crystal determine the bandwidth of an EO crystal. The coherence length measures the degree of velocity matching. It is defined as the distance at which the phase difference between optical pulse and THz wave is equal to $\pi / 2$. The coherence length is given as [129]

$$
l_{c}=\frac{c}{2 v_{T H z}\left|n_{g r}-n_{T H z}\right|},
$$

where $c$ is the speed of light, $n_{T H z}$ is the refractive index at the THz frequencies $v_{T H z}$ and $n_{g r}=$ $n_{O}(\lambda)-\lambda \frac{\partial n_{o}}{\partial \lambda}$ is the group refractive index of the optical wavelength $\lambda$ and corresponding refractive index $n_{O}$. The crystal thickness should be less or equal to the coherence length.

Absorption in the terahertz region also limits the bandwidth of $\mathrm{THz}$ generation in a nonlinear crystal. Transverse-optical (TO) phonon resonances results in strong $\mathrm{THz}$ absorption in EO crystals. 
Table 2.1. electro-optic coefficient and transparency range of selected nonlinear crystals.

\begin{tabular}{|c|c|c|}
\hline Material & $\begin{array}{c}\text { Transparency } \\
\text { Range }(\boldsymbol{\mu m})\end{array}$ & $\boldsymbol{r}_{41}(\boldsymbol{p m} / \boldsymbol{V})$ \\
\hline $\mathrm{ZnTe}$ & $0.55-25[130]$ & $4.0[131]$ \\
\hline $\mathrm{GaP}$ & $0.54-10.5[132]$ & $0.97[125]$ \\
\hline $\mathrm{GaAs}$ & $0.9-17.3[130]$ & $1.5[132]$ \\
\hline $\mathrm{GaSe}$ & $0.62-20[133]$ & $14.4[134]$ \\
\hline $\mathrm{CdGeP}_{2}$ & $0.7-14[135]$ & $3.0[135]$ \\
\hline $\mathrm{ZnGeP}_{2}$ & $0.74-15[130]$ & $1.6[136]$ \\
\hline $\mathrm{CdSiP}_{2}$ & $0.5-9[137]$ & - \\
\hline
\end{tabular}

To characterize the THz generation in CGP, ZGP, and CSP chalcopyrite crystals, a pump irradiance ("intensity") dependence is performed. Figure 2.7 shows the amplitude of the $\mathrm{THz}$ electric field amplitude, $\left|E_{p p}\right|$, as a function pump intensity for several pump photon energies below the optical bandgap of each crystal. All graphs are plotted over the same range. The inset of figure 2.7 (c) shows the THz emission over an extended range at $1.55 \mathrm{eV}$. CGP sample shows the strongest $\mathrm{THz}$ emission at $0.805 \mathrm{eV}$. Among all three crystals, CSP sample has the strongest emission for the other two excitation energies.

At low pump intensities, $\mathrm{E}_{\mathrm{THz}}$ increases linearly with excitation density because $d E_{T H z} / d z \propto d_{36} I(z)$, where $I$ is the pump intensity and $\mathrm{z}$ is the distance of pulse propagation within the crystal. Here effective tensor element $d_{\text {eff }}$ and $d_{36}$ are interchangeable since all crystals have same cut. At higher intensities, THz emission saturates due to nonlinear absorption. Linear absorption and nonlinear absorption can be modeled by a generalized Beer's law as follows

$$
\frac{d I(z)}{d z}=-\alpha I(z)-\beta I^{2}(z)-\gamma I^{3}(z)
$$

Here $\alpha$ is the usual linear absorption coefficient, $\beta$ is the two-photon absorption (2PA) coefficient and $\gamma$ is the three-photon absorption (3PA) coefficient. 

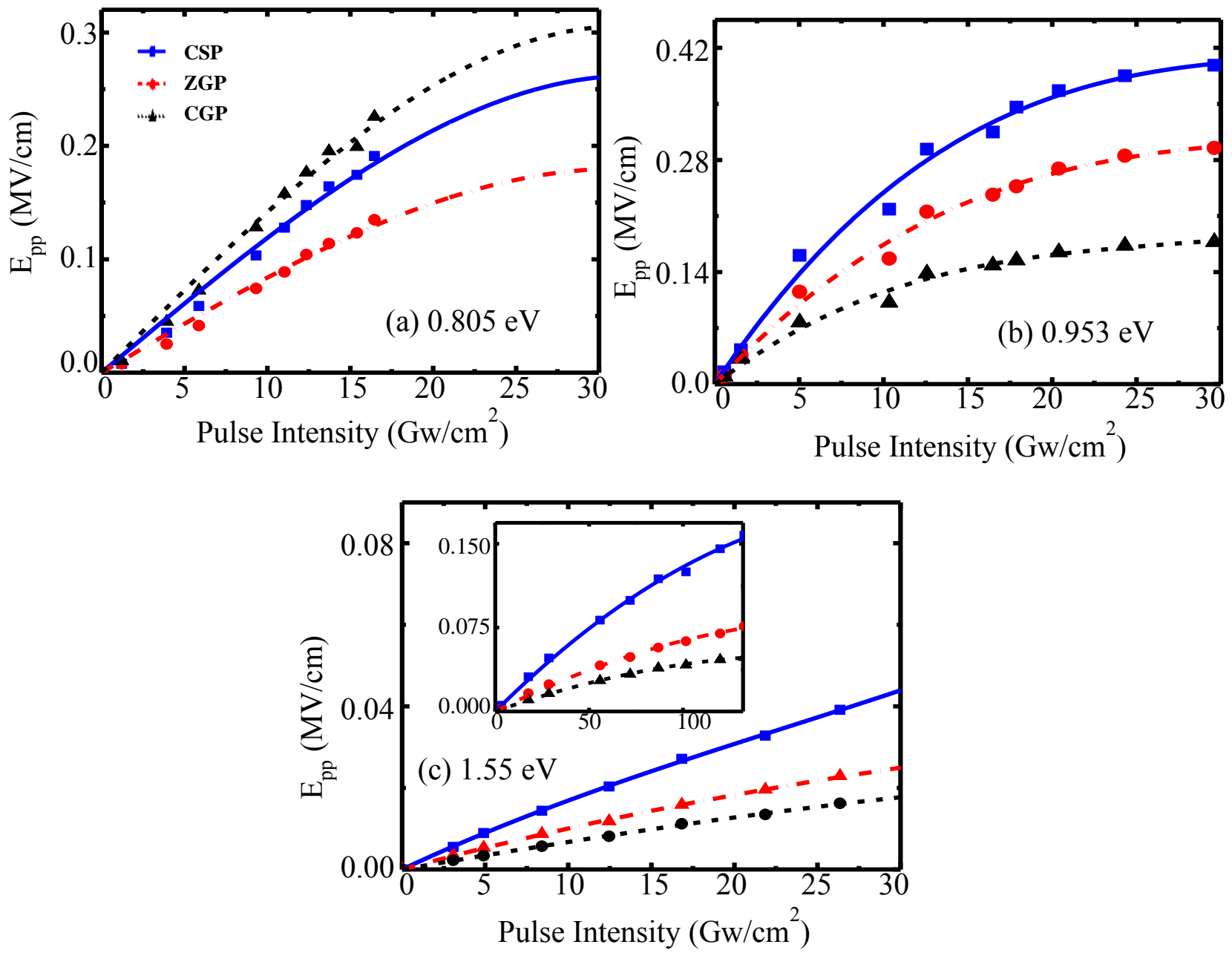

Figure 2.7. Amplitude of the THz electric field as a function of pulse intensity for CGP, ZGP and CSP, excited at (a) $0.805 \mathrm{eV}$, (b) $0.953 \mathrm{eV}$ and (c) $1.55 \mathrm{eV}$. The inset of (c) shows an extended range. Figure modified from Piyathilaka et al. [47]

Values used to fit the data are shown in table 2.2. At $0.805 \mathrm{eV}$, absorption is dominated by 3PA for all crystals. Absorption is dominated by $2 \mathrm{PA}$ for all three crystals at $1.55 \mathrm{eV}$. For ZGP and CSP absorption is dominated by 3PA whereas, for ZGP, absorption is dominated by $2 \mathrm{PA}$. 
Table 2.2. Fit values for the absorption of integrated $\mathrm{THz}$ emission

\begin{tabular}{cccc}
\hline & $\propto\left(\mathrm{cm}^{-1}\right)$ & $\beta\left(\mathrm{cm}^{-1} \mathrm{GW}\right)$ & $\gamma\left(\mathrm{cm}^{-1} \mathrm{GW} W^{2}\right)$ \\
\hline CGP & 14.52 & $(0.805 \mathrm{eV})$ & \\
ZGP & 15.87 & - & 0.0004 \\
CSP & 11.73 & - & 0.0001 \\
& & - & 0.0002 \\
CGP & 15.83 & $(0.953 \mathrm{eV})$ & \\
ZGP & 17.01 & 0.2047 & 0.0045 \\
CSP & 12.33 & - & 0.0091 \\
\hline CGP & 31.18 & - & - \\
ZGP & 17.88 & 0.0015 & - \\
CSP & 16.96 & 0.0019 & - \\
\hline
\end{tabular}

\subsection{Experimental Setup}

THz experiments are performed using a Ti:sapphire-based chirped-pulse amplifier (CPA), which is operating with a central wavelength of $800 \mathrm{~nm}$ and $1-\mathrm{kHz}$ repetition rate to produce 100fs pulses with average beam power $>3.1$ Watts. The output of the laser amplifier is split into two replicas - one to generate THz by OR and another to measure it by EO sampling as shown in figure 2.8. OR occurs by weakly focusing a laser pulse into 0.5-mm-thick, (110)-cut CSP source crystal at normal incidence with the optical polarization oriented azimuthally along the [ $\overline{1} 10]$ axis to maximize the generation. A high-density polyethylene low-pass filter transmits the $\mathrm{THz}$ radiation, which is collected and focused onto a 0.3-mm-thick, (110)-cut ZnTe EO-sampling crystal using two off-axis parabolic mirrors (OAPMs). The second laser pulse probes the THz pulse creating an EO signal as a function of delay time and is deciphered by a Soleil-Babinet compensator, Wollaston polarizing prism, and two balanced photodiodes (A, B) that record the THz electric field with a lock-in amplifier. The lock-in-amplifier is referenced to the modulation of the $\mathrm{THz}$ generation beam, modulated by a mechanical chopper prior to the OR crystal. All the optical 
components from $\mathrm{THz}$ source to detector are enclosed in a chamber and purged with dry nitrogen/air to reduce absorption due to water vapor.

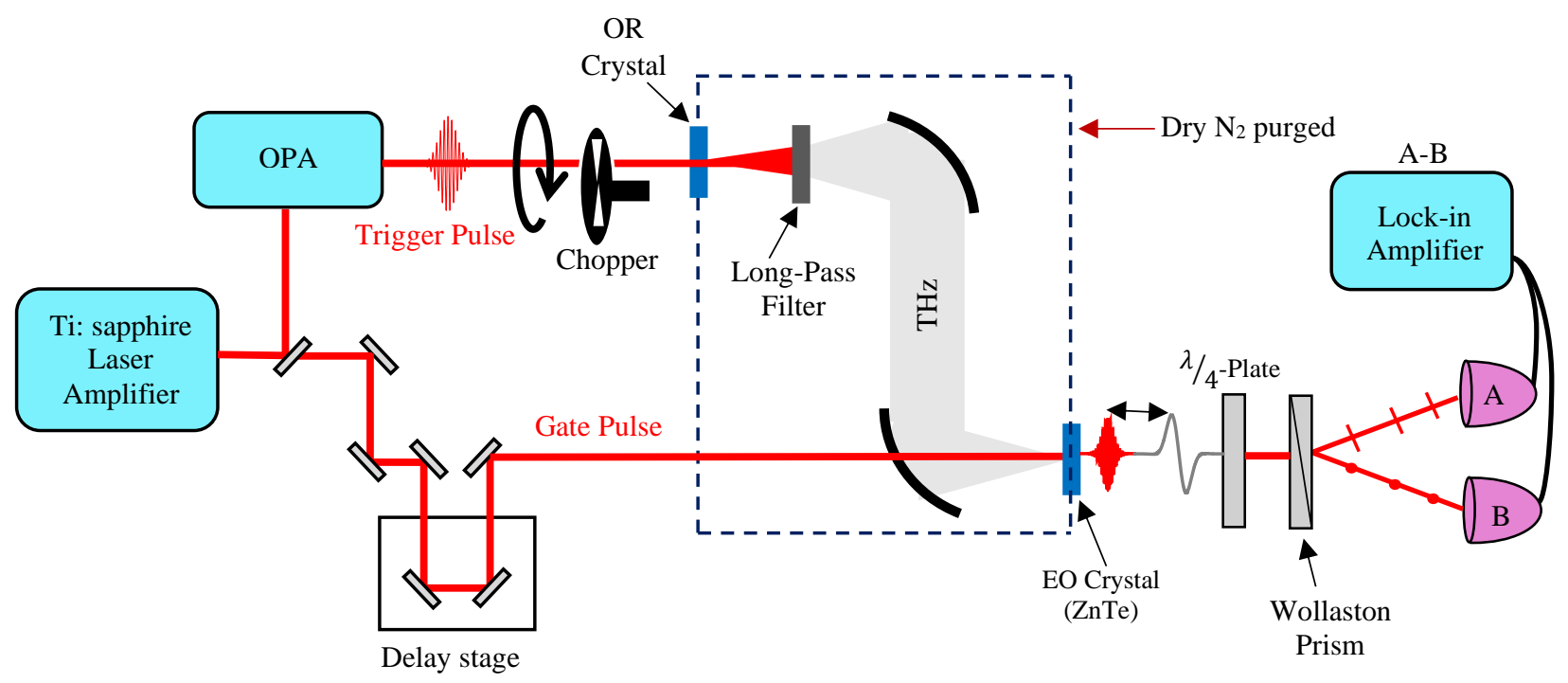

Figure 2.8. Schematic diagram of a typical $\mathrm{THz}$ experiment setup.

Figure 2.9 shows the typical emission transients from a CSP crystal pump at $800 \mathrm{~nm}$ and detected by EO sampling in the $0.3 \mathrm{~mm}$ thick ZnTe crystal. A primary peak is observed around zero delay time and is the signal that is used for most analysis in this thesis. The strength of the $\mathrm{THz}$ pulse can be characterized in numerous ways, but a simple method is to take the peak-to-peak electric field amplitude $E_{p p}$ as illustrated. A replica of the THz signal at $11 \mathrm{ps}$ results from the reflection of the optical probe pulse in the detection crystal resampling the THz pulse. Water has many absorption lines due to the molecular rotational transitions in the vibrational ground or excited state in the $\mathrm{THz}$ band. As shown in figure 2.9 (a) these ambient absorptions introduce additional wiggles in the transient. To reduce these water absorptions, all-optical components from THz source to detector in the experimental setup are enclosed in a chamber and purged with dry nitrogen/dry air. Figure 2.9 (b) shows that by purging with dry nitrogen or dry air additional wiggles in the transient can be reduced. 


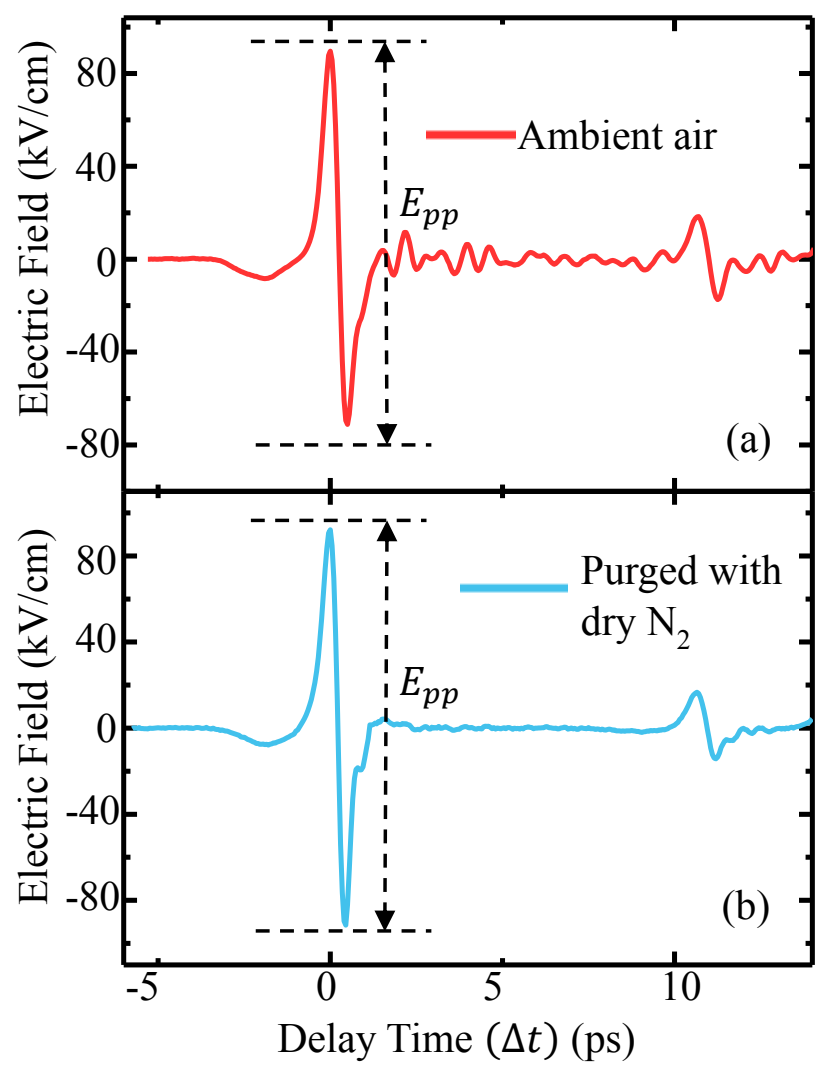

Figure 2.9. Temporal waveform of a THz pulse generated by optical rectification in $0.5 \mathrm{~mm}$ thick (110) CSP crystal. (a). Without purged (b). Purged with dry nitrogen. The replica of THz signal at $11 \mathrm{ps}$ is due to internal reflection in the detection crystal

Fourier transform (FT) can be applied to transform a real-valued time-domain signal into a complex-valued frequency-domain spectrum [138]

$$
E(t) \stackrel{F T}{\rightarrow} \frac{1}{\sqrt{2 \pi}} \int_{-\infty}^{\infty} E(t) e^{-i \omega t} d t=E(\omega) .
$$

Fast Fourier transform (FFT) is a type of Fourier transform and is commonly used in signal processing. FFT is an algorithm-based technique that reduces the number of computations needed. Figure 2.10 shows the FFT of the time-domain spectra (a) ambient air (b) purged with dry nitrogen. It can be seen that the terahertz electric field in ambient air leads to absorption dips at $1.2 \mathrm{THz}, 1.4$ $\mathrm{THz}$, and $1.7 \mathrm{THz}$ compared to the $\mathrm{THz}$ electric field in a nitrogen atmosphere. 


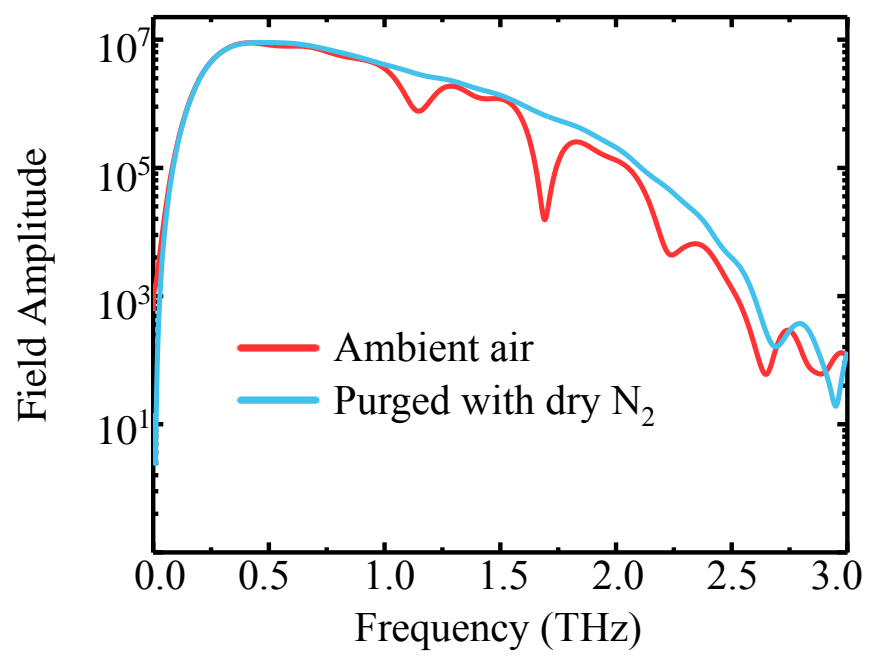

Figure 2.10. THz electric field in the presence of water vapor (red curve) shows absorption dips compared to $\mathrm{THz}$ field propagating in a nitrogen atmosphere (blue curve).

\subsection{Terahertz Time-Domain Spectroscopy (THz-TDS)}

THz-TDS has emerged as a powerful tool in investigating optical and electrical properties of materials in the far-infrared region of the electromagnetic spectrum. Since THz photons have energies on the order of a few $\mathrm{meV}$, it is sensitive to the response of free electrons and lattice vibrations in solids. It is also sensitive to the rotational transitions in molecules, vibrational motions and even magnetic resonance. Hence using THz-TDS fundamental dynamics of these excitations can be explored.

The major aspect of THz-TDS is it allows to probe the free-carrier conductivity of the sample. Traditional contact-based Hall-effect measurements are commonly used to investigate electrical transport properties. However, it is challenging to measure accurate conductivity and mobility using Hall-effect measurements since it is difficult to make near-zero resistance contacts. Accurate measurements require compensating for the junction created by the contacts and can only be performed in expendable samples. Major advantages of THz-TDS compared to Hall effect measurements are: It is a non-contact, hence non-invasive, technique and it measures carrier dynamics over picoseconds to nanoseconds time scale [139]. One advantage of this method is that the electric field of the $\mathrm{THz}$ pulses can be measured directly, such that a Fourier transform from the time domain gives a complex response spectrum. Thus, the material or devices can be analyzed 
through the refractive index, extinction coefficient, dielectric function and AC conductivity without performing Kramers-Kronig analysis [99,140,141]. Carrier density and scattering time of the sample can be extracted by fitting conductivity spectra with an appropriate conduction model.

Since the electric field is detected as a function of time in THz-TDS, it is termed as timedomain spectroscopy. THz-TDS typically performed as a transmission measurement. In this technique the amplitude and phase of incident $\mathrm{THz}$ electric field, $E_{\text {sam }}(\mathrm{t})$, and transmitted electric field, $E_{\text {ref }}(\mathrm{t})$, through the sample are obtained. The complex transmission coefficient, $T(\omega)$, is obtained from the Fourier transform of the THz electric field through the sample, $E_{s a m}(\omega)$, and free space, $E_{\text {ref }}(\omega)$. Terahertz transmission coefficient is directly related to the ground state conductivity. This will be further discussed in chapter 3 .

To perform THz-TDS, the experimental setup shown in figure 2.8 was modified by adding a second set of OAPMs, creating an intermediate focus where a sample can be located - as shown in figure 2.11. To perform temperature-dependent measurements, the sample is situated in a cryostat which can controllably vary the lattice temperature in the range $4 \mathrm{~K}-300 \mathrm{~K}$. Figure 2.12 (a) shows the time-domain spectrum through CSP crystal and free space at $300 \mathrm{~K}$. The transmitted $\mathrm{THz}$ pulse experiences absorption and delay due to its interaction with the sample. For each transient, the lock-in time constant was set to $0.3 \mathrm{~s}$ and the at least 3 scans were averaged. The averaged transients are treated with an arctan window function to remove the first reflection of the gate pulse in ZnTe EO crystal. Prior to performing a numerical Fourier transform, the transients were extended by zero-padding to the end of the 400 (measured) data points to give 16,384 (total) data points. Then Fourier transform is performed on the experimental transients. Figure 2.12 (b) shows the corresponding amplitude of the numerical Fourier transform of the time-domain spectra. Decrease in amplitude spectra of CSP sample compared to the free space spectra is due to the absorption. 


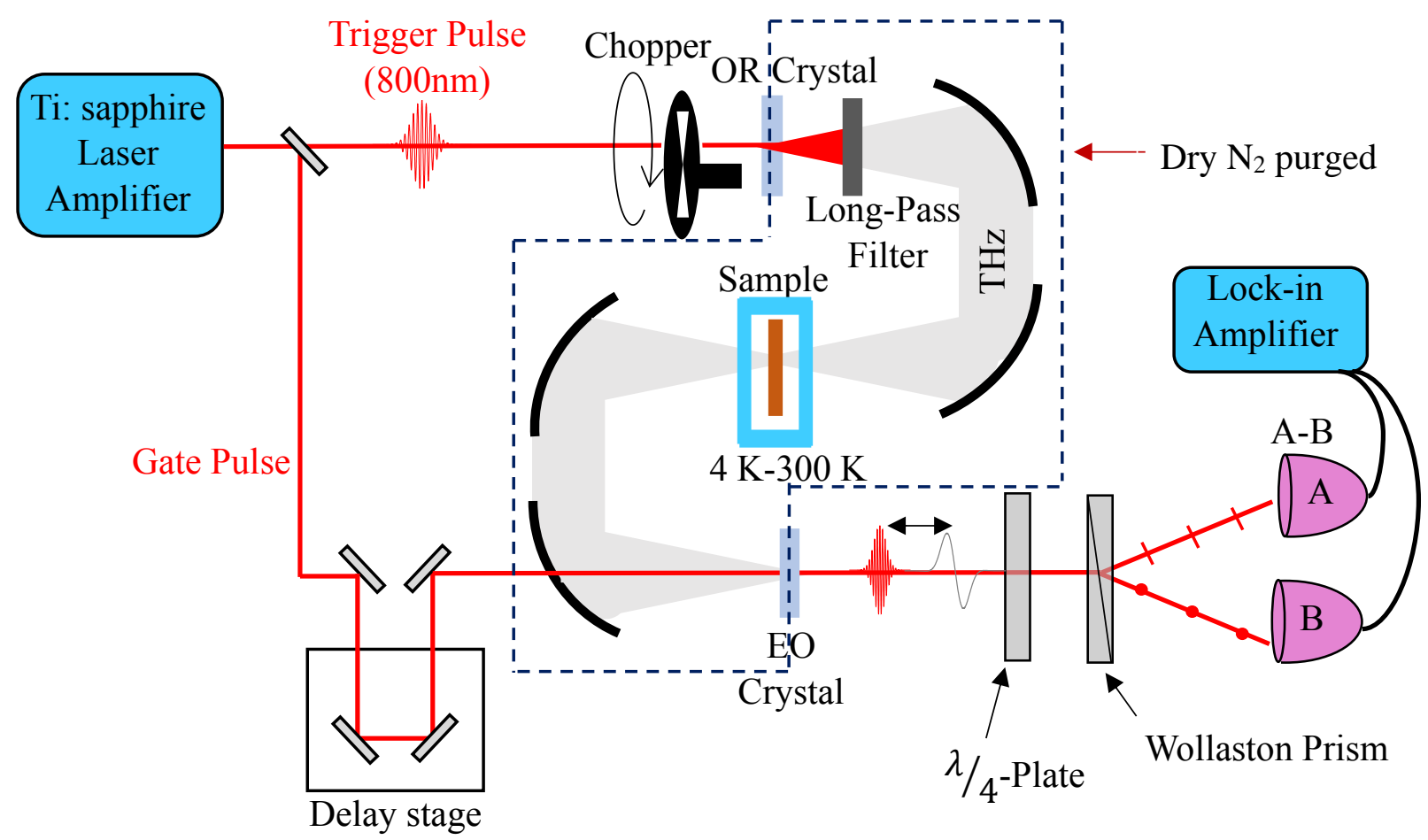

Figure 2.11. Schematic diagram of a typical THz-TDS system
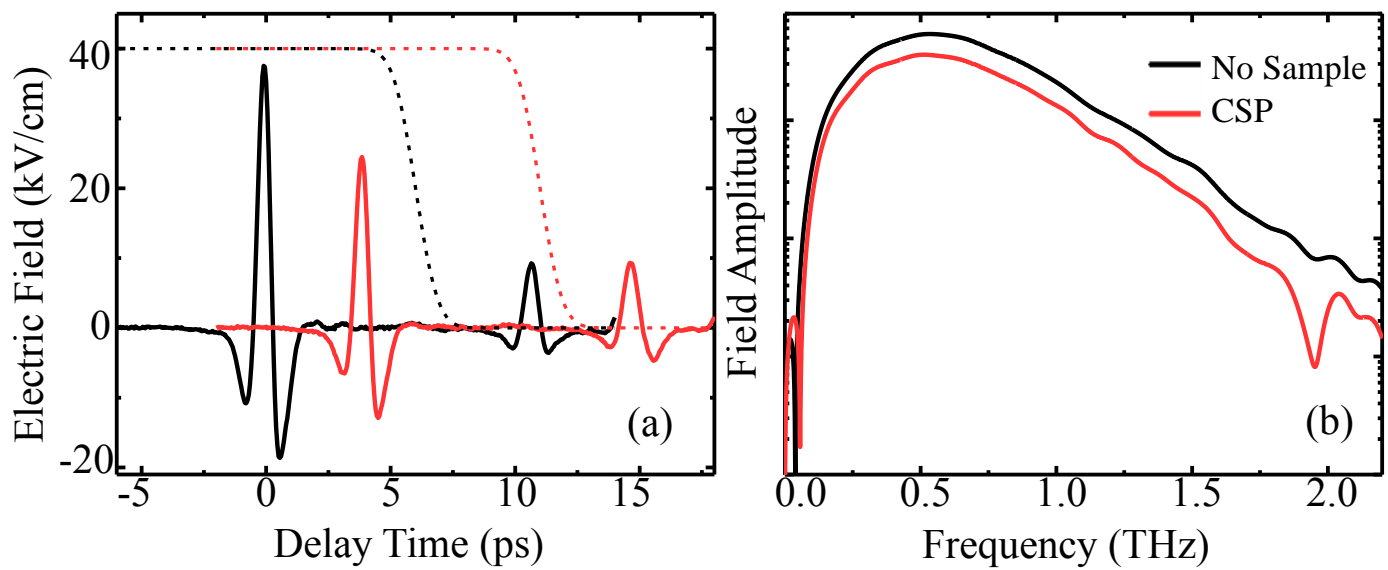

Figure 2.12. (a) Typical time-domain transient of terahertz propagating through free space (between source and detector) or transmitted through $\mathrm{CdSiP}_{2}$ at $300 \mathrm{~K}$.(b) Corresponding amplitude spectra in the frequency domain. 


\subsection{Time-Resolved Terahertz Spectroscopy (TRTS)}

TRTS is a non-contact probe of photoexcited charge-carrier mobility and the complex dispersion of $\mathrm{THz}$ frequency light due to photoexcited carriers resulting from an optical pump pulse. TRTS can determine non-equilibrium carrier dynamics and the transient electrodynamic response of materials with a temporal resolution of better than $200 \mathrm{fs}$ in a non-invasive manner (assuming the pump pulse has a shorter pulse width than the THz probe pulse) $[105,139,142]$. The electronic response is referred to as the AC photoconductivity. Other ultrafast techniques, such as time-resolved luminescence and transient-absorption spectroscopy are sensitive to either the sum or the product of the electron and hole population density and do not measure their conductivity directly. In particular, TRTS is ideal to probe electrical conduction of nanostructured materials because it does not require contact and attaching of wires to such small sample [106], although measuring ensembles of nanostructured materials may require analysis of scattering, mean-field, and percolation effects [143].

In a typical TRTS experiment, carriers are excited with an optical pump that allows one to probe the time-dependent conductivity using transient $\Delta E(t)$. To perform TRTS, THz-TDS experimental setups are modified by adding an optical beam with a variable delay time using another mechanical translation stage. Figure 2.13 shows the modified experimental setup for TRTS. The pump beam is mechanically chopped by a chopper and the modulation frequency is once again used for reference in the electronic detection apparatus. Here, the delay time between optical pump and THz probe is controlled by Delay Stage 2 .

For $\mathrm{AC}$ photoconductivity measurements, fractional changes in transmission $\Delta E(\omega) / E_{0}$ is recorded at the peak of the THz pulse. This measures how $\Delta E / E_{0}$ changes as a function of time after optical injection of photocarriers. Delay stage 1 is fixed at the peak of the THz pulse and pump Delay Stage 2 is varied. In this way, it is feasible to characterize the average far-IR response and investigate the decay times and recombination mechanisms [105]. For AC photoconductivity measurements, $\Delta E / E_{0}$ may be recorded as a function of the optical pump frequency (pump photon energy) at a fixed time after optical injection. Delay Stage 2 is fixed, and position of the Delay Stage 1 is varied to record the entire $\mathrm{THz}$ waveform from which the complex $\mathrm{AC}$ photoconductivity spectrum can be obtained [105]. 


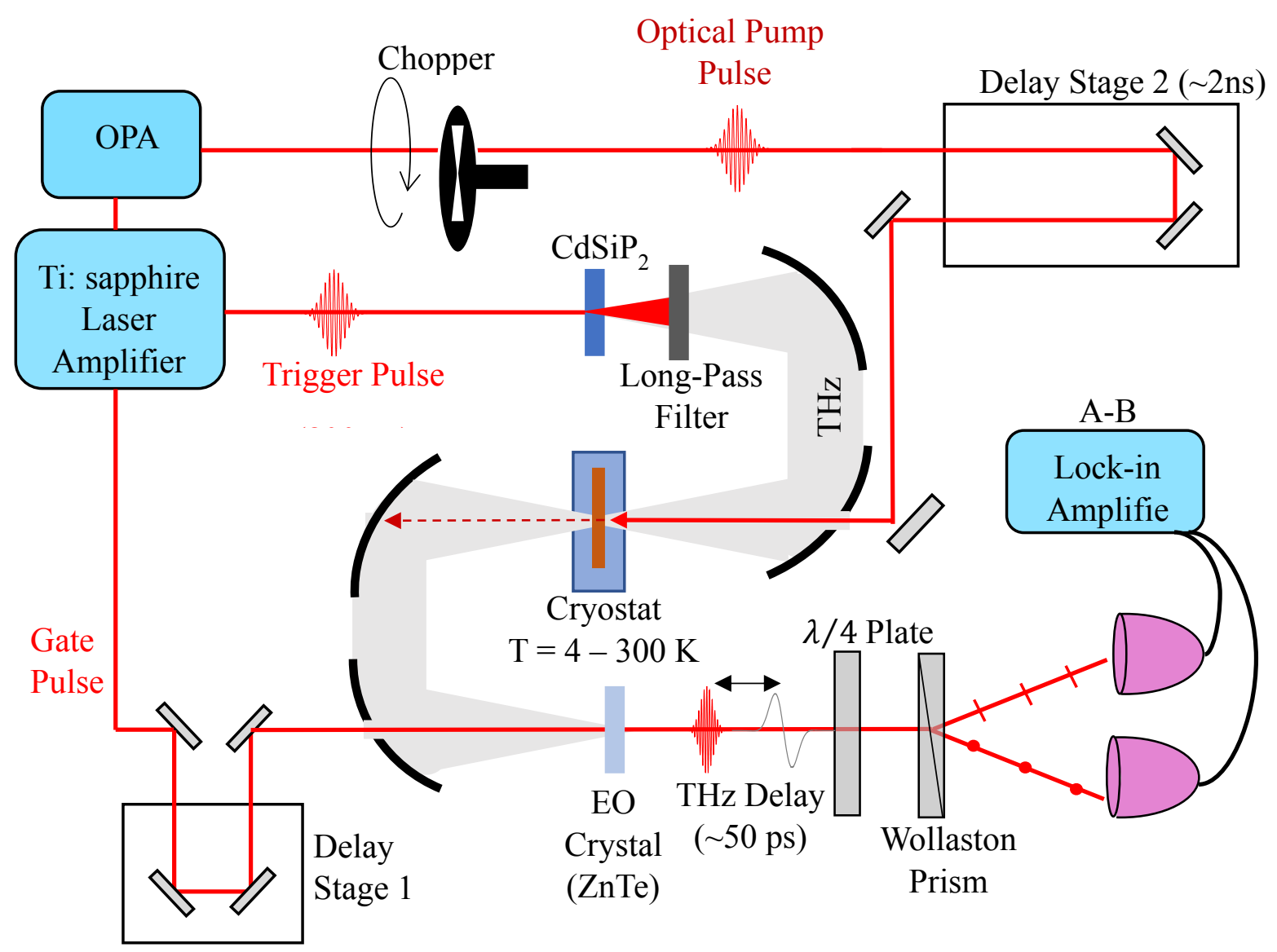

Figure 2.13.Schematic diagram of the TRTS experimental setup. The sample is placed at the intermediate $\mathrm{THz}$ focus and is photoexcited by an above-band gap optical pulse. Delay Stage 2 is used to control the delay time between optical pump and terahertz probe.

\subsection{Transient-Absorption Spectroscopy}

Ultrafast carrier processes in materials take place on a timescale of femtoseconds to hundreds of picoseconds. Using conventional electronic detection methods, these ultrafast dynamics cannot be temporally resolved since the response time of the conventional detection methods are limited to about one nanosecond. Transient-absorption spectroscopy (or pump-probe) spectroscopy can be used to investigate the dynamic processes that occur within very short periods. Pump-probe spectroscopy is a powerful technique for characterizing structural and electronic properties of excited states of materials [144,145]. In this technique, a strong spectrally narrow pump pulse with a pulse width on the order of $100 \mathrm{fs}$ is used to excite electrons into nonequilibrium 
states. A second weaker, spectrally broad probe pulse measures the perturbations caused by pump pulse [146]. By sending the probe pulse through a delay stage, delay time, $\tau$, between the pump and probe is varied. Then normalized differential absorption is $\Delta A(\tau) / A_{0}=\left[A(\tau)-A_{0}\right] / A_{0}$, where the differential absorption, $A(\tau)-A_{0}$, is measured naturally by the lock-in amplifier in the presence of the pump pulse and $A_{0}$ is the absorption of the un-excited (ground state) of the system, measured without the presence of the pump pulse. Changing the delay time, $\tau$, maps out the transient response. Similar to TRTS, $\Delta A(\tau) / A_{0}$ measures the excited-state carrier population, and its relaxation and recombination dynamics. Control of the excitation condition (optical frequency, irradiance and polarization with respect to the probe pulse) provide access to a wide range of excited-state charge-carrier-related phenomena.

Bleach

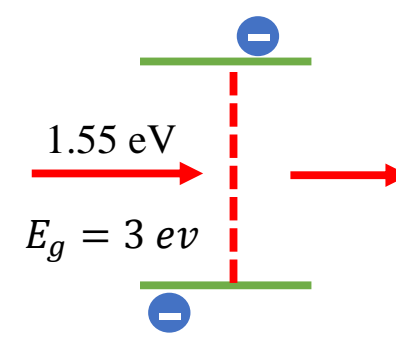

Ground State

Absorption

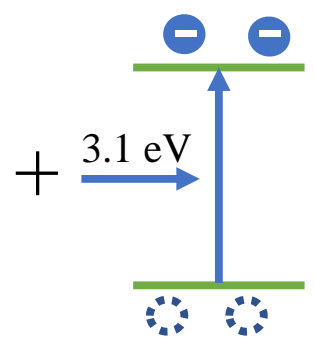

Pump

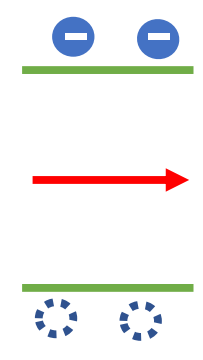

Excited State

No Absorption

Absorption

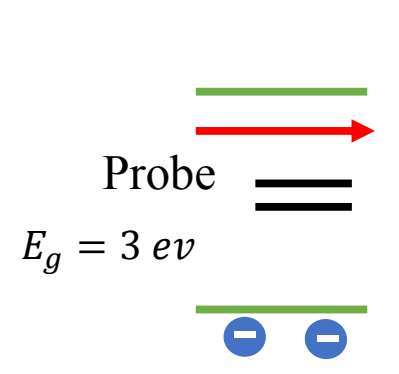

Ground State

No Absorption

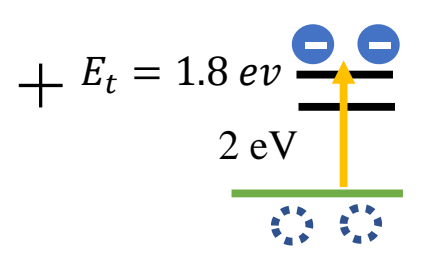

Pump

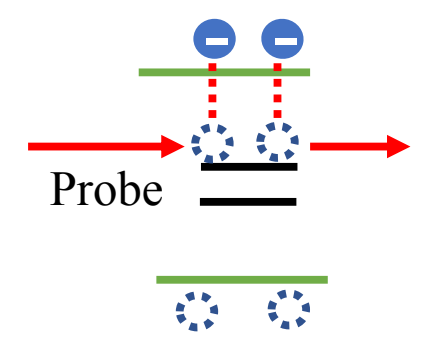

Excited State

Absorption

Figure 2.14. Schematic diagram of ground-state bleaching and excited-state absorption. 
Figure 2.14 shows some of the processes that can contribute to the transient-absorption signal. A "bleach" occurs if excited carriers reduce the overall optical absorption of the probe (or increases the probe transmission). Conversely, "absorption" occurs when excited carriers increase the probe absorption (reduces the probe transmission). The former case can occur when photoexcited carriers deplete the available electrons from the ground state, such that the probe can no longer make those transitions [147]. In direct-gap semiconductors, this is similar to the static process of a doping-induced Burstein-Moss shift of the band-gap with respect to the intrinsic value. The latter case can occur when excited-carriers allow more available transitions for the probe pulse, as is often the case when selecting the pump energy to promote carriers to or from shallow or mid-gap dopant state in semiconductors and is responsible for dynamics that are attributed to Shockley-Reed-Hall (SRH) processes. Alternatively, free-carrier absorption of electrons in the conduction band or holes in the valence band can also lead to an absorption signal (not shown in the figure), and their dynamics can be differentiated from SRH processes due to the pump irradiance scaling of their signal strength and the short-lived decay times.

\subsubsection{Experimental Setup}

Figure 2.15 shows the schematic diagram of the pump-probe experimental setup used in this work. The pump-probe experimental is performed using a Ti:sapphire laser amplifier operating at a central wavelength of $800 \mathrm{~nm}, 1-\mathrm{kHz}$ repetition rate, an average power of $>3.0 \mathrm{~W}$ and $100-\mathrm{fs}$ pulse duration. The output of the laser amplifier is split into two replicas - one to excite the sample

and another to probe the sample. The pump pulses are passing through a $\beta$-barium borate (BBO) crystal to frequency-double the pulse to $400 \mathrm{~nm}$ central wavelength. Then 400 -nm pulses are passed through a short-pass filter to blocks residual $800-\mathrm{nm}$ pulses. A mechanical chopper at a reference frequency $0.25 \mathrm{kHz}$ modulates the pump pulse and is synchronized to the laser-amplifier repetition rate. The delay time between pump and probe pulses is varied by a mechanical translational stage that the equivalent temporal range of $\sim 3 \mathrm{~ns}$. The pump and probe pulses are loosely focused to a $1 / e^{2}$ spot diameter of $290 \mu \mathrm{m}$ and $90 \mu \mathrm{m}$ respectively. Probe pulses (at a center wavelength of $800 \mathrm{~nm}$ ) are sent to a Si photodetector which is designed to detect light ranging from $350 \mathrm{~nm}$ to $1100 \mathrm{~nm}$ with peak performance at $970 \mathrm{~nm}$. It has a peak response of 
$0.65 \mathrm{~A} / \mathrm{W}$ and diode capacitance $60 \mathrm{pF}$. The signal is recorded with a lock-in amplifier. The entire system is computer-controlled.

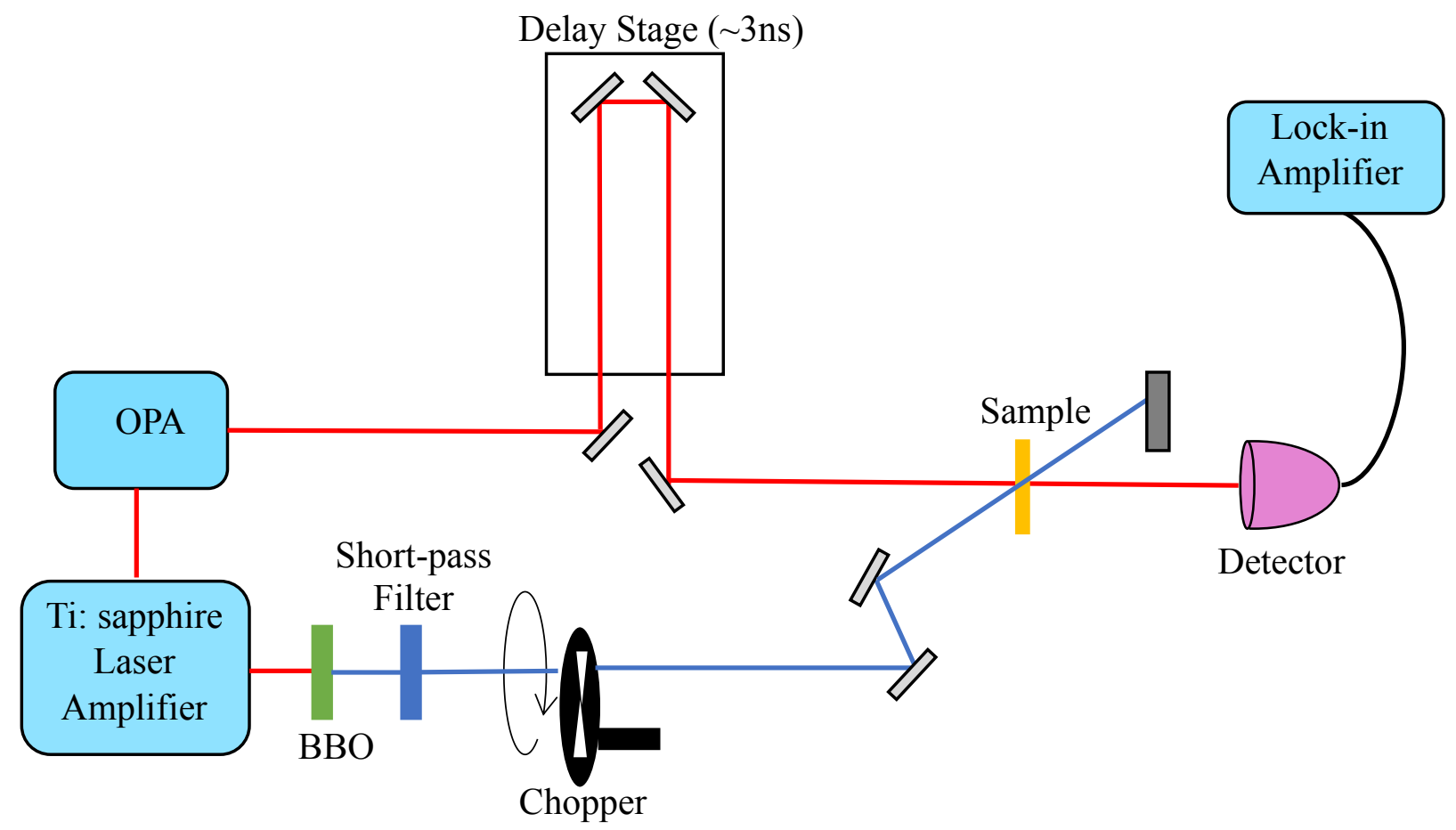

Figure 2.15. Schematic diagram of the ultrafast pump-probe setup

\subsection{Samples}

Chalcopyrite crystals are ternary compounds with $\mathrm{ABC}_{2}$ chemical formula. There are two types of chalcopyrite semiconductors: I-III-V1 2 and II-IV-V 2 compounds which belongs to the $I \overline{4} 2 d$ space group and $\overline{4} 2 m$ point group symmetry. Figure 2.16 shows the derivation of the chalcopyrite structure from the diamond structure. The binary compounds III-V and II-VI can be derived from the group IV class of tetrahedrally bonded semiconductors according to the GrimmSommerteld rule [33]. This states that there must be an average of four valance atoms per atomic site. This can be extended to predict other groups of materials like I-III-V1 $1_{2}$ and II-IV-V 2 compounds. 


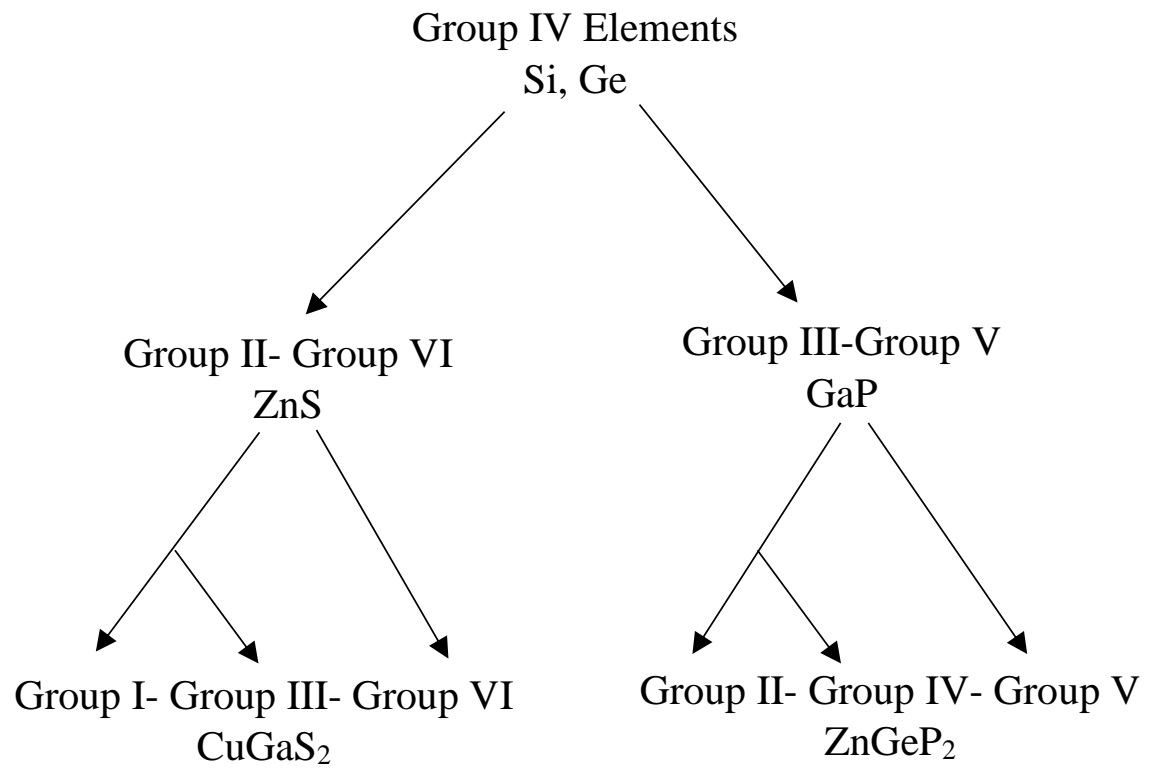

Figure 2.16. Origin of I-III- $-\mathrm{VI}_{2}$ and II-IV-V 2 chalcopyrite structure from the diamond structure

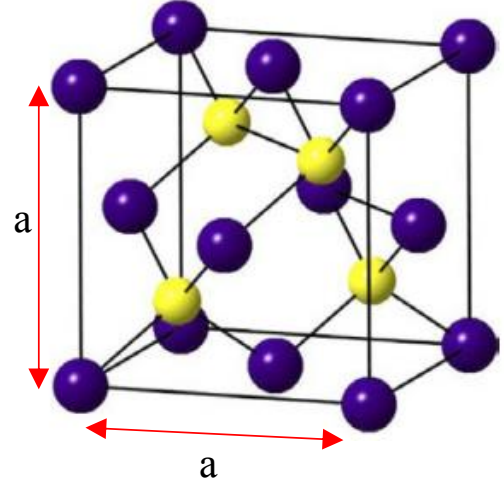

(a)

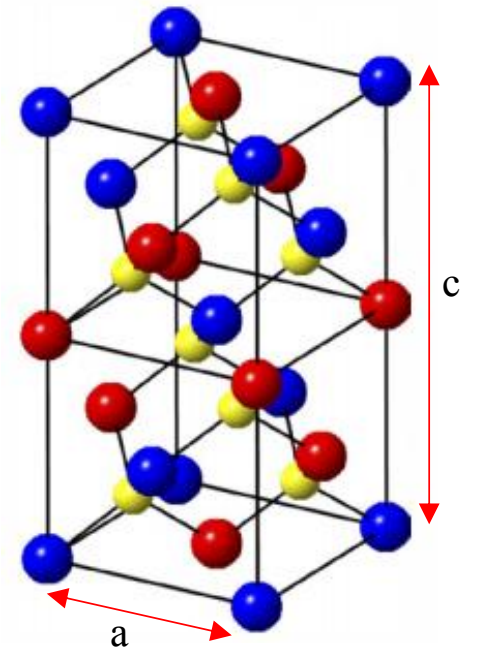

(b)

Figure 2.17. (a) zincblende crystal structure, purple spheres represent metallic atoms and yellow spheres are non-metallic atoms. (b) chalcopyrite crystal structures, red and blue spheres represent the metallic atoms and yellow spheres are non-metal atoms. The figure is taken from [148] 
The diamond structure consists of two interpenetrating face-centered cubic lattices with a basis of two identical atoms at $(0,0,0)$ and $(1 / 4,1 / 4,1 / 4) a$. Silicon and Germanium are found in this structure. The chalcopyrite crystal structure can be considered as a superlattice of zincblende structure in which the group III cation and the group V anions occupy each of the interpenetrating sublattices of the diamond structure. In ternary chalcopyrite crystals, the cations are substituted by two types of cations, one of a higher valence and another of a lower valence.

Figure 2.17 shows the zincblende crystal structure and chalcopyrite crystal structure [149]. In the $\mathrm{ABC}_{2}$ compound, group $\mathrm{A}$ cation and group $\mathrm{B}$ cation stack in $\mathrm{A} \mathrm{B} \mathrm{A} \mathrm{B} \ldots$ sequence with a $c / 2$ separation parallel to the $\mathrm{c}$-axis whereas A A A... and B B B... sequence parallel to the aaxis. Each metallic cation is covalently bonded to four nonmetallic atoms with $\mathrm{sp}^{3}$ bonding. Each anion forms four bonds with two different cations. Therefore, cations form $\mathrm{A}-\mathrm{C}_{4}$ and $\mathrm{B}-\mathrm{C}_{4}$ tetrahedra. Furthermore, each anion and cation have twelve second nearest neighbors. The volume of the unit cell of the chalcopyrite structure is doubled $(c=2 a)$ compared to the zincblende crystal structure if the bonding lengths are equal. The tetragonal aspect ratio $\eta=c / 2 a$ is equal to 1 for the ideal situation. However, the anion atom in chalcopyrite crystal is bonded to two types of cations with different chemical properties and unequal bonding lengths. Hence the structure is distorted along the $\mathrm{c}$ axis result in a shift of the anion from the $(1 / 4,1 / 4,1 / 4) a$ position and $\eta<1$.

This dissertation focuses on CGP, ZGP, and CSP bulk chalcopyrite semiconductors. ZGP crystal is a positive uniaxial crystal with lattice constant $a=5.466 A^{\circ}, c=10.722 A^{\circ}$ and a direct bandgap of $2.34 \mathrm{eV}(530 \mathrm{~nm})$ [150]. It has a wide transparency range from 0.74 to $12 \mu \mathrm{m}$ and a low absorption coefficient in the $\mathrm{THz}$ range. Due to its high nonlinear coefficient $\left(d_{14}=75 \mathrm{pm} / \mathrm{V}\right)$, sufficiently large birefringence, very high melting point, thermal conductivity, and high hardness, ZGP crystal has been recognized as a promising candidate for applications in NLO devices, frequency doublers, and high power optical parametric oscillators (OPOs). Moreover, ZGP crystal is well suited for mid-infrared frequency conversion [70].

CSP is a negative uniaxial crystal with lattice constants $a=5.68 A^{\circ}$ and $c=10.431 A^{\circ}$. Among all the II-IV-V $\mathrm{V}_{2}$ family this crystal has the largest tetragonal distortion and smallest value of $\eta=0.918$. It has a high nonlinear coefficient of CSP $\left(d_{36}=84.5 \mathrm{pm} / \mathrm{V}\right)$ and broad optical transparency range between $0.5-10 \mu \mathrm{m}[32]$. Hence it has been used for numerous frequency 
conversion applications. The bandgap of this material is $2.45 \mathrm{eV}(506 \mathrm{~nm})$ and the refractive index is 3.0 all over its transparency range, at room temperature. CSP has potential applications in infrared mixing, optical parametric processes, nonlinear harmonic generation, and high average power technologies [74]. Moreover, CSP crystals are also used for generating and detecting broadband $\mathrm{THz}$ pulses $[47,71]$.

CGP is a positive uniaxial crystal with lattice constant $a=5.46 A^{\circ}, c=10.76 A^{\circ}$ and direct bandgap of $1.72 \mathrm{eV}$ [150]. It has a nonlinear coefficient of $\left(d_{36}=109 \mathrm{pm} / \mathrm{V}\right)$ and optical transparency range of 0.7-14 $\mu \mathrm{m}$ [135]. Recently there has been much interest in the use of CGP in optical parametric oscillators and upconverters. Also, it has been explored for spintronic applications [135].

Table 2.3. Properties of CGP, ZGP, and CSP chalcopyrite semiconductors.

\begin{tabular}{|l|c|c|c|}
\hline \multicolumn{1}{|c|}{ Material Property } & CGP & ZGP & CSP \\
\hline Birefringence $n_{e}-n_{o}$ & $+0.017[54]$ & $+0.04[61]$ & $-0.05[74]$ \\
\hline Thermal Conductivity $(\mathrm{W} / \mathrm{m} \mathrm{K})$ & $11[33]$ & $35[61]$ & $13.6[74]$ \\
\hline Harness $(\mathrm{kg} / \mathrm{mm} 2)$ & $850[33]$ & $980[33]$ & $930[74]$ \\
\hline Melting Point $\left({ }^{\circ} \mathrm{C}\right)$ & $776[33]$ & $1027[61]$ & $1133[74]$ \\
\hline
\end{tabular}

\subsubsection{Tauc plot to determine absorption edges}

Linear optical transmission measurements on CGP, ZGP, CSP samples are performed with Fourier transformed infrared and UV-VIS spectrometers in ambient conditions. Transmission through samples $\left(I_{\text {sam }}\right)$ and background $\left(I_{o}\right)$ were acquired and absorption $A$ was calculated by $A=-\log \left(I_{\text {sam }} / I_{o}\right)$. To identify the absorption edges for each crystal, the absorption coefficient $\alpha$ is converted into Tauc plots as follows

$$
\alpha h v=A\left(h v-E_{g}\right)^{1 / n}
$$


where $E_{g}$ is the bandgap, $h v$ is the photon energy and $A$ is a proportionality constant. The $n$ depends on the nature of the electronic transition. $n$ is $1 / 2$ for the direct allowed transitions and it is 2 for indirect transitions. Figure 2.19 (b) shows the Tauc plot of linear spectra for all three samples. It can be observed that the absorption is very low for all three crystals at the low photon energy. A strong absorption edge can be observed at $1.70 \mathrm{eV}, 1.97 \mathrm{eV}$ and $2.12 \mathrm{eV}$ for CGP, ZGP and CSP. These three values are consistently lower than previous measurements of the band gaps $\left(E_{g}\right)$. This may be due to the samples are thicker than the optical penetration depth at $E_{g}$

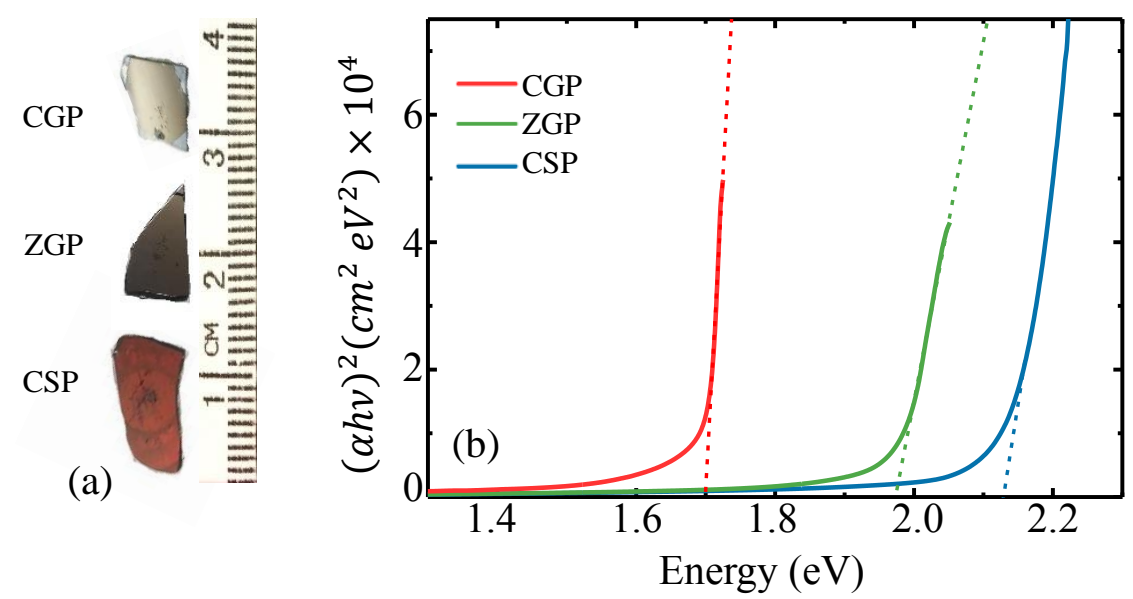

Figure 2.18 (a) Photographs of the polished CGP, ZGP, and CSP crystals from top to bottom. Visible light is reflected from the CGP. (b) Tauc plot for absorption edges using direct-gap normalization. Figure modified from Piyathilaka et al. [47] 


\section{Chapter 3: Ground-State AC Conductivity}

\subsection{Introduction}

A detailed understanding of the microscopic origins of phenomenological parameters such as refractive index and $\mathrm{AC}$ conductivity is vital for characterizing optical materials and their devices. This allows for feedback to improve growth and/or processing. In particular, AC conductivity measured at far-infrared or $\mathrm{THz}$ frequencies can provide information about the mechanisms of charge transport in the material. Moreover, since the optical and electrical properties of semiconductors are greatly influenced by the temperature of the lattice, knowledge of the temperature dependence of these properties is essential to understand underlying scattering mechanisms.

The traditional contact-based Hall-effect measurement is a powerful tool, commonly used to investigate electrical transport. It provides a wealth of information about materials and their devices, classifying microscopic transport mechanisms, and even distinguishing classical or quantum transport behavior. Over the past few decades, the enormous improvement in contactless Terahertz Time-Domain Spectroscopy (THz-TDS) [151-153] has opened a window of accurate noninvasive optical and transport data for liquids, gases and solids [99-101,154,155].In the latter, THz-TDS is used to provide feedback for material growth and measure device performance. $\mathrm{THz}$ pulses measure carrier dynamics on the picosecond time scale [139] through low-energy intraband excitation of free carriers with comparable magnitudes of conductivity to conventional Hall-effect measurements [142].

There is still much to understand about models of conduction and their effect at $\mathrm{THz}$ frequencies, requiring the application of THz-TDS to simply samples such as bulk crystals. One material class that has been interesting to the optics community is II-IV-V 2 chalcopyrite crystals. While optical properties are well known in these materials, there have been no prior systematic studies of temperature-dependent $\mathrm{THz}$ dispersion and $\mathrm{AC}$ conductivity. Hence, chalcopyrite crystals are model systems for exploring temperature-dependent THz-TDS acquisition of microscopic electronic transport phenomena by applying models that have traditionally been applied to Hall-effect measurements as a function of temperature. 
In this chapter, temperature-dependent THz-TDS is reported for CGP, ZGP, and CSP in the $\mathrm{THz}$ region over $4 \mathrm{~K}-300 \mathrm{~K}$ temperature range. It is found that the refractive index increases with the increasing temperature associated with greater electron-phonon coupling. The temperature dependence of the refractive index is discussed using the Bose-Einstein model. By Fitting complex AC conductivity to the Drude-Smith model, the carrier density and relaxation time are obtained. The temperature dependence of the carrier density is described by the Fermi model for carrier occupancy. The carrier mobility is determined from the reciprocal addition of extracted carrier scattering times, giving temperature-dependent mobility that is described by a combination of polar optical phonon, acoustic phonons, deformation potential, ionized impurity, and dislocation scattering.

\subsection{Extracting Complex Material Parameters}

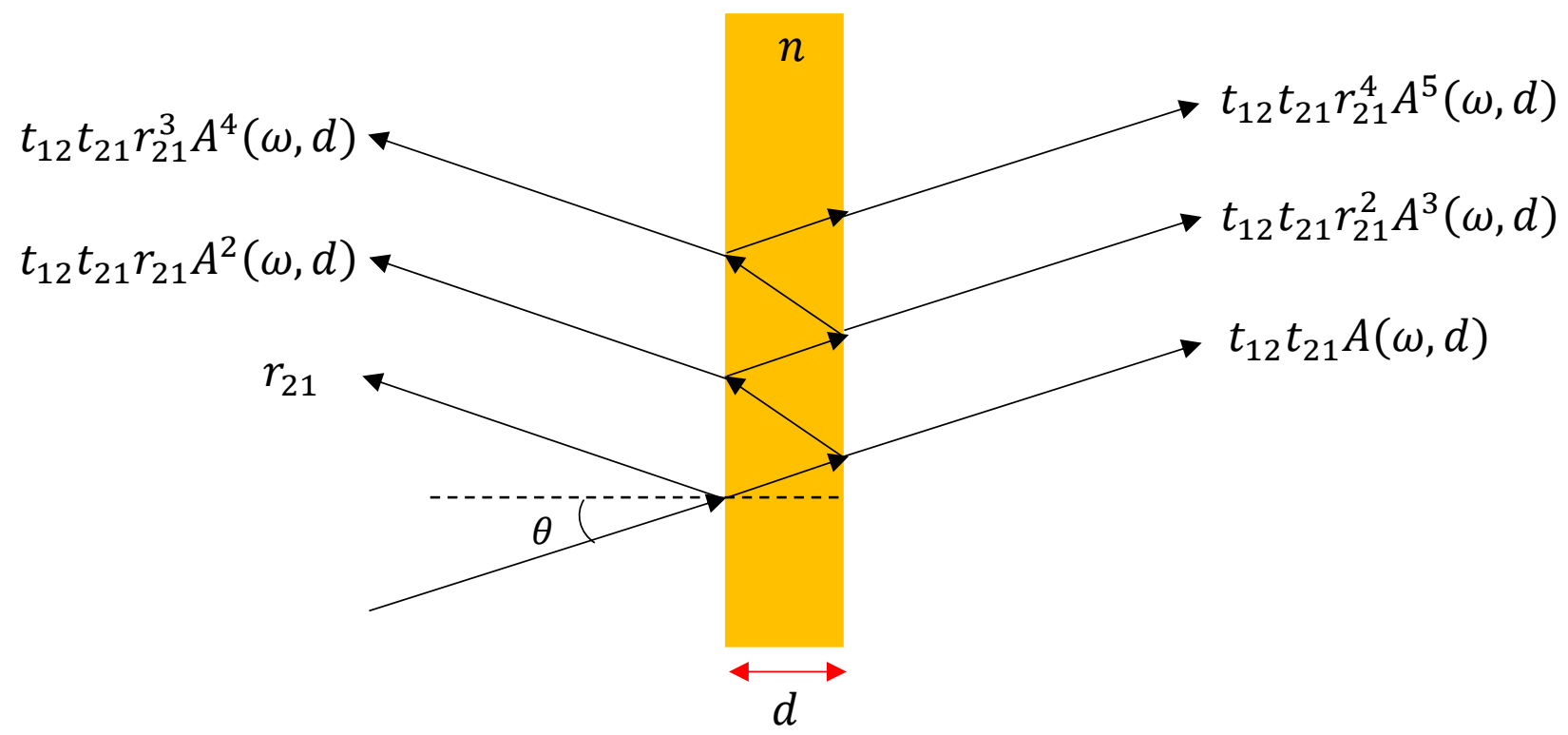

Figure 3.1. Transmission of an electromagnetic wave through a material with a thickness $d$ and refractive index $n(\omega)$

As discussed in section 2.7, THz-TDS is a powerful technique to determine the complex optical constants of materials. In this technique, both the amplitude and phase of the THz electric field are recorded in the time domain. In a typical experiment, two measurements are performed. Firstly, a reference measurement without a sample is conducted. Then the second measurement is 
performed with a sample in the THz path. This allows one to obtain complex optical parameters by comparing the spectral profile of the two measurements. This section will outline the basic procedure and theories for obtaining complex optical parameters from the THz-TDS measurements.

When $\mathrm{THz}$ radiation incident on a material, some of the radiation is transmitted and part of the radiation is reflected. Inside the sample, multiple reflections can occur at the interfaces. Figure 3.1 illustrates the schematic of the interaction of the $\mathrm{THz}$ pulse with the sample with a thickness $d$. The total transmission is the superposition of all the parts having endured multiple reflections. The total transmitted field can be expressed as follows $[152,156]$

$$
\begin{gathered}
E_{T}=E_{I} t_{12} t_{21} A(\omega, d)+E_{I} t_{12} t_{21} A(\omega, d)^{3} r_{21} r_{12}+E_{I} t_{12} t_{21} A((\omega, d))^{5} r_{12}^{2} r_{21}^{2}+\cdots \\
=E_{I} t_{12} t_{21} A(\omega, d) \sum_{p=0}^{P}\left\lfloor r_{12} r_{21} A(\omega, d)\right\rfloor^{P}
\end{gathered}
$$

where $A(\omega, d)=\exp [i \tilde{n}(\omega) \omega d / c]$ is the propagation factor, $t_{12}$ and $t_{21}$ are the Fresnel transmission coefficients: $t_{12}(\omega)=2 / \tilde{n}(\omega)+1, t_{21}(\omega)=2 \tilde{n}(\omega) / \tilde{n}(\omega)+1, r_{12}$ and $r_{12}$ are the Fresnel reflection coefficients: $r_{21}=r_{12}=\tilde{n}(\omega)-1 / \tilde{n}(\omega)+1$ and $E_{I}$ is the incident pulse. By substituting the propagation factor in equation 3.1 the transmission becomes

$$
E_{T}=E_{I} t_{12} t_{21} \exp \left[i \frac{\omega d}{c} \tilde{n}(\omega)\right] F(\omega),
$$

where $F(\omega)$ is the Fabry-Perot term which describes reflections within the sample.

$$
F(\omega)=\sum_{p=0}^{p}\left[r_{12} r_{21} \exp (i 2 \tilde{n}(\omega) \omega d / c)\right]^{P}
$$

The complex transmission coefficient $T(\omega)$ can be obtained by substituting Fresnel coefficients as follow

$$
T(\omega)=\frac{4 \tilde{n}(\omega) \exp (i \omega d \tilde{n}(\omega) / c)}{[\tilde{n}(\omega)+1]^{2}} F(\omega) .
$$


When performing THz-TDS, two sample limits are considered: optically thick samples and optically thin samples. If the sample thickness is larger than the THz wavelength, $\left(d \gg \lambda_{T H z}\right)$, Fabry- Perot effect cannot be observed. Then $P \rightarrow 0$ and equation 3.4 can be further simplified as follows

$$
T(\omega)=\frac{4 \tilde{n} \exp (i \omega d(\tilde{n}-1) / c)}{(1+\tilde{n})^{2}}
$$

Let us consider the procedure for obtaining complex transmission coefficient $T(\omega)$ from THz-TDS measurements. Both the phase and magnitude of the $\mathrm{THz}$ electric field through the sample, $E_{S}(\mathrm{t})$, and free space, $E_{0}(t)$, are measured to obtained $T(\omega) . T(\omega)$ is obtained from the Fourier transform of the $\mathrm{THz}$ electric field through the sample, $E_{s}(\omega)$, and free space, $E_{0}(\omega)$, giving

$$
T(\omega)=\frac{E_{s}(\omega)}{E_{0}(\omega)}=A \exp (i \phi)
$$

where $A(\omega)$ and $\phi(\omega)$ are the amplitude and the phase of the complex transmission coefficient. Using $\tilde{n}=n+i \kappa$ and equating equation 3.5 and 3.6 following relationship can be obtained.

$$
A \exp (i \phi)=\frac{4(n+i \kappa) \exp [i \omega d(n-1) / c] \exp (-\omega d \kappa / c)}{[(1+n)+i \kappa]^{2}}
$$

Now the complex components can be replaced by their magnitude and phase

$$
A \exp (i \phi)=\frac{4 \sqrt{n^{2}+\kappa^{2}} \exp [i \arctan (\kappa / n)] \exp [i \omega d / c(n-1)] \exp (-\omega d \kappa / c)}{\left\{\sqrt{(1+n)^{2}} \exp [i \arctan (\kappa /(n+1))]\right\}^{2}}
$$

It can be implemented the assumption that $\kappa<<n$

$$
\operatorname{Aexp}(i \phi)=\frac{4(n+i \kappa) \exp \left[\frac{i \omega d}{c}(n-1)\right] \exp \left(-\frac{\omega d}{c} \kappa\right)}{((1+n)+i \kappa)^{2}}
$$

Now the magnitudes and phases can be matched, 


$$
\begin{gathered}
\phi=\frac{\omega d}{c}(n-1) . \\
A=\frac{4 n \exp \left(-\frac{\omega d}{c} \kappa\right)}{(1+n)^{2}} .
\end{gathered}
$$

The real part of the refractive index $n(\omega)$ and extinction coefficient $\kappa(\omega)$ can be extracted from equation (3.10) and equation (3.11). The real part of the refractive index is related to the phase difference between incident and transmitted field whereas the extinction coefficient is related to the absorption of the sample [157].

$$
\begin{gathered}
n(\omega)=1+\frac{c}{\omega d} \phi(\omega) \\
\kappa=\frac{-c}{\omega d} \ln \left[\frac{A(1+n)^{2}}{4 n}\right]
\end{gathered}
$$

One can obtain the complex dielectric function $\tilde{\epsilon}(\omega)$ of the medium using the complex refractive index as follows

$$
\begin{gathered}
\tilde{n}(\omega)^{2}=\tilde{\epsilon}(\omega)=\epsilon^{\prime}(\omega)+\epsilon^{\prime \prime}(\omega) \\
\epsilon^{\prime}=n^{2}-\kappa^{2} \\
\epsilon^{\prime \prime}=2 n \kappa
\end{gathered}
$$

where $\epsilon^{\prime}(\omega)$ is the real part and $\epsilon^{\prime \prime}(\omega)$ is the imaginary part of the dielectric function. The complex AC conductivity $\tilde{\sigma}(\omega)$ is related to the dielectric function as follows

$$
\tilde{\epsilon}(\omega)=\tilde{\epsilon}(\infty)+i \frac{\tilde{\sigma}(\omega)}{\omega \varepsilon_{0}}
$$

where $\tilde{\epsilon}(\infty)$ is the dielectric constant in the high-frequency limit. In the low-frequency limit, the dielectric function is related to the motion of bound charges and $\mathrm{AC}$ conductivity is related to the free charges. However, the response of the bound and free charges at optical frequencies is significantly different from the low-frequency case. Rearranging equation (3.17) and expanding the complex conductivity to be $\tilde{\sigma}(\omega)=\sigma_{1}(\omega)+i \sigma_{2}(\omega)$ allows for the determination of the real and imaginary parts. 


\subsection{THz Transmission}
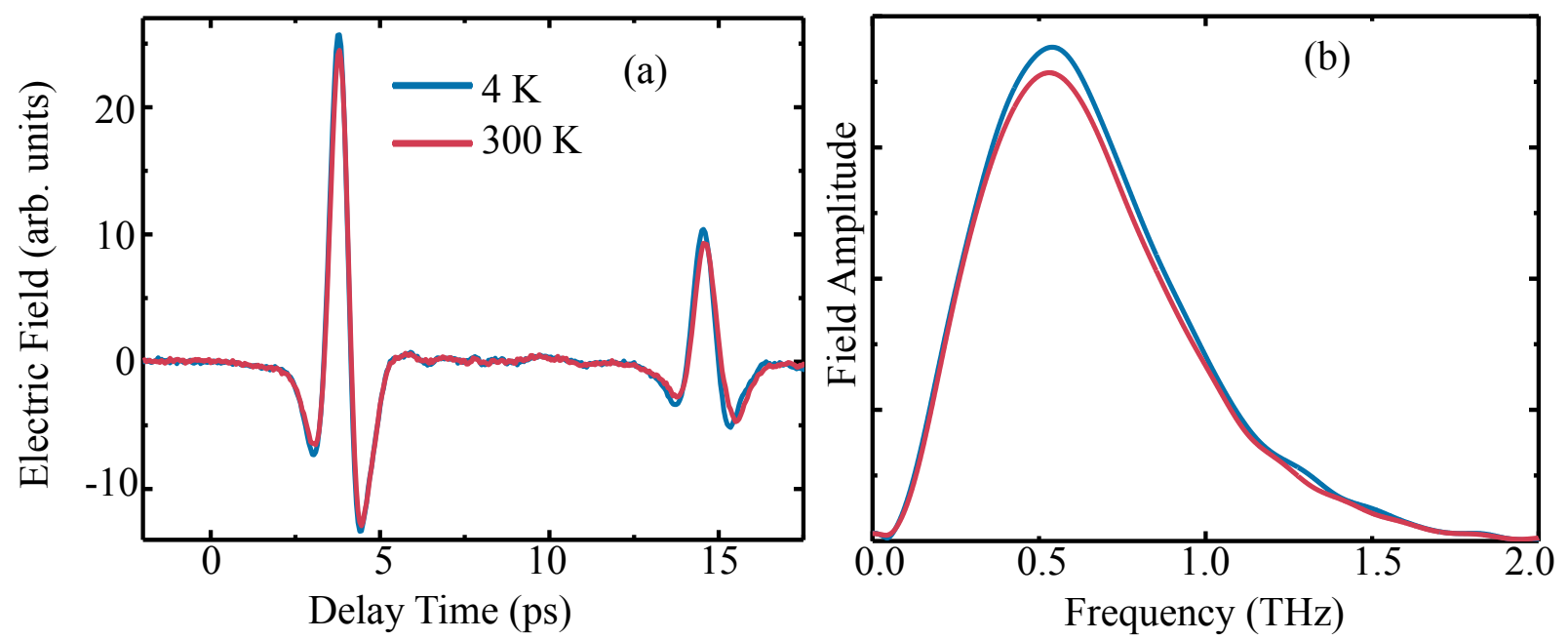

Figure 3.2 (a). Transmitted $\mathrm{THz}$ electric field through CSP sample at $4 \mathrm{~K}$ and $300 \mathrm{~K}$. (b). Amplitude spectra in the frequency domain after applying the Fourier transform.

Temperature-dependent THz-TDS is performed as described in section 2.7. THz electric field transmitted through cryostat with no sample is measured by varying the temperature $4 \mathrm{~K}-300$ K. Then THz transmitted electric field through CGP, ZGP, and CSP samples are measured over the temperature range of $4 \mathrm{~K}$ to $300 \mathrm{~K}$. Figure 3.2 (a) shows typical $\mathrm{THz}$ electric field transmitted through CSP sample at $4 \mathrm{~K}$ and $300 \mathrm{~K}$. Time-domain results are treated with an arctan window function to remove the first reflection in ZnTe EO crystal and zero-padded. Then FFT is performed on the experimental transients. The FFT allows one to obtain spectral information such as amplitude and phase of the sample. Figure 3.2 (b) shows the amplitude of the FFT of the transmitted electric field through CSP sample at $4 \mathrm{~K}$ and $300 \mathrm{~K}$. The useful frequency range of the transmitted terahertz electric field exists in $0.2 \mathrm{THz}-1.5 \mathrm{THz}$. The observed decrease in $\mathrm{THz}$ transmission with increasing temperature is due to the increase in free-carrier concentration at higher temperatures. This will result in higher absorption at elevated temperatures.

Figure 3.3 (a) shows typical time-domain results from CGP, ZGP, and CSP at $300 \mathrm{~K}$ compared to a reference signal with no sample in the cryostat. The transmitted $\mathrm{THz}$ pulse experiences absorption and delay due to its interaction with the sample. Figure 3.3 (b) shows the corresponding respective field amplitude spectra from the numerical Fourier transform, which can 
be obtained without taking into consideration of the large transmission delay of the pulse through the samples.
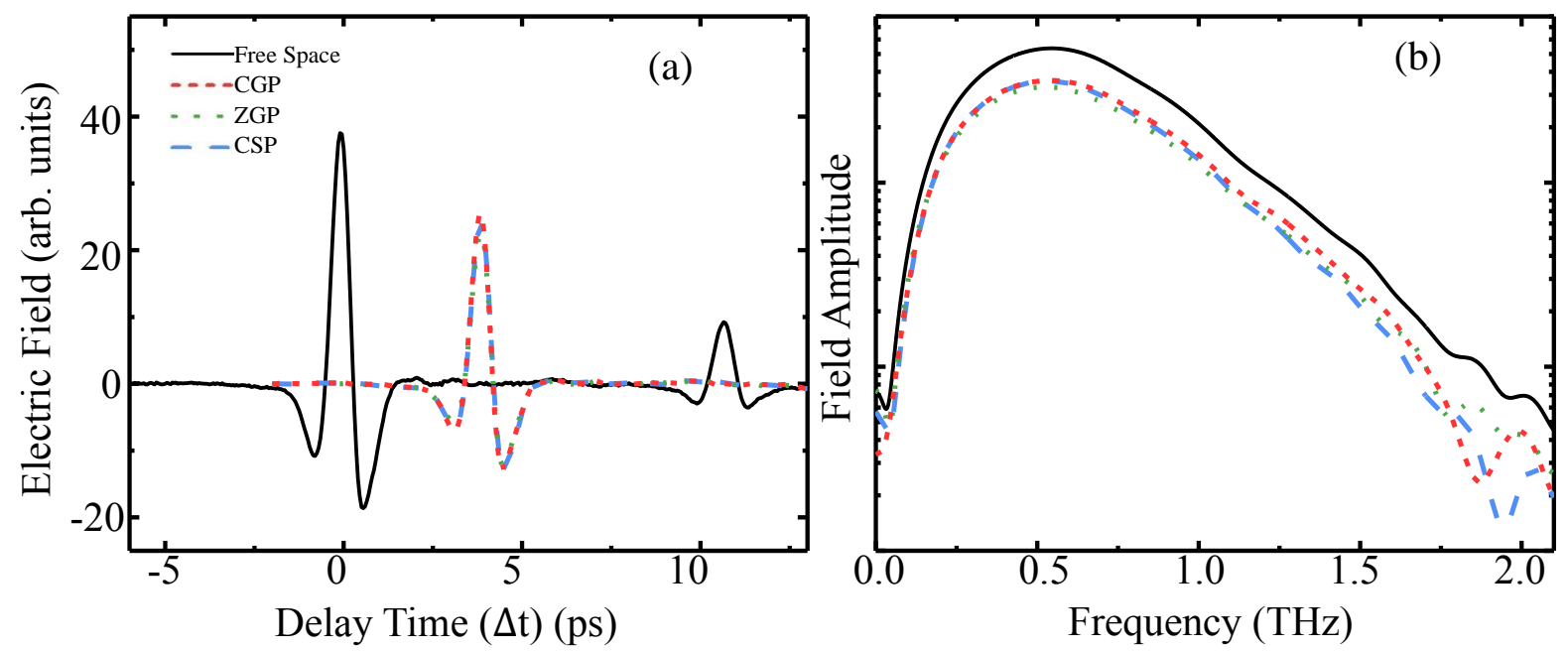

Figure 3.3. (a) The typical time-domain transient of terahertz propagating through free space (between source and detector) or transmitted through CGP, ZGP, and CSP at 300 K.(b) Corresponding amplitude spectra in the frequency domain. Figure modified from Sooriyagoda $e t$ al. [158]

\subsection{THz Refractive Index}

The optical properties of a material are determined by the microscopic and macroscopic nature of that material. Most of the optical properties including transmission, absorption, refraction, and reflection are associated with the refractive index and extinction coefficient. Moreover, electronic properties such as polarizability and dielectric constant depend on the refractive index of the materials. The complex refractive index of solids is determined by electronic transitions, lattice vibrations, and free carrier concentration. However, the dominant process is decided by the type of material and spectral region of interest. Electronic transitions contribute to the complex refractive index of all types of materials. Characterization of lattice vibrations is also required to fully investigate the optical properties of semiconductors and insulators.

Additionally, free carriers influence the transparency and absorption of semiconductors. Consequently, the optical properties of metals and semiconductors in the visible and infrared regions are determined by the free-carrier concentration. 
The knowledge of the material refractive index is crucial in optical or optoelectronic applications. In particular, devices such as waveguides, solar cells, detectors, optoelectronic switches, photonic crystals, laser diodes, and laser techniques require pre-knowledge of the refractive index. Also, application-specific coating technologies depend on the spectral properties of substances [159].
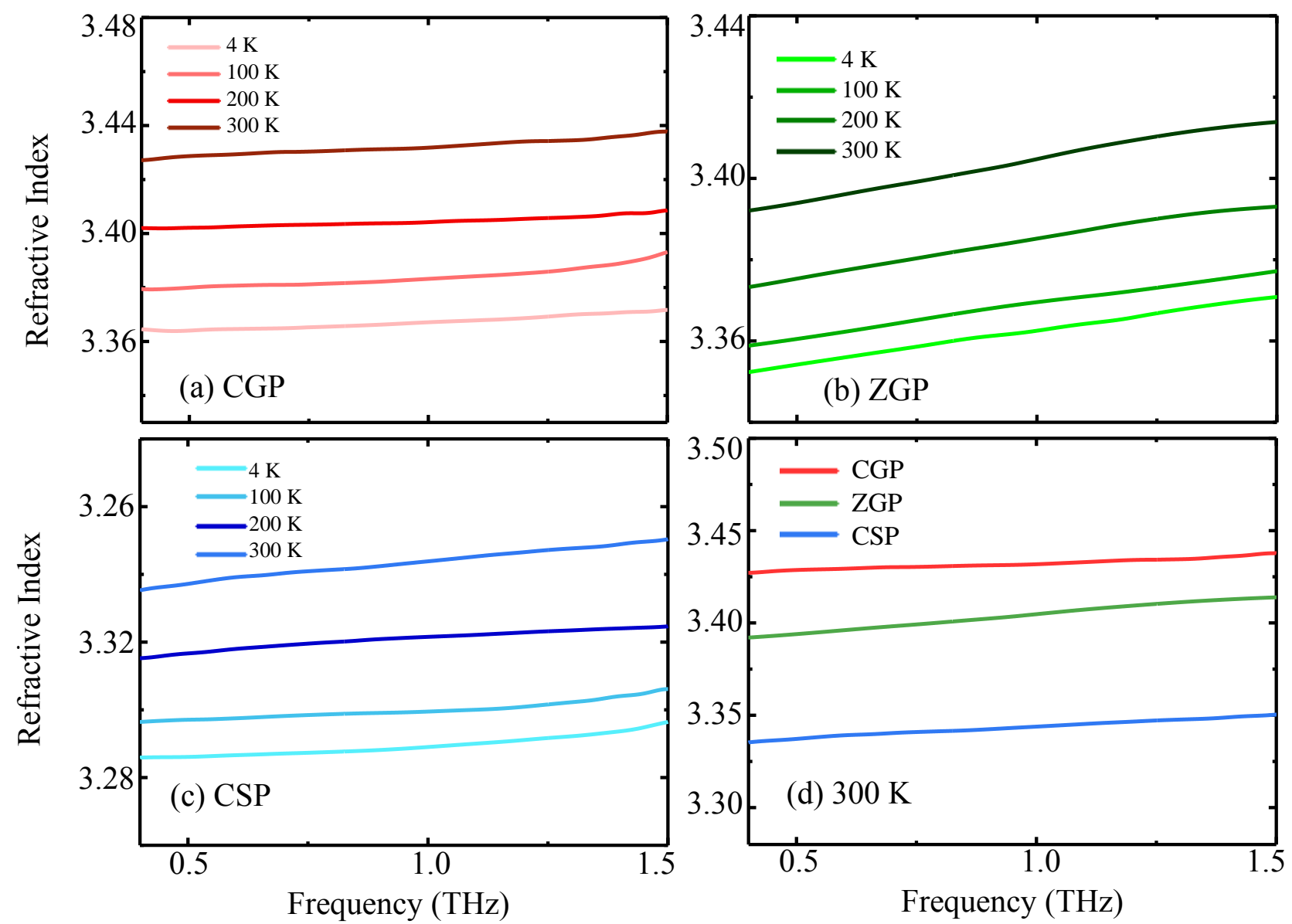

Figure 3.4. THz dispersion of refractive index for (a) CGP (b) ZGP (c) CSP for different temperatures. (d) refractive index for all three crystals at $300 \mathrm{~K}$

The complex refractive index can be determined from the $\mathrm{THz}$ transmission coefficient. The detailed procedures of the data extraction and analysis are given in section 3.2. Figure 3.4 shows the real part of the refractive index of (a) CGP (b) ZGP and (c) CSP crystals in the frequency range $0.4-1.5 \mathrm{THz}$ for a series of temperatures. Figure 3.4 (d) shows the temperature-dependent refractive index for all three crystals at $300 \mathrm{~K}$. Among all three crystals CGP sample shows the 
highest refractive index. It can be seen that the refractive index increases monotonically with frequency, although more significantly with temperature.

\subsubsection{The Temperature Dependence of the Refractive Index}
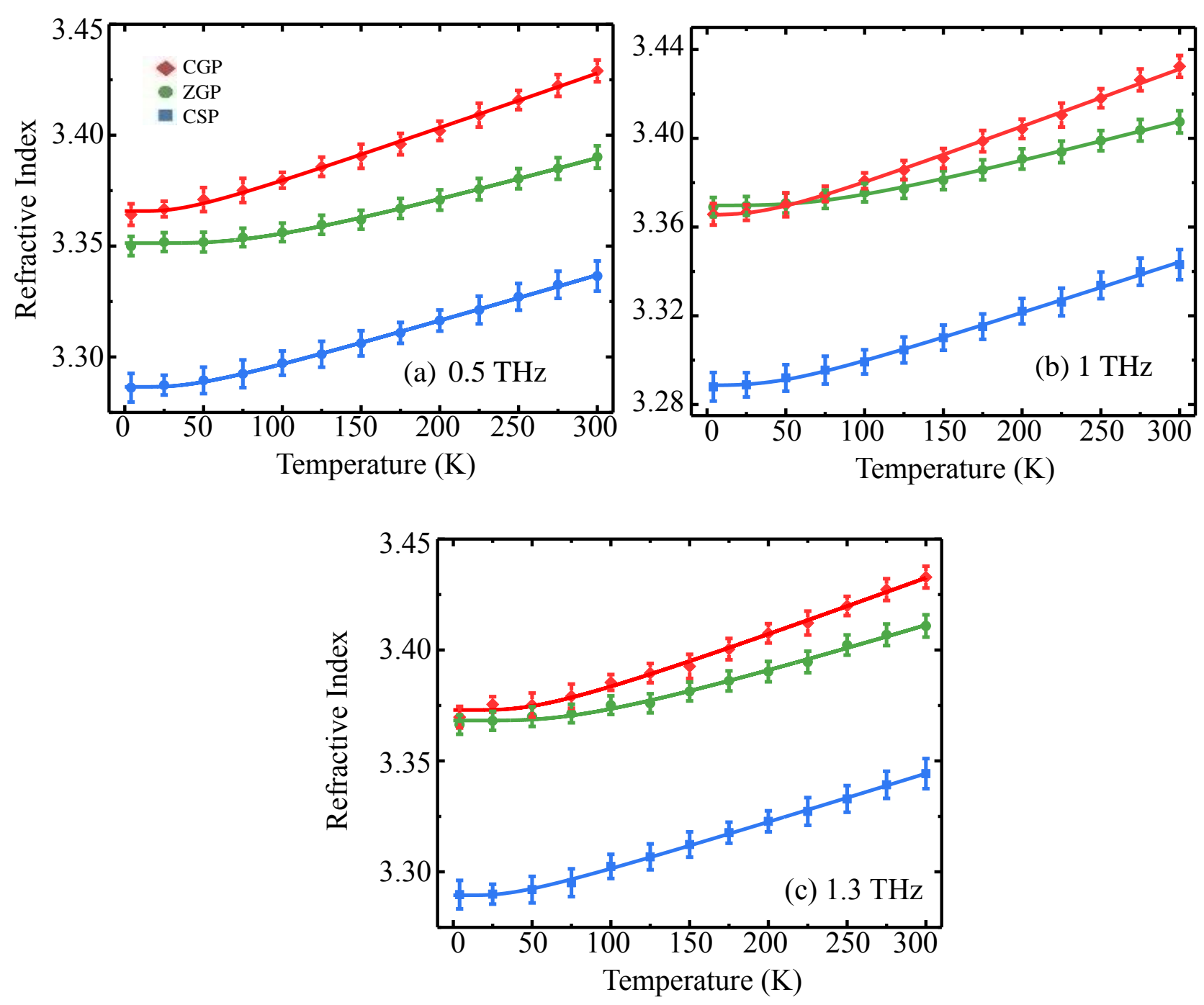

Figure 3.5. The temperature-dependent refractive index at (a) $0.5 \mathrm{THz}$ (b) $1 \mathrm{THz}$ (c) $1.3 \mathrm{THz}$ for all three crystals, fit with a Bose-Einstein model.

As shown in figure 3.4, the refractive index is greatly influenced by temperature. The changes of the refractive index with temperature are due to the modification of the refractive index by electrons and phonons at different temperatures [160]. Electron-phonon interactions increase at 
higher temperatures and this will result in a higher refractive index. This temperature dependence can be described by the Bose-Einstein model [161,162]

$$
n(T)=n_{0}+S_{n}\left[\frac{1}{\exp \left(\hbar \omega_{p n} / k T\right)-1}+\frac{1}{2}\right],
$$

where $n_{0}$ is the refractive index approaching absolute zero $(0 \mathrm{~K}), S_{n}$ is the dimensionless electronphonon coupling constant, $k$ is the Boltzmann constant and $\omega_{p n}$ is the effective phonon frequency. $\exp \left(\hbar \omega_{p n} / k T\right)-1$ is the Bose-Einstein factor for an effective phonon frequency $\omega_{p n}$ which models the vibrational degrees of freedom to modify $n(T \rightarrow 0)$ where $\hbar \omega_{p n}>k T$. Figure 3.5 shows the refractive index as a function of temperature for all crystals at frequencies (a) $0.5 \mathrm{THz}$ (b) $1 \mathrm{THz}$ and (c) $1.3 \mathrm{THz}$. It can be seen that the experimental refractive index values are fit well by the Bose-Einstein model, as seen by the data points that overlay the solid lines. The parameters $S_{n}, \omega_{p n}$ and $n_{0}$ are obtained using the Bose-Einstein fitting for all three crystals.

Table 1. Molar masses of atoms

\begin{tabular}{cc}
\hline Atom & Molar mass (g/mol) \\
\hline $\mathrm{Cd}$ & 112.414 \\
$\mathrm{Ge}$ & 72.630 \\
$\mathrm{Zn}$ & 65.38 \\
$\mathrm{Si}$ & 28.085 \\
$\mathrm{P}$ & 30.974 \\
\hline
\end{tabular}

Figure 3.6 shows the resulting $S_{n}\left(\omega_{p n} / 2 \pi\right)$ revealing a linear relationship between the electron-phonon coupling with the effective phonon energy at (a) $0.5 \mathrm{THz}$ (b) $1.0 \mathrm{THz}$ and (c) 1.3 $\mathrm{THz}$ frequencies. Crystals fall on the line based on the lightest A-site cation having the highest frequency and strongest coupling, and the B-site cation influencing the frequency and coupling for the crystals with the heavier A-site cation crystal. Table 1 shows the atomic molar masses. For CSP crystal electron-phonon coupling constant is about $0.027-0.032$, for CGP crystal it is about 
0.027-0.042 and for ZGP crystal 0.043-0.052. ZGP crystal shows higher electron-phonon coupling among all three crystals at all frequencies and for comparison $S_{n}=0.019$ in diamond [162]. ZGP has the highest average phonon frequencies compare to the other two crystals. Table 2 shows the extracted $n_{0}$ at $0.5 \mathrm{THz}, 1 \mathrm{THz}$ and $1.3 \mathrm{THz}$.
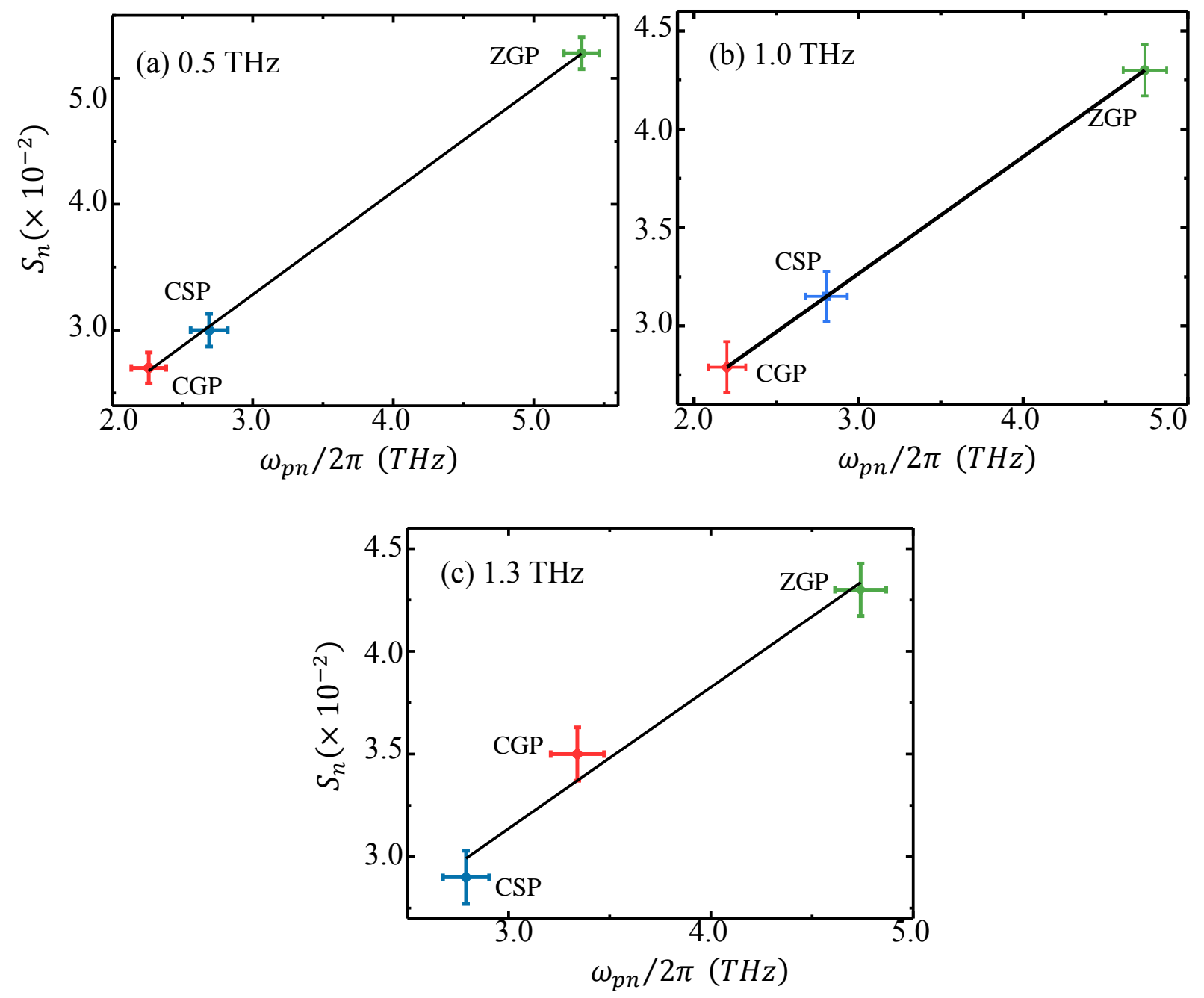

Figure 3.6. Dependence of the extracted coupling constant versus the extracted average phonon energy with a linear fit at (a) $0.5 \mathrm{THz}$ (b) $1 \mathrm{THz}$ (c) $1.3 \mathrm{THz}$ 
Table 2. Extracted $n_{0}$ values from the Bose-Einstein model for three crystals

\begin{tabular}{cccc}
\hline & $n_{0}(v=0.5 \mathrm{THz})$ & $n_{0}(v=1 \mathrm{THz})$ & $n_{0}(v=1.3 \mathrm{THz})$ \\
\hline CSP & 3.27 & 3.27 & 3.28 \\
ZGP & 3.33 & 3.35 & 3.35 \\
CGP & 3.35 & 3.35 & 3.35 \\
\hline
\end{tabular}

\subsubsection{The Band Gap Dependence of the Refractive Index}

Understanding the relationship between refractive index and band gap of semiconductors is vital for their device applications. The refractive index of a semiconductor generally decreases with increasing band gap energy. Different types of theoretical models have been proposed to describe the relationship between band gap and refractive index. In 1950, Moss [163] proposed that the electron energy levels in a solid are scaled by a factor of $1 / \epsilon_{\text {eff }}^{2}$ or $1 / n^{4}$ where, $\epsilon_{\text {eff }}$ is the dielectric constant and $n$ is the refractive index. According to this theory, the refractive index for a specific class of semiconductors can be expressed as $n=\left(K / E_{g}\right)^{1 / 4}$ where $E_{g}$ is the band gap energy and $K$ is a constant equal to $95 \mathrm{eV}$. Ravindra et al. [164] have modified the Moss theory and proposed an empirical relation for the refractive index for semiconductors, $n=4.084+\beta E_{g}$, where $\beta=-0.62 \mathrm{eV}^{-1}$. The major drawback of this model is it indicates that the materials with $n \geq 4.084$ cannot occur. Herve and Vandamme [165] used oscillatory theory and proposed a relationship for the refractive index and band gap energy as, $n=\left(1+\left(A / E_{g}+B\right)^{2}\right)^{1 / 2}$ where $\mathrm{A}$ is the hydrogen ionization energy $\approx 13.6 \mathrm{eV}$ and $\mathrm{B}$ is $3.47 \mathrm{eV}$. This model fails to predict the refractive index of materials with low energy gaps $\left(E_{g}<1.4 \mathrm{eV}\right)$. Recently Kumar et al. developed a simple model to calculate the refractive index of I-III- $\mathrm{V}_{2}$ and II-IV $-\mathrm{V}_{2}$ ternary chalcopyrite crystals described as follows [166]

$$
n=K_{1} e^{-K_{2} E_{g}}
$$

where $K_{1}$ and $K_{2}$ are constant. 
Figure 3.7 shows the refractive index of all three crystals at $300 \mathrm{~K}$ as a function of band gap energy at (a) $0.5 \mathrm{THz}$ (b) $1 \mathrm{THz}$ (c) $1.5 \mathrm{THz}$ frequencies. It can be observed that the refractive index decreases with band gap energy. The measured band gap-dependent refractive index is fitted with equation 3.19 shown in figure 3.7 as thick solid lines. It can be seen that the experimental refractive index values are fit well by equation 3.19. Table 03 shows the extracted $K_{1}$ and $K_{2}$ values at frequencies $0.5 \mathrm{THz}, 1.0 \mathrm{THz}$, and $1.5 \mathrm{THz}$. These $K_{1}$ and $K_{2}$ values can be used to determine the bandgap dependence of the refractive index of chalcopyrite crystals.

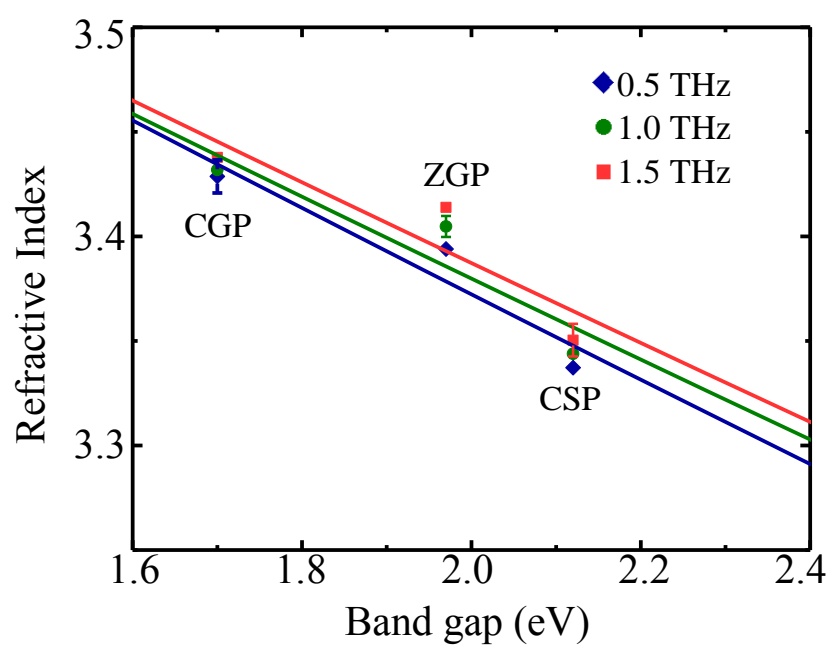

Figure 3.7. Dependence of the refractive index versus the band gap energy with a fit with equation (3.19) at frequencies $0.5 \mathrm{THz}, 1 \mathrm{THz}$ and $1.5 \mathrm{THz}$.

Table 3. Extracted $K_{1}$ and $K_{2}$ values from refractive index vs band gap fittings

\begin{tabular}{ccc}
\hline Frequency $(\mathrm{THz})$ & $K_{1}$ & $K_{2}$ \\
\hline 0.5 & 3.81 & 0.061 \\
1.0 & 3.79 & 0.058 \\
1.5 & 3.79 & 0.057 \\
\hline
\end{tabular}




\subsection{THz Absorption}

The frequency-dependent extinction coefficient can be determined from the $\mathrm{THz}$ transmission coefficient as described in section 3.2. Figure 3.8 (a) shows the THz absorption coefficient, $\alpha(\omega)=2 \omega \kappa(\omega) / c$, determined from the extinction coefficients for (a) CGP, (b) ZGP and (c) CSP at $300 \mathrm{~K}$ In all cases, $\alpha(\omega)$ increases with $\omega$ indicating some peak at frequencies out of the detection range. At $300 \mathrm{~K}$, CSP has a more strongly varying absorption than the other crystals, which competes with a strong background absorption that can be seen to be reduced by cooling. The temperature-dependent absorption results from higher free-carrier concentrations at higher temperatures [167]. Despite the change in backgrounds, the absorption spectra are an indication of a finite frequency mode, most likely due to a phonon mode beyond the upper range of the experiment. As a consequence, a Lorentzian function is used to fit the data, as shown by the dotted lines overlaid on the solid lines. Resonance of forced, damped harmonic oscillators has a Lorentzian line shape. Hence, we use the Lorentzian function to fit our data. Whereas Gaussian or Vogit profiles are used to describe the line shape of inhomogeneous broadening.
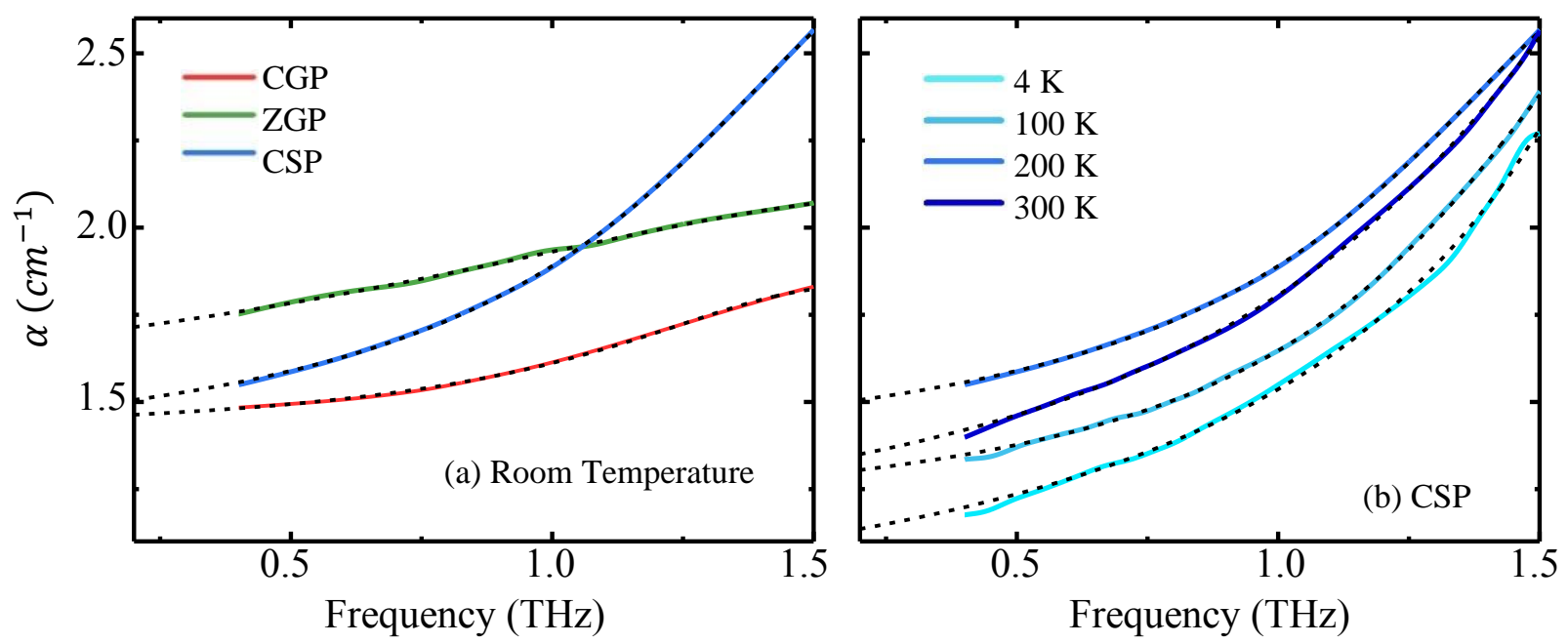

Figure 3.8. Absorption coefficient of (a) CGP, ZGP and CSP at $300 \mathrm{~K}$ and (b) CSP at various temperatures. Figure modified from Sooriyagoda et al. [158] 


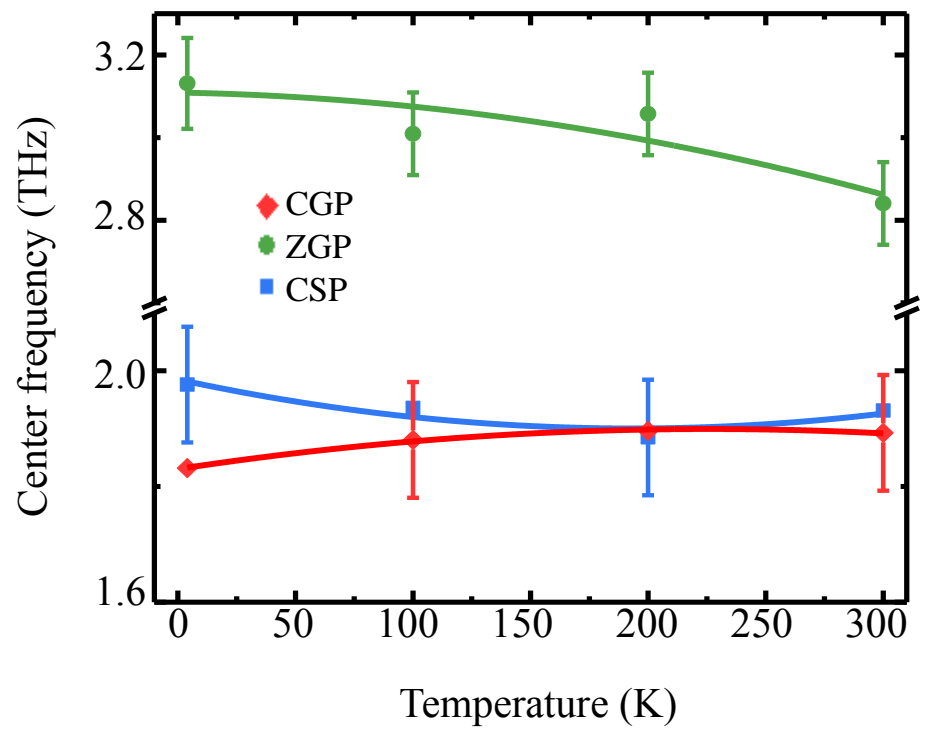

Figure 3.9 Extracted center frequency from the Lorentzian fitting as a function of temperature for all three crystals. Figure modified from Sooriyagoda et al. [158]

Figure 3.9 shows the extracted finite center frequency of the resonance as a function of temperature for all three crystals. It is seen that the phonon frequencies are mildly dependent on temperature. The observed decreasing center frequencies with temperature might be due to a Fano resonance $[167,168]$. The extracted phonon frequencies at room temperature match with those reported from Raman measurements; namely, 1.89 THz for CGP [68], $2.82 \mathrm{THz}$ for ZGP [62,68], and $1.98 \mathrm{THz}$ for CSP [169].

\subsection{Conductivity Models}

When $\mathrm{THz}$ electric field incident on a material it stimulates the motion of free carriers in the system. Hence using THz-TDS electrical conductivity of the sample can be determined as a function of frequency $(\omega)$. Its non-destructive and contactless nature are major advantages of this technique compare to conventional contact-based Hall effect measurements. The conductivity spectrum $\hat{\sigma}(\omega)$ provides the magnitude and spectral shape of the conductivity, giving information about carrier transport mechanisms in the material. Important physical parameters like carrier density, scattering rate, DC conductivity and mobility can be extracted by fitting conductivity 
spectra with an appropriate conduction model. This section will outline and compare various conduction models of the THz conductivity.

\subsubsection{Drude Model}

The Drude model, also known as the Drude-Lorentz model is commonly used to describe the frequency dependence of the complex optical conductivity in metals and semiconductors. This model was first proposed by Paul Drude in 1900. In this model, electron-hole plasma is considered as a non-interacting gas. Charge carriers undergo scattering events that randomize their momentum with a scattering rate. A Semiclassical model of charge transport can be used to derive the Drude response. The motion of electrons under an external electric field is described through a forced damped harmonic oscillator without restoring force as follows

$$
\frac{d^{2} r}{d t^{2}}+\gamma \frac{d r}{d t}=-\frac{e}{m} E(t)
$$

where $r$ is the average displacement of the charge carriers, $e$ is the elementary charge, $m$ is the mass of the charge carrier and $\gamma$ is the scattering rate. Equation 3.20 can be solved to obtain the dielectric response to an external AC electric field

$$
\epsilon_{D}(\omega)=\epsilon_{\infty}-\frac{\omega_{p}^{2}}{\omega^{2}+i \gamma \omega}
$$

where $\epsilon_{\infty}$ is the background dielectric constant, $\omega_{p}=\sqrt{N e^{2} / \varepsilon_{0} m}$ is the plasma frequency with $N$ as the carrier density. If $\omega \ll \omega_{p}$, the well-known Drude model can be obtained by expressing the dielectric response in terms of the complex conductivity

$$
\sigma_{1}(\omega)+i \sigma_{2}(\omega)=\sigma(\omega)=\frac{N e^{2} \tau / m}{1-i \omega \tau}
$$

where $\tau$ is the scattering time - the average time between consecutive scattering events. 


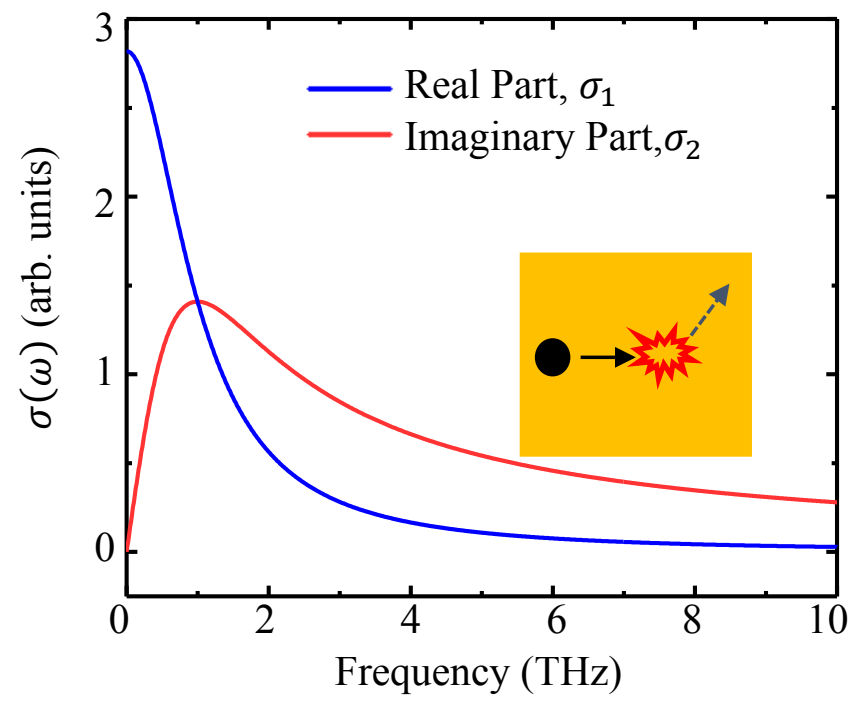

Figure 3.10. The real and imaginary part of the conductivity spectra determined using the Drude model with $N=1 \times 10^{16} \mathrm{~cm}^{-3}$ and $\tau=1 p s$.

Figure 3.10 shows the conductivity spectrum of the Drude model. The real part of the conductivity reaches its highest value at direct current (DC) and decreases at higher frequencies. The imaginary part of the conductivity is always positive and has a maximum at $\omega=1 / \tau$. The Drude model cannot produce either real conductivity with a non-DC maximum or negative imaginary conductivity.

\subsubsection{Plasmon model}

The plasmon model is a direct generalization of the Drude model. This model is used to explain the $\mathrm{THz}$ conductivity of particles which are small compare to the wavelength of $\mathrm{THz}$ radiation [170]. If the particle size is small compared to the wavelength of $\mathrm{THz}$ radiation, charges cannot move outside the boundaries of the particle and result in plasmon resonance. The motion of electrons is described by a forced damped harmonic oscillator with a restoring force.

$$
\frac{d^{2} r}{d t^{2}}+\gamma \frac{d r}{d t}+\omega_{0}^{2} r=-\frac{e}{m} E(t)
$$

where $\omega_{0}$ is the resonant frequency. The conductivity associate with the plasmon model can be expressed as 


$$
\sigma(\omega)=\frac{N e^{2}}{m^{*}} \frac{i \omega}{\left(\omega^{2}-\omega_{0}^{2}-i \omega \gamma\right)}
$$

The plasmon model reduces to the Drude model for $\omega \gg \omega_{0}$. Figure 3.11 illustrates the conductivity spectra determined using the plasmon model. It can be observed that at low frequencies, the imaginary part of the conductivity is negative whereas the real part is always positive. The plasmon model is more appropriate for nanostructured semiconductors where strong excitonic or electron-hole populations can co-exist spatially.

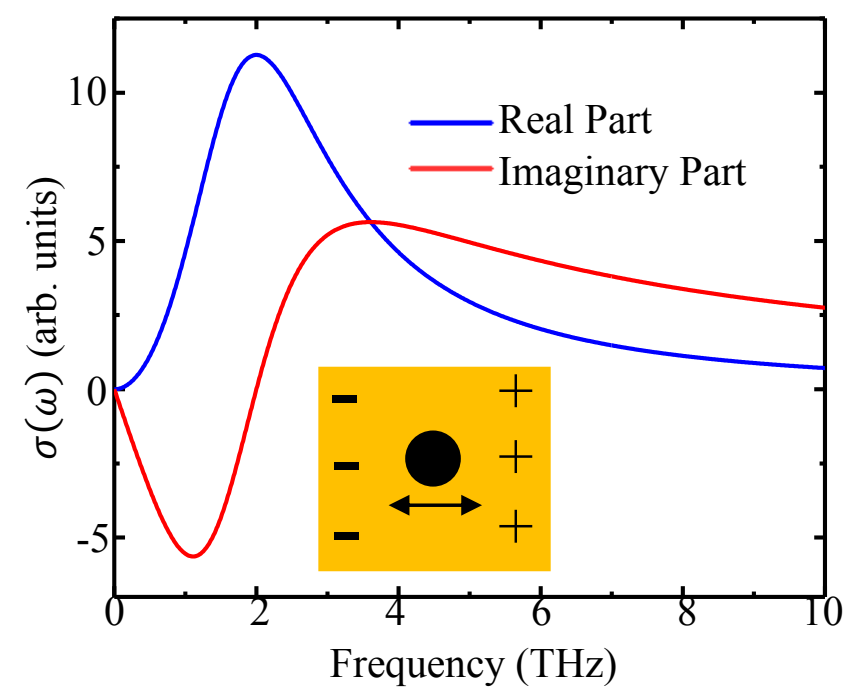

Figure 3.11. The real and imaginary part of the conductivity spectra determined using the plasmon model with $N=1 \times 10^{16} \mathrm{~cm}^{-3}, \gamma=2.5 \times 10^{12} \mathrm{~s}$ and $\omega_{0} / 2 \pi=2 \mathrm{THz}$

\subsubsection{Drude-Smith model}

Drude model cannot be used to describe several transport mechanisms such as the backscatter of electrons against the driving THz field. Also, it fails when the carrier scattering time is dependent on energy. The Drude-Smith model is an extension of the Drude model which is used to describe the carriers undergoing restricted motion [171,172]. In this model, carriers are allowed to scatter in a preferential direction whereas in the Drude model carrier scattering is isotropic. The expression for Drude-Smith conductivity is given by 


$$
\sigma(\omega)=\frac{N e^{2} \tau / m^{*}}{1-i \omega \tau}\left[1+\frac{c}{1-i \omega \tau}\right]
$$

where $N$ is the carrier concentration, $m^{*}$ is the effective mass, $\tau$ is the scattering time and $c$ is the backscattering coefficient which describes carrier confinement phenomenologically. The main assumption of this model is that backscattering persists for only one scattering event. This model is commonly used to describe the conductivity of nanostructured materials and systems in which long-range transport is not allowed by the extent of the sample or suppressed by disorder and defects. The parameter $c$, can take values between 0 and -1 .
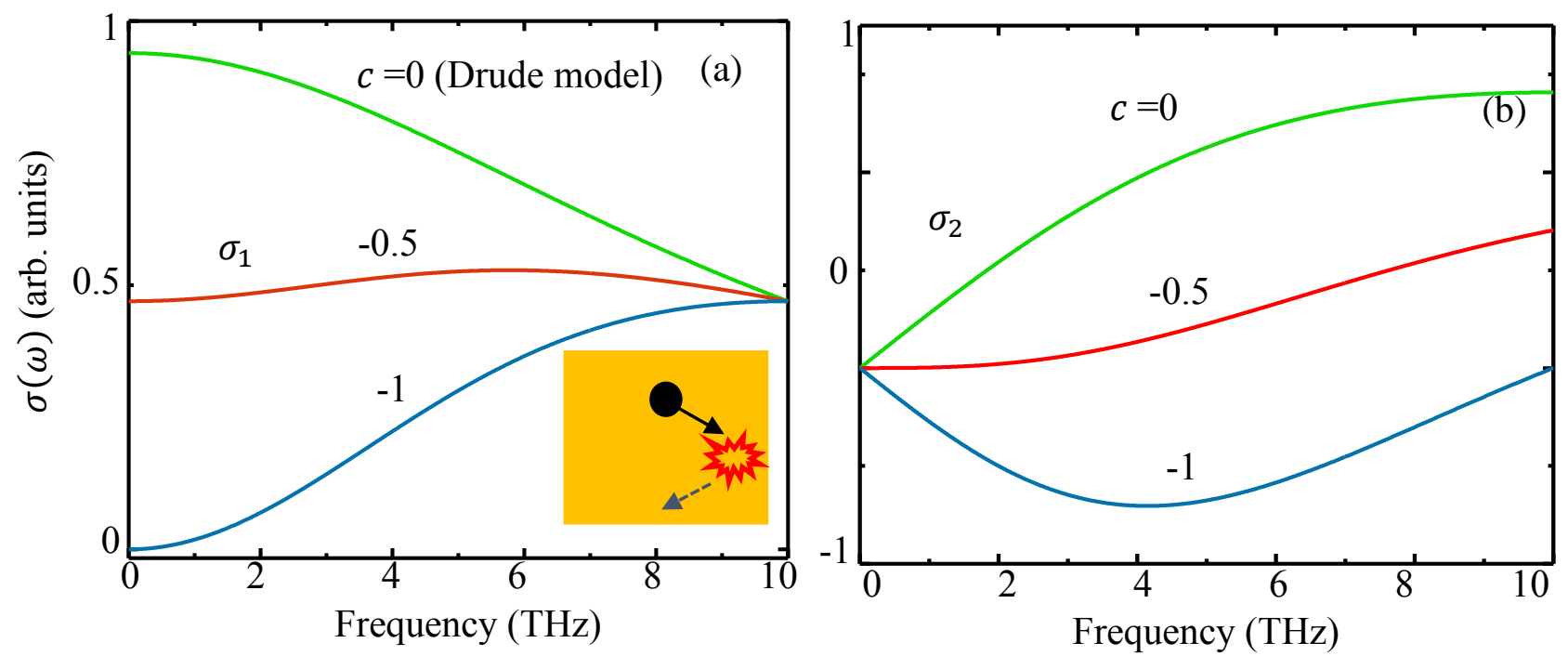

Figure 3.12. (a) Real and (b) imaginary part of conductivity derived using the Drude-Smith model for various values of $c$.

Figure 3.12 shows the real $\sigma_{1}$ and imaginary parts $\sigma_{2}$ of the Drude-Smith conduction response for different $c$ values. The Drude model can be reproduced for $c=0$. Higher negative $c$ values will suppress DC conductivity. For $c-=1$, the DC conductivity is completely suppressed. However, it has not been directly, concretely related to microscopic mechanisms or even evaluated in terms of tensor components to understand the restrictions. 


\subsubsection{Cole-Cole model}

Another commonly used phenomenological conductivity model is the Cole-Cole model given as

$$
\sigma(\omega)=\frac{N e^{2}}{m^{*}} \frac{\tau}{(1-i \omega \tau)^{1-\alpha}}
$$

where $\alpha$ is the Cole-Cole parameter which can take a value between 0 and 1 . The term $(1-\alpha)$ is known as the Cole-Cole model exponent, which describes the width of the relaxation time distribution. This model can be used to describe systems that have small deviations from the Drude model. Cole-Cole model cannot be used to describe negative imaginary conductivities.

\subsection{Conductivity Response of Chalcopyrite crystals}

In this section, the complex conductivity response of CGP, ZGP, and CGP crystal will be explored. The native conductivity, $\tilde{\sigma}(\omega)$, is determined from refractive index and extinction coefficients as described in section 3.2. The complex conductivity of CGP, ZGP, and CSP is measured from $4 \mathrm{~K}$ to $300 \mathrm{~K}$ over the frequency range of $0.4 \mathrm{THz}$ to $1.5 \mathrm{THz}$. For all three crystals real part of the conductivity $\sigma_{1}(\omega)$ is positive and increasing with frequency, whereas the imaginary part of the conductivity $\sigma_{2}(\omega)$ is negative and decreasing with frequency. Values of conductivity are low, but are consistent with Hall-effect measurements [57].

AC conductivity is related to carrier density $(N)$ and carrier-scattering time $(\tau)$, which in semiconductors is described by the Drude model or a variant of it [153]. Figure 3.13 (a) shows the conductivity fits using Drude model. According to this model, carrier transport is dominated by scattering events that randomize their momentum on a (sub) picosecond times. Hence, $\sigma_{1}(\omega)$ should increase with decreasing frequency in the THz range and the $\sigma_{2}(\omega)$ should also be positive. The measured $\sigma_{2}(\omega)$ could not fit using the Drude model since the experimental trends of $\sigma_{1}(\omega)$ and $\sigma_{2}(\omega)$ are inconsistent with the pure Drude model. Figure 3.13 (b) shows the conductivity fits using the surface plasmon model. However, they have a very poor fitting to the entire data range. Also, the surface plasmon model cannot apply to our samples since it uses to describe the $\mathrm{THz}$ conductivity of particles which are small compared to the wavelength of $\mathrm{THz}$ radiation. Figure 
3.13 (c) shows the conductivity fits using the Drude-Smith model.AC conductivities are fitted well by the Drude-Smith model. Figure 3.13 (d) shows the conductivity fits using the Cole-Cole model. However, this model cannot produce a negative $\sigma_{2}(\omega)$.
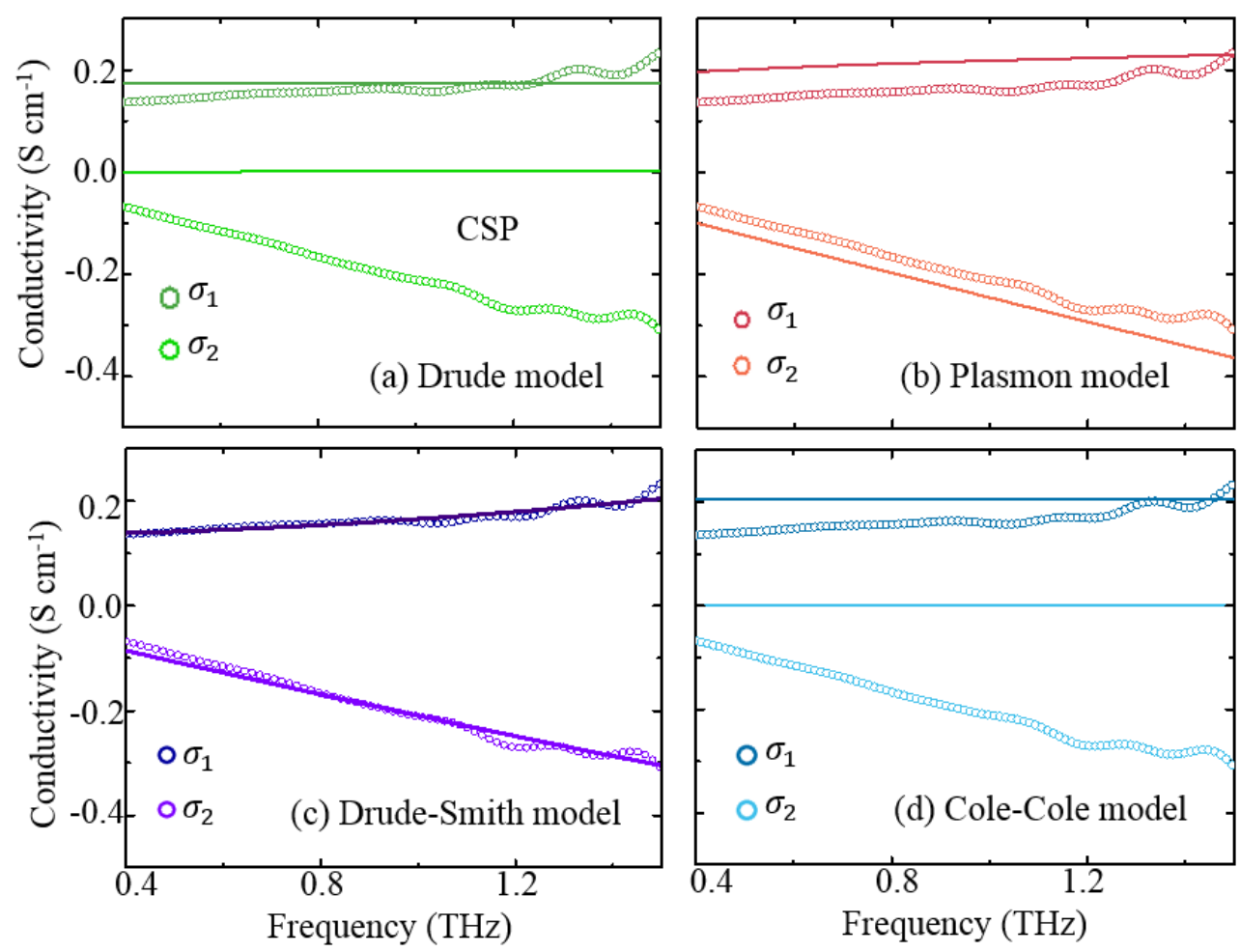

Figure 3.13. Real $\left(\sigma_{1}\right)$ and imaginary parts $\left(\sigma_{2}\right)$ of AC conductivity for CSP crystal fitted using (a) Drude model (b) surface plasmon model (c) Drude-Smith model (d) Cole-Cole model.

Figure 3.14 shows $\sigma_{1}(\omega)$ and imaginary $\sigma_{2}(\omega)$ parts of the conductivity for (a) CGP, (b) ZGP, and (c) CSP both at $4 \mathrm{~K}$ and $300 \mathrm{~K}$, shown as open squares and circles respectively. The Drude-smith model is used to describe the observed conductivity response. The experimental AC conduction results are fit well by the Drude-Smith model, as seen by the solid lines that overlay the data points. The negative imaginary conductivity observed in all samples is attributed to the backscatter of electrons against the driving THz fields. It can be observed that there is an increase in the complex conductivity at $300 \mathrm{~K}$ compared to $4 \mathrm{~K}$ for all three crystals. The effective masses 
used for the fittings are $m^{*}=0.088 m_{0}, 0.108 m_{0}$ and $0.124 m_{0}$ for CGP, ZGP [33], and CSP [173].
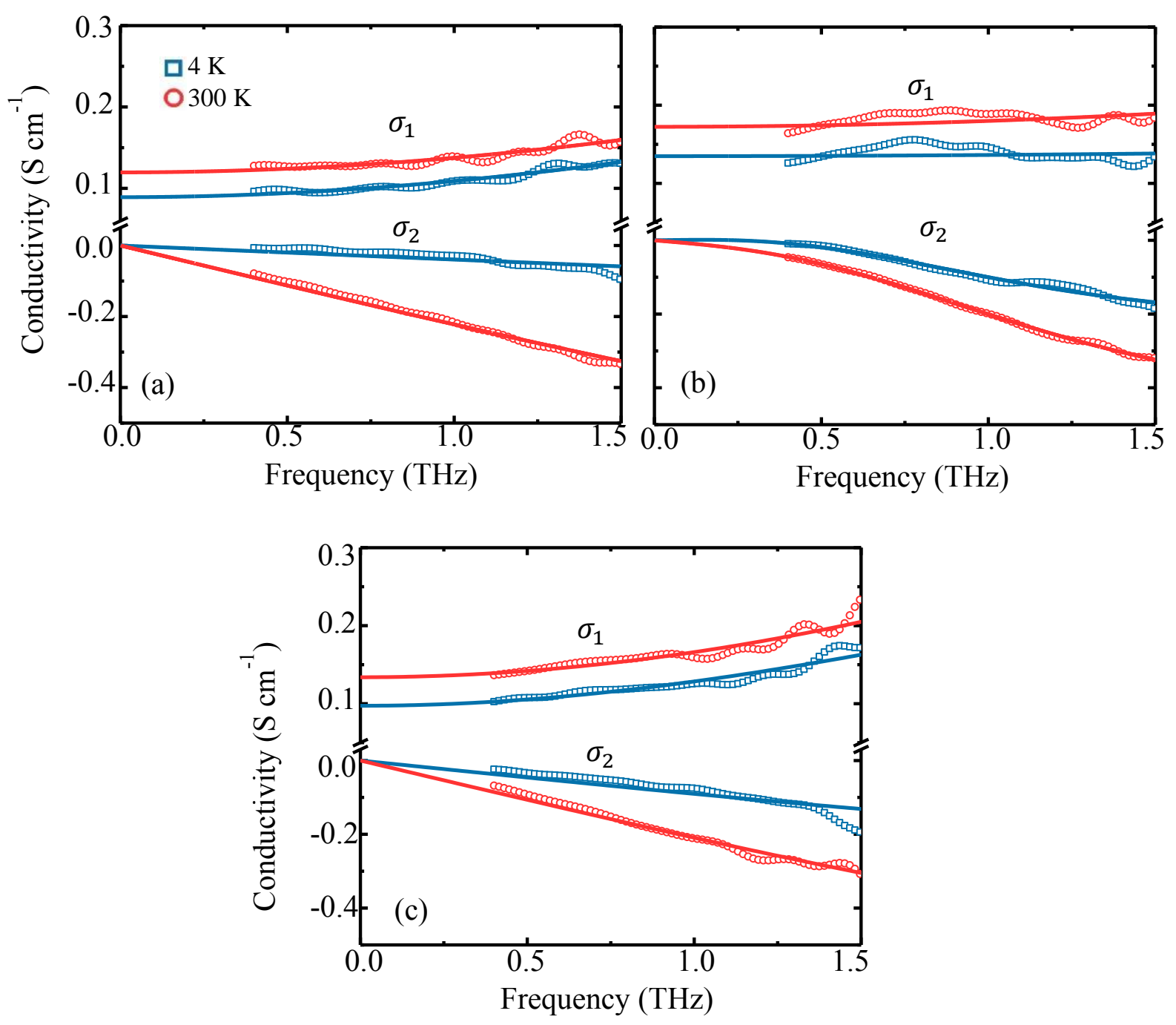

Figure.3.14. Real $\left(\sigma_{1}\right)$ and imaginary parts $\left(\sigma_{2}\right)$ of AC conductivity for (a) CGP (b) ZGP and (c) CSP crystals with lattice temperatures of $4 \mathrm{~K}$ and $300 \mathrm{~K}$, fit by a Drude-Smith conduction model. 


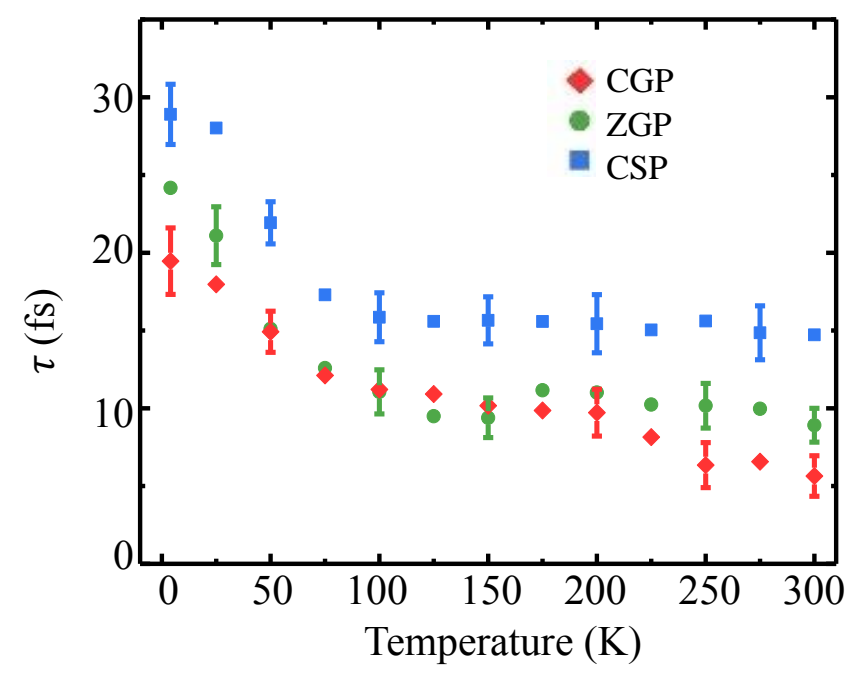

Figure 3.15. Scattering time for all three crystals as a function of temperature.

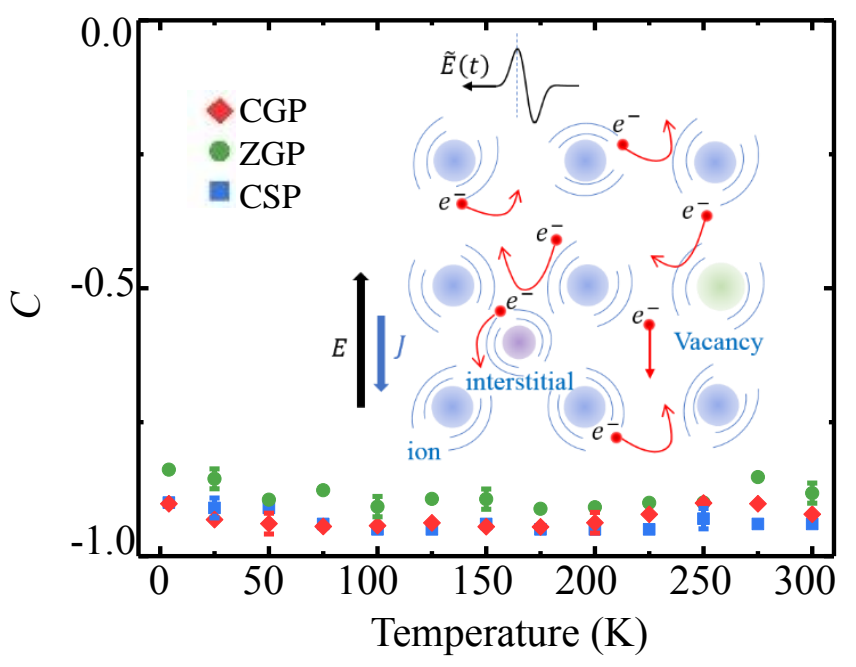

Figure 3.16. Backscattering coefficient for all three crystal as a function of temperature

Figure 3.15 shows the extracted scattering time as a function of lattice temperature for all three crystals. It can be seen that the scattering time decreases with increasing lattice temperature. Figure 3.16 shows the extracted backscattering coefficient, $c$, from the Drude-Smith model as a function of temperature for all three crystals. In all three cases, $c$ is non-negligible and reasonably independent of temperature, which indicates strong carrier backscattering associated with the 
crystal structure and not carrier occupancy. In nanostructures, the carrier backscatter is easily justified by the structure itself [172]. By contrast, the remaining sources of backscatter in highquality bulk semiconductors with low polycrystallinity [174] are defects, dislocation, and vacancies, which can be associated with electronic impurities (i.e., unintentional dopants) [104] and as has been observed in lightly-doped GaAs and Si samples [142]. In the next sections, the temperature dependence of the extracted $N$ from the Drude-Smith model will be discussed.

\subsection{Temperature-Dependent Carrier Density}

Defects and dopants have a significant influence on the properties of semiconductors such as optical properties, electronic properties, resistivity, lifetime, and carrier mobility. There are two major classes of defects: point defects and extended defects. Point defects involve either a single atomic site or few nearest neighbors. Grain boundaries, dislocations, and stacking faults are some examples of extended defects. Figure 3.17 shows various point defects in $\mathrm{ZnGeP}_{2}$ crystal.

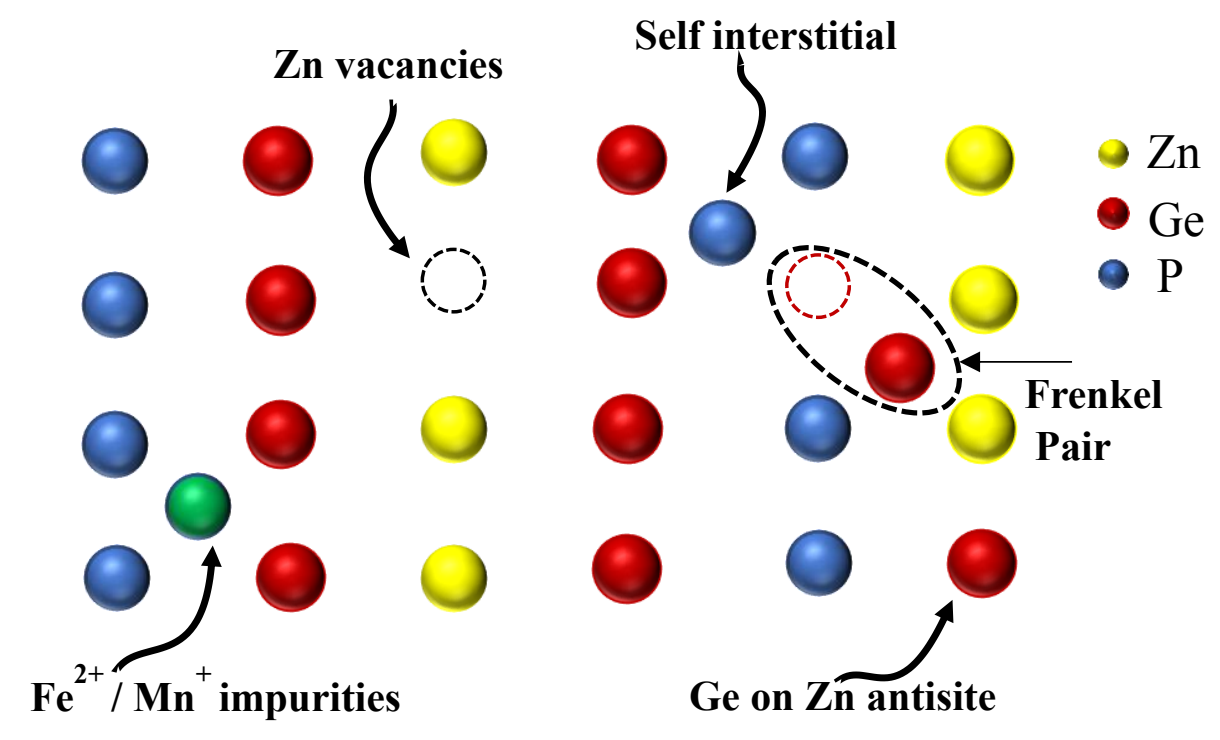

Figure 3.17. A schematic illustration of different point defects in $\mathrm{ZnGeP}_{2}$ crystal 
Defects introduce an energy state in the forbidden gap of the material hence they considerably modify the electrical conductivity of the material. Defects can be classified as shallow and deep levels according to the position of the energy state. The energy levels of the shallow defects are few millielectron volts from the band edges consequently these levels are thermally ionized at room temperature. Impurities present in semiconductors usually introduce these shallow levels and provide free carriers. Hence shallow impurities dramatically change the type of electrical conductivity, mobility, and resistivity of a semiconductor. The energy levels of deep impurities typically lie deeper in the band gap and the wave function of the deep levels is highly localized. Deep levels have higher ionization energies compare to shallow impurities hence their contribution to the free-carrier concentration in the sample is negligible. Deep levels in the band gap are frequently known as traps or recombination centers. They can control the lifetime of carriers through recombination mechanisms.

A shallow impurity/dopant is similar to a hydrogen atom with a positive nucleus binding an electron. Here, two major modifications should be considered when applying the hydrogen model to shallow dopants. First, the electron mass is renormalized (effective mass) by the periodic potential. This effective mass is smaller than the free electron mass. Secondly, Coulomb interaction is screened by the dielectric constant of the material. With these modifications, the binding energy, $E_{D}^{b}$, of the electron to the shallow dopant can be expressed as follows [149]

$$
E_{D}^{b}=\frac{m_{e}^{*} e^{4}}{2\left(4 \pi \epsilon_{0} \epsilon_{r} \hbar\right)^{2}}
$$

where $m_{e}^{*}$ is the effective mass $\epsilon_{r}$ is the static relative dielectric constant and $\hbar$ is the reduced Planck constant. The ground state binding energy of a dopant is significantly reduced by about $10^{-3}$ compare to the hydrogen atom. The radius of the orbit is given by the following equation

$$
a_{D}=\frac{m_{0}}{m_{e}^{*}} \epsilon_{r} a_{B}
$$

where $a_{B}=0.053 \mathrm{~nm}$ is the hydrogen Bohr radius. The radius of the orbit is much larger compared to the hydrogen atom.

Dopants change the free-carrier occupancy as a function of temperature; hence, $N(T)$ reveals information about the scattering sources. Occupancy is described with a Fermi-Dirac 
model [149], including a term that aggregates all dopants with a dopant concentration, $N_{D}$, and an average dopant binding energy, $E_{D}^{b}$. The model is given as

$$
N(T)=\frac{2 N_{D}}{1+\sqrt{1+4 \hat{g}\left(N_{D} / N_{C}\right) \exp \left(E_{D}^{b} / k T\right)}},
$$

where $N c=2\left(m_{e} k T / 2 \pi \hbar^{2}\right)^{\frac{3}{2}}, \hat{g}=g_{1} / g_{0}, g_{1}$ is the degeneracy of the dopant charged with one electron, $g_{0}$ is the degeneracy of the ionized dopant, $m_{e}$ mass of the electron and $\hbar$ is the reduced Planck constant.

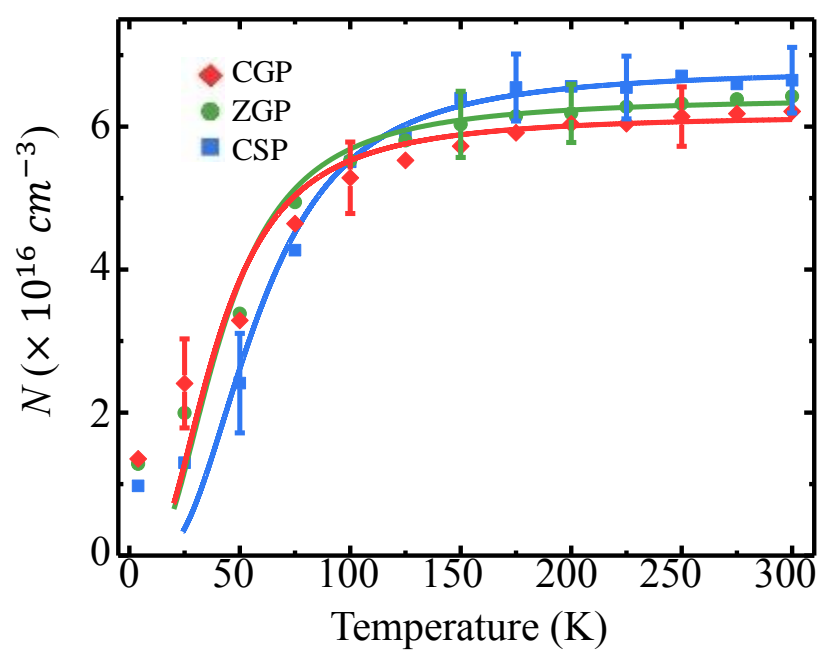

Figure 3.18. Temperature-dependent carrier density $(\mathrm{N})$ fit with a Fermi-Dirac model.

Figure 3.18 shows the extracted $N(T)$ from the Drude-Smith model as a function of temperature for all three crystals. $N(T)$ increases with temperature up to approximately $100 \mathrm{~K}-$ $150 \mathrm{~K}$ for the various crystals, saturating above that temperature. This trend is described by the increasing ionization of dopants up to $N_{D}$ [149] and is fit well with the Fermi-Dirac model. Parameters from the Fermi-Dirac model are shown in Table 4. The values of dopant concentration are reasonable and consistent with high-quality crystals. 
Table 4. Extracted Parameters from the Fermi-Dirac Model.

\begin{tabular}{llc}
\hline Sample & $N_{D}\left(\times 10^{16} \mathrm{~cm}^{-3}\right)$ & $E_{D}^{B}(\mathrm{meV})$ \\
\hline CSP & $6.85 \pm 0.41$ & $13.13 \pm 0.69$ \\
ZGP & $6.42 \pm 0.32$ & $8.69 \pm 0.43$ \\
CGP & $6.18 \pm 0.31$ & $8.19 \pm 0.41$ \\
\hline
\end{tabular}

The average dopant binding energy results from a variety of defects and vacancies and depends on the material. Electron paramagnetic measurements (EPR) of CSP crystals [64,175] have identified silicon, cadmium, and phosphorus vacancies, and silicon-on-cadmium anti-site defects. Silicon and cadmium vacancies act as acceptors, whereas phosphorous vacancies and silicon-on-cadmium anti-site defects act as donors, which partly compensate one another and will exhibit different degeneracy and temperature dependencies. EPR has also observed unintentionally $\mathrm{Mn}^{+}, \mathrm{Fe}^{+}, \mathrm{Fe}^{2+}$ and $\mathrm{Fe}^{3+}$ ions in addition to the main native defects formed during growth. Silicon vacancy dominant low-temperature EPR signatures and silicon-on-cadmium anti-site defects or phosphorus vacancies are more prevalent at higher temperatures. Similarly, ZGP crystals exhibit zinc, phosphorus, and germanium vacancies, and germanium-on-zinc anti-site defects [65,176]. Zinc vacancy dominates the low-temperature EPR response, supplanted by germanium-on-zinc anti-site or phosphorus vacancy at higher temperatures. Defect data for CGP was not found but is presumed to be similar to ZGP and CSP. The temperature-dependent defect response in EPR indicated that scattering must also have a temperature dependence arising from a range of mechanisms, especially since some of the native defects are donors and others acceptors $[64,65,175]$. 


\subsection{Microscopic Scattering Mechanisms}

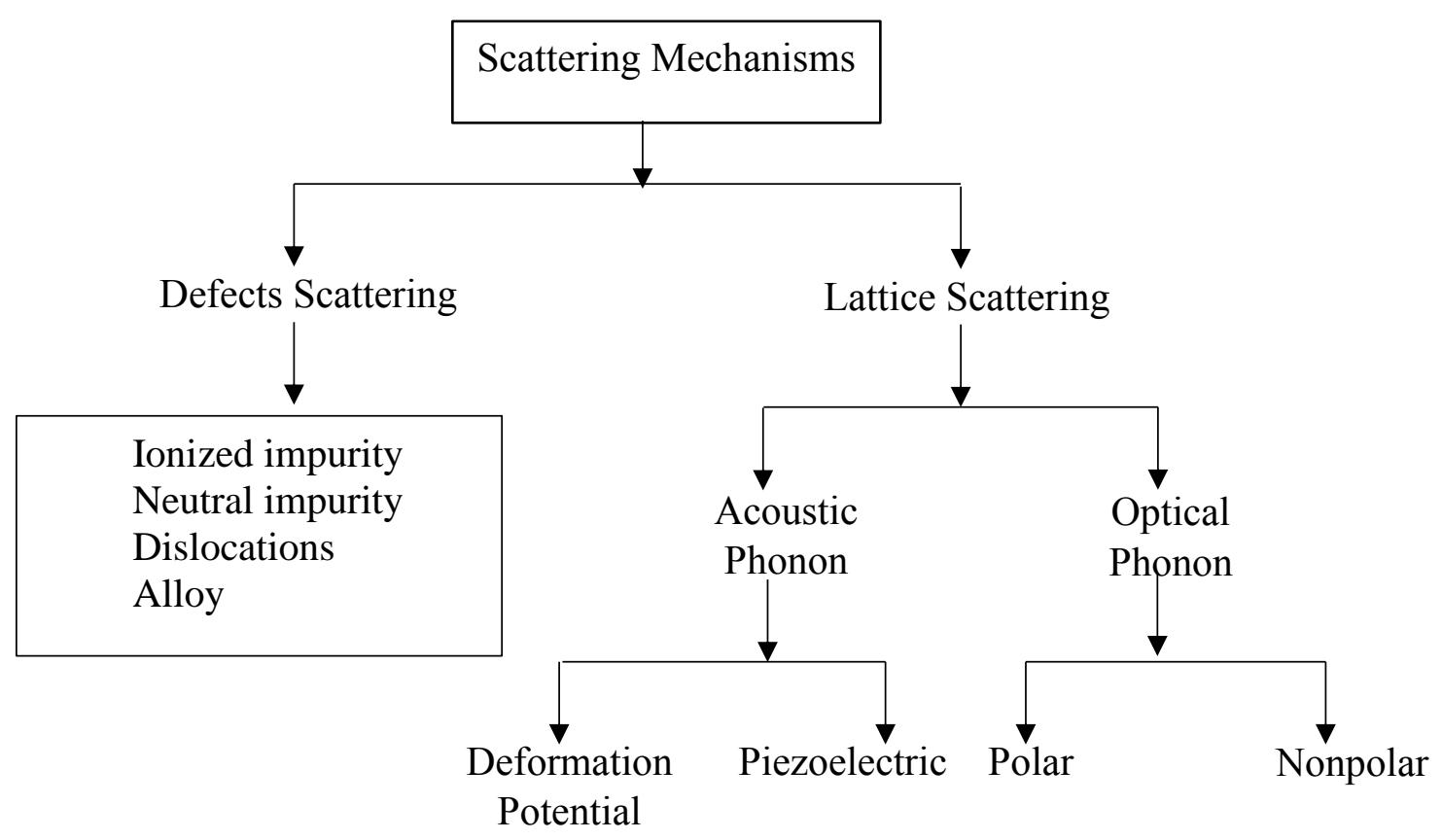

Figure 3.19. Types of scattering mechanisms in a typical semiconductor

Relaxation times and carrier mobilities are crucial parameters for semiconductors and their use in optoelectronic devices. In these semiconductors, carrier transform is governed by the scattering mechanisms, which are a combination of electronic interaction with the driving field, the lattice, and other electrons. Each contribution has a temperature dependence due to varying carrier concentration, phonon density, lattice expansion, and related mechanical properties, and degree of defect ionization. These different types of scattering mechanisms strongly influence the carrier mobility in a semiconductor. Figure 3.19 summarizes the various types of scattering mechanisms.

The extracted backscattering coefficient and momentum scattering time from Figures 3.15 and 3.16 can be related to the carrier mobility to characterizes the transport as is done in Halleffect measurements. Since the back-scattering coefficient is reasonably independent of temperature, the mobility can be expressed as 


$$
\mu(T)=\left(\frac{e}{m^{*}}\right) \tau(T)
$$

where $e$ is the electron charge and $m^{*}$ is the reduced mass. The mobility consists of a variety of scattering mechanisms. If these various scattering mechanisms are independent of each other, the total mobility can be obtained using the Matthiessen rule as follows

$$
\frac{1}{\mu}=\sum_{i} \frac{1}{\mu_{i}}
$$

where the $i^{\text {th }}$ component is described below. Various components are required to balance the changes in the lattice vibrations and electronic occupation with temperature. In this section, various important scattering mechanisms such as ionized impurity scattering, dislocation scattering, acoustical phonon scattering, and optical phonon scattering will be discussed.

\subsubsection{Ionized-Impurity Scattering}

Impurities and defects present in the sample influence the motion of electrons and leads to scattering. Impurities can be either neutral or charged. Ionized impurity scattering from the atomic cores is a variant of Rutherford scattering with a Coulomb potential in the Thomas-Fermi screening limit. This scattering mechanism is very significant in doped semiconductors. Ionized impurity scattering is dominant at low temperatures since phonon scattering is minimal. The mobility due to ionized impurity scattering can be described as $[177,178]$

$$
\mu_{i o n}=\frac{2^{7 / 2}\left(4 \pi \varepsilon_{0} \varepsilon_{D C}\right)^{2} k T^{3 / 2}}{\pi^{3 / 2} Z^{2} e^{3} \sqrt{m^{*}} N_{i o n} \ln \left[1+\left(\frac{3 \varepsilon_{0} \varepsilon_{D C} k T}{2 Z e^{2} N_{i o n}^{1 / 3}}\right)^{2}\right]}
$$

where $\varepsilon_{D C}$ is the DC dielectric constant, $Z$ is the charge of ions and $N_{i o n}$ is the ionized impurity concentration. 


\subsubsection{Deformation-Potential Scattering}

Electrons can interact with various types of phonons by absorbing or emitting a phonon. Scattering of electrons by longitudinal acoustic phonon is a major scattering mechanism in lightly doped semiconductors. This scattering mechanism is considered as an elastic scattering since the change in electron energy is insignificant compared to the average electron energy. There are two different ways that acoustic phonon cause scattering in a semiconductor:deformation potential scattering and piezoelectric scattering. The acoustic longitudinal phonons induce displacement of the atoms from their lattice site in a semiconductor. This will induce a local fluctuation of the band gap on an atomic scale and results in a potential known as deformation potential. The deformation potential is defined as the change of band gap energy per unit strain due to acoustic phonons. In 1950 Bardeen and Shockley derived the mobility due to deformation potential scattering and given as $[177,178]$

$$
\mu_{\text {def }}=\frac{2 \sqrt{2 \pi} e \hbar^{4} c_{l}}{3 m^{* 5 / 2} E_{d e f}^{2}}\left(\frac{1}{k T}\right)^{3 / 2},
$$

where $c_{l}$ is the elastic constant for longitudinal deformation and $E_{d e f}$ is the deformation potential.

\subsubsection{Piezoelectric Scattering}

Piezoelectric scattering is another type of acoustic-phonon scattering. This is a dominant scattering mechanism in polar crystals such as semiconductors with zincblende and wurtzite crystal structures. Due to the lack of inversion symmetry in these semiconductors, longitudinal acoustic phonons create a strain-induced macroscopic electric field, which leads to piezoelectric scattering. The mobility associate with piezoelectric scattering is given as $[177,178]$

$$
\mu_{p z}=\frac{16 \sqrt{2 \pi} \hbar^{2} \varepsilon_{0} \varepsilon_{D C}}{3 m^{* 3 / 2} e K^{2}}\left(\frac{1}{k T}\right)^{\frac{1}{2}}
$$

where $K=\left(e_{p}^{2} / c_{l}\right) /\left(\varepsilon_{0} \varepsilon_{D C}+e_{p}^{2} / c_{l}\right)$ with $e_{p}$ as the piezoelectric coefficient. 


\subsubsection{Optical-Polar Scattering}

Electron scattering by polar optical phonon is a dominant scattering mechanism in ionic crystals such as II-VI and III-V semiconductors. Since optical phonons induce an atomic polarization in polar semiconductors, electrons are scattered through the interaction of the Coulomb field. This scattering mechanism is generally an inelastic process since, at room temperature, the energy of the optical phonon is comparable to that of the electron mean energy. Optical polar phonon scattering is the major mechanisms limiting the mobility at relatively high temperatures for III-V semiconductors. The mobility associate with optical polar scattering is given as $[177,178]$

$$
\mu_{p o l}=\frac{e}{2 m^{*} \alpha \omega_{0}} \exp \left(\frac{\theta_{D}}{T}\right)
$$

where $\alpha$ is the polar constant, $\omega_{0}$ is longitudinal optical phonon frequency and $\theta_{D}$ is the Debye temperature.

\subsubsection{Scattering from Dislocations}

Dislocation scattering arises from charge centers created by defect sites. Dislocations in a crystal induce strain fields which will result in deformation potential. The scattering from dislocations is considerable if the density of dislocations is greater than $10^{8} \mathrm{~cm}^{-2}$. Debye length $\lambda_{D}=\left(\varepsilon_{0} k T / e^{2} N\right)^{1 / 2}$ describing the screening and $\xi_{\perp}=\left(2 k_{\perp} \lambda_{D}\right)^{2}$ describes the transverse component of the kinetic energy of the carriers. The mobility associates with dislocation scattering is given by $[177,178]$

$$
\mu_{d i s}=\frac{\hbar^{3} \varepsilon_{0}^{3} c^{2} e N^{2}}{e^{3} f^{2} m^{* 2} N_{d i s} k^{2} T^{2}}\left(1+\frac{4 k_{\perp} \varepsilon_{0} k T}{e^{2} N}\right)^{3 / 2}
$$

where $f$ is the fraction of filled traps, $N_{\text {dis }}$ the dislocation density, $N$ is the carrier concentration and $k_{\perp}$ is the projection of the electron wave vector in the direction of the dislocation. 

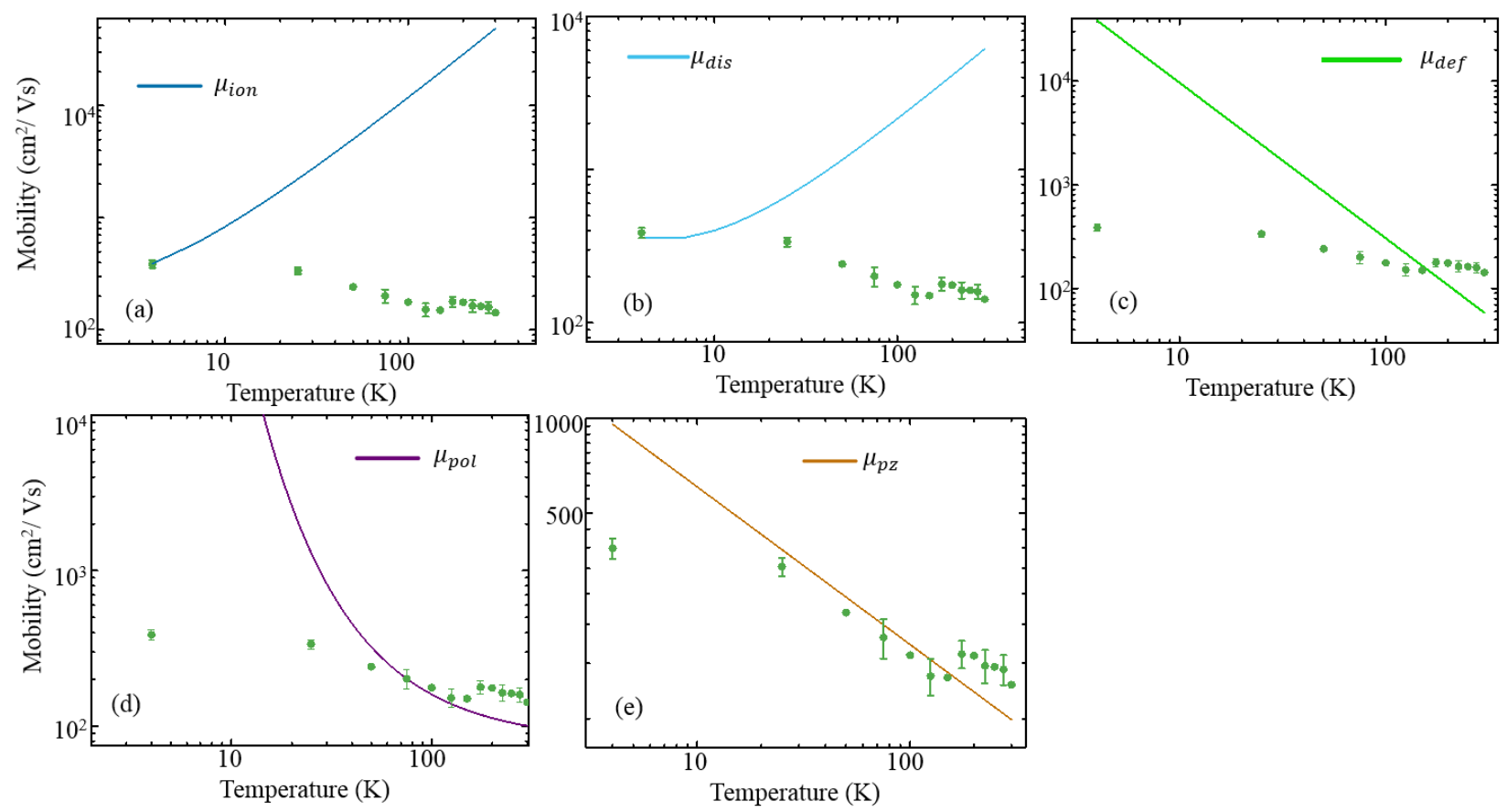

Figure 3.20. Temperature-dependent mobility fit with (a) ionized impurity scattering (b) dislocation scattering (c) deformation potential (d) optical polar scattering mechanism (e) piezoelectric scattering mechanism for ZGP crystal.

The carrier scattering time extracted from the Drude-Smith model is converted into mobility using equation 3.30. We tried to fit the experimental mobility using individual scattering mechanisms. However, they have a very poor fitting to the entire data range. Figure 3.20 shows mobility fits with individual components for the ZGP sample. It can be seen that the optical polar scattering fits the higher temperature range. However, Debye temperature and polar constant values extracted from the fitting strongly deviates from the literature values. The piezoelectric scattering has the best fit for the data at higher temperatures. To fit the low-temperature regime, ionized impurity scattering, or dislocation scattering should be included. Although a single scattering mechanism mainly dominates at a specific temperature, other scattering mechanisms should be included to fit the entire temperature range.

The data are fit with a model that incorporates several scattering mechanisms as described in equation 3.31. Figure 3.21 shows $\mu(T)$ in the lower panels for the three crystals to decrease with increasing temperature. The upper panel of each figure shows the individual contribution to total 
mobility. The scattering model is applied to all three crystals and the modeling parameters are shown in Table 5.

Literature value of $\theta_{D}$ are used [179]. $c_{l}$ values are in good agreement with literature values [39,60,180] and values of $\alpha$ and $\omega_{0}$ are consistent with calculations [181]. Values of $N_{i o n}$ are in reasonable agreement to estimated $N_{D}$ from the Fermi-Dirac model. Finally, to the best of the authors knowledge, deformation potential and piezoelectric coefficient of CGP, ZGP, and CGP are reported for the first time. Overall, the modeling shows that in CGP and CSP the lowtemperature mobility is dominated by $\mu_{d i s}$, then subsequently by $\mu_{p z}$ and $\mu_{p o l}$ as the temperature increases. For ZGP, $\mu_{i o n}$ dominate the low-temperature response with $\mu_{p z}$ and $\mu_{p o l}$ dominating at higher temperatures. Finally, it is clear that CGP and CSP exhibit more dislocations than the ZGP sample.

Table 5. Extracted parameters from mobility fitting for three crystals

\begin{tabular}{llll}
\hline Parameter & CGP & ZGP & CSP \\
\hline$c_{l}\left(\times 10^{11} \mathrm{Nm}^{-2}\right)$ & 1.84 & 1.12 & 1.64 \\
$E_{\text {ac.def }}$ & 8.65 & 8.10 & 7.96 \\
$e_{p}\left(\mathrm{Cm}^{-2}\right)$ & -3.97 & -4.85 & -5.06 \\
$\theta_{D}(\mathrm{~K})$ & 319 & 391 & 376 \\
$\omega_{o} / 2 \pi(\mathrm{THz})$ & 6.65 & 8.15 & 7.83 \\
$\alpha$ & 0.05 & 0.03 & 0.07 \\
$N_{\text {ion }}\left(\times 10^{15} \mathrm{~cm}^{-3}\right)$ & 5.36 & 9.74 & 3.57 \\
$N_{\text {dis }}\left(\times 10^{10} \mathrm{~cm}^{-2}\right)$ & 2.74 & 0.55 & 1.03 \\
\hline
\end{tabular}



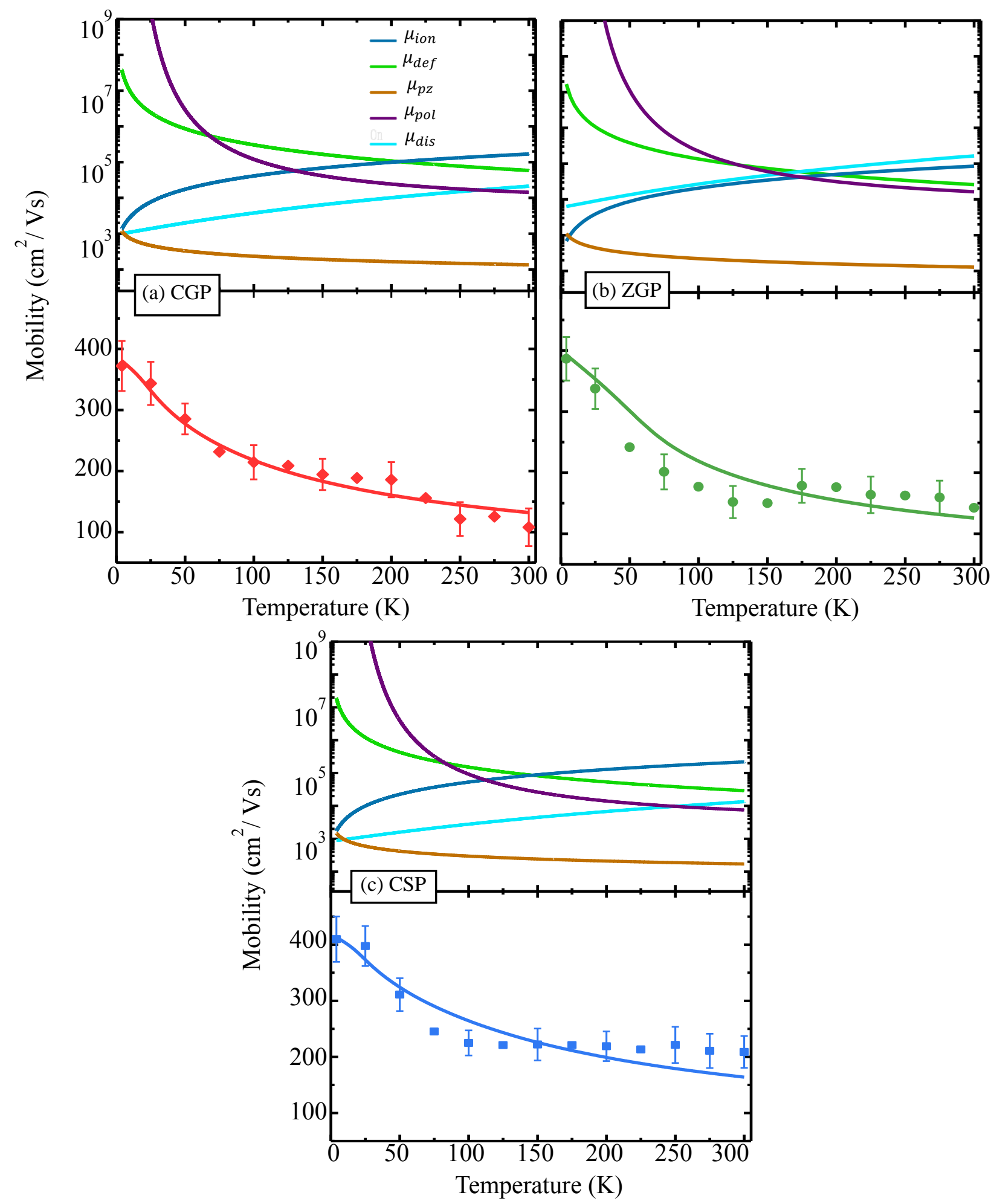

Figure 3.21. Temperature-dependent mobility fit with a multi-component scattering model for (a) CGP, (b) ZGP and (c) CSP. Solid lines represent the theoretical contributions from different scattering mechanisms. Figure modified from Sooriyagoda et al. [158] 


\subsection{Chapter Summary}

Temperature-dependent THz-TDS characterizes both the complex refractive index and AC conductivity and is applied to model semiconductors CGP, ZGP, and CSP. This approach has been used to perform spectroscopic analysis that is related to microscopic carrier transport including carrier-lattice interactions that modify the transport.

First, the temperature-dependent $\mathrm{THz}$ refractive index yields the average phonon frequency and the electron-phonon coupling constant. Second, $\mathrm{THz}$ absorption confirms the center frequencies of the lowest-lying optical phonon modes for each temperature. Third, temperaturedependent AC conductivity clarifies the scattering/backscattering regime, carrier concentration from dopant, and their average binding energy. Finally, temperature-dependent mobility is described by a combination of polar optical phonons, acoustic phonons, deformation potential, ionized impurity, and dislocation scattering.

This study reported the deformation potential and piezoelectric coefficient of CGP, ZGP, and CGP for the first time. Moreover, this work shows links between non-contact THz-TDS and Hall-effect measurements. Applied to nonlinear and optoelectronic materials, this spectroscopic characterization is of great use to large-area crystal growth and device fabrication by providing feedback on the electronic properties through non-invasive measurements. 


\section{Chapter 4: Non-Equilibrium Carrier Dynamics}

\subsection{Introduction}

To develop and optimize semiconductor devices such as lasers, photodetectors, photovoltaic devices, and light-emitting diodes, it is important to understand the various dynamical processes in semiconductors such as carrier generation, recombination mechanisms, excited-state lifetime, mobilities, and non-equilibrium transport mechanisms. As explained in chapter 02, TimeResolved THz Spectroscopy (TRTS ) is a powerful technique to measure charge transfer and recombination kinetics, as well as far-infrared AC photoconductivity of a material with subpicosecond to nanosecond temporal resolution in a non-destructive manner $[105,107,182]$. Here, a femtosecond optical pump pulse is used to excite conduction-band electrons and valence-band holes in a sample. Subsequently, a broadband terahertz $(\mathrm{THz})$ pulse is used to probe the changes induced in conductivity by the pump pulse. Since TRTS is a non-contact technique, it does not need ohmic contact and attaching wires or fabrications to the sample. This technique is widely used to probe the photoconductivity of semiconductors since the terahertz frequency range is on the order of the typical carrier-scattering rates of $10^{12}$ to $10^{14} \mathrm{~s}^{-1}[105,183]$. Instantaneous carrier density and mobility at a single time in a transient measurement can be obtained by fitting the measured AC photoconductivity spectra with the Drude or modified Drude conductivity model.

In this chapter ultrafast carrier dynamics and AC photoconductivity of CGP, ZGP, and CSP chalcopyrite crystals are investigated using TRTS. Optical pump-THz probe measurements have been carried out as a function of temperature and pump fluence. It is found that the $\mathrm{THz}$ transients decrease with increasing temperature. Also, the $\mathrm{THz}$ transients increase with increasing pump fluence. Differential $\mathrm{THz}$ transients have been fitted with a multiexponential function. It reveals a two-component exponential relaxation. Higher excitation fluences result in a slower relaxation due to saturation of available trap states. The inversion analysis technique is applied to the experimental transients to recapture the rate equation from the measured transient solution to the rate equation. From this rate equation, the carrier density-dependent mechanism can be inferred without prejudging the dominant mechanisms in a given carrier excitation regime. 
Frequency-dependent photoinduced conductivity in bulk chalcopyrite crystals has been measured as a function of temperature and pump-probe delay time. Carrier density, scattering time and backscattering coefficients are extracted by fitting complex AC photoconductivity with the Drude-Smith model. Carrier density decreases with pump-probe delay time since photoexcited carriers recombine or trap. Carrier mobility decreases with increasing temperature due to increased scattering from phonons and thermally excited carriers at higher temperatures. Increased carriercarrier interactions at higher carrier densities will lead to lower mobility.

\subsection{Charge-Carrier Recombination}

(a)

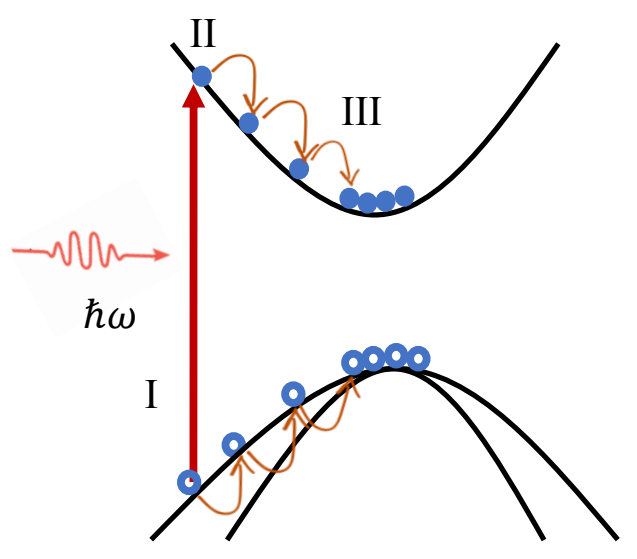

(b)

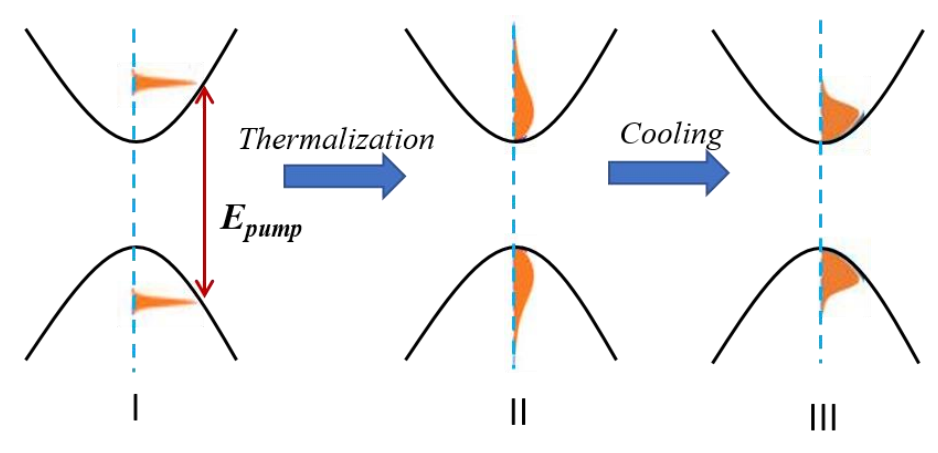

Figure 4.1. Schematic of the generation, thermalization and cooling (a) show these mechanisms from a band structure point of view (b) temporal development of the carrier population. (I) the above bandgap excitation and non-thermal carrier distribution. (II) thermalization of the excited carriers via carrier-carrier scattering. (III) cooling of carriers via carrier-phonon scattering.

When a semiconductor is illuminated with a laser pulse with a photon energy, $\hbar \omega$, that is larger than the bandgap energy, $E_{g}$, electrons-holes pairs are formed and create a non-equilibrium condition. Following the photoexcitation, carriers are in non-thermal distribution with a population density that resembles the pulse profile (amplitude and bandwidth) convolved with the oscillator strength over that excitation energies. The latter term is determined by the unoccupied density of 
states (in energy and momentum space) to give the dispersion of the optical matrix elements in Fermi's golden rule [146].

Typically, within 10 to 100 fs after excitation, carriers thermalize via carrier-carrier and optical-phonon scattering processes, resulting in a hot Fermi-Dirac distribution with a quasiequilibrium carrier temperature, $T_{C}$, over the lattice temperature, $T$. As time evolves further, the hot carriers lose their excess kinetic energy and cool intraband to the lattice temperature within picoseconds via phonon emission [79,80]. Finally, carriers undergo recombination. Figure 4.1 shows an example of the main processes taking place in a direct-gap semiconductor after photoexcitation.

(a)

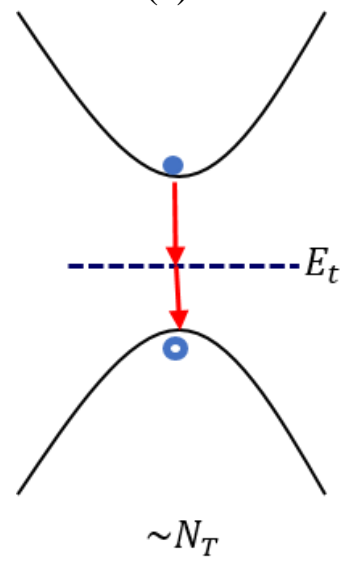

(b)

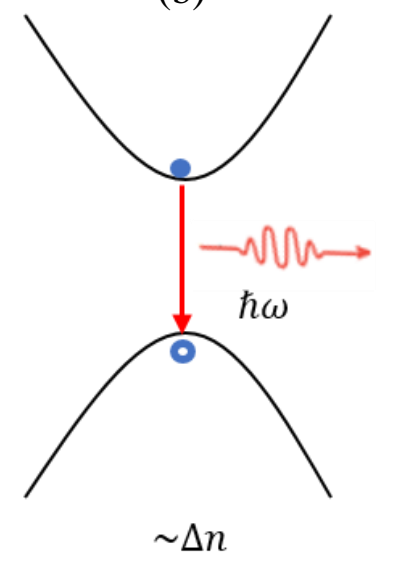

(c)

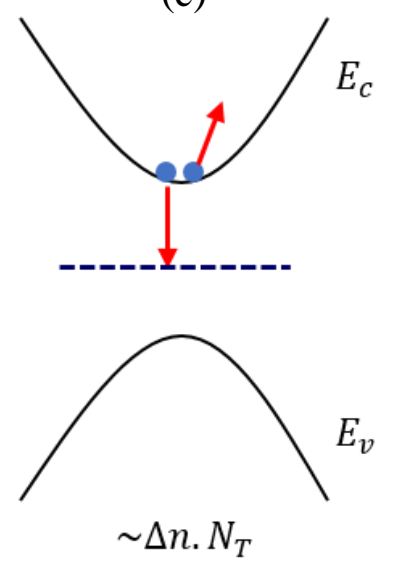

(d)

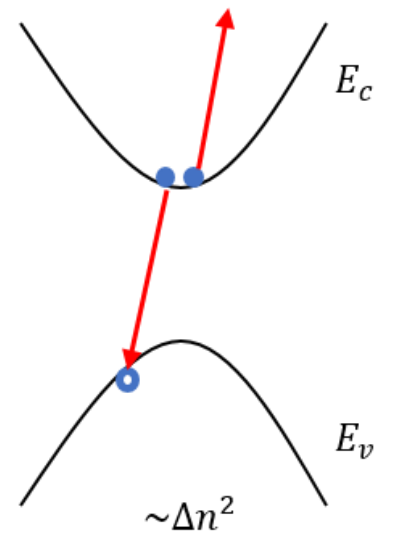

Figure 4.2. Main recombination mechanisms in semiconductors (a) SRH recombination (b). Radiative recombination (c) Trap-assisted Auger recombination and (d) Auger recombination

Figure 4.2 shows the various common recombination mechanisms seen in direct-gap semiconductors. First, in Shockley-Read-Hall (SRH) recombination, electrons recombine with holes through trap levels and energy is released as phonons. This mechanism is introduced by Hall, Shockley, and Read in 1952 [184,185]. SRH recombination mechanism is most effective when energy levels of the trap sites close to the middle of the band gap. The rate of this recombination is given as

$$
R_{S R H}=\frac{\left(n p-n_{i}^{2}\right)}{\tau_{p}\left(n_{0}+n_{1}+\Delta n\right)+\tau_{n}\left(p_{0}+p_{1}+\Delta p\right)}
$$


where $n$ and $p$ are total electron and hole density respectively, $n_{i}^{2}=n_{0} p_{0}, n_{i}$ is the electron concentration in intrinsic semiconductors at the equilibrium, $n_{o}$ and $p_{o}$ are the equilibrium carrier concentrations of electrons and holes respectively. $\Delta n$ and $\Delta p$ are the excess electron and hole concentration, $\tau_{p}$ and $\tau_{n}$ are hole and electron lifetimes defined as

$$
\tau_{p}=1 / N_{t} v_{p} \sigma_{p} \quad \tau_{n}=1 / N_{t} v_{n} \sigma_{n}
$$

with

$$
n_{1}=n_{i} \exp \left(\left(E_{t}-E_{F i}\right) / k T\right) \quad p_{1}=n_{i} \exp \left(\left(E_{F i}-E_{t}\right) / k T\right)
$$

where $E_{t}$ and $E_{F i}$ are the trap energy level and the Fermi energy level for intrinsic materials, $N_{t}$ is trap density, $v_{p}$ is hole thermal velocity, $v_{n}$ is electron thermal velocity, $\sigma_{p}$ and $\sigma_{n}$ are crosssections for hole and electron capture by the trap. The SRH-recombination lifetime can be expressed as

$$
\tau_{S R H}=\frac{\tau_{p}\left(\Delta n+n_{0}+n_{i} e^{\left(E_{t}-E_{F i}\right) / k T}\right)+\tau_{n}\left(\Delta n+p_{0}+n_{i} e^{\left(E_{F i}-E_{t}\right) / k T}\right)}{p_{0}+n_{0}+\Delta n} .
$$

Next, radiative recombination takes place when an electron in the conduction band recombines with a hole in the valence band by emitting a photon. Since this process involves both electrons and holes, the recombination rate is proportional to the product to the electron and hole concentration. Radiative recombination dominates in direct band gap semiconductors. Thus, for indirect band gap semiconductor like $\mathrm{Si}$ has a very low radiative coefficient $\sim 1.1 \times$ $10^{-14} \mathrm{~cm}^{3} \mathrm{~s}^{-1}$ [186]. The rate of the radiative recombination, $R$, is given as

$$
R_{\text {rad }}=\beta\left(n p-n_{i}^{2}\right)
$$

where $\beta$ is the radiative coefficient. The radiative recombination lifetime, $\tau_{R A D}$ is given as

$$
\tau_{R A D}=\frac{1}{\beta\left(p_{o}+n_{o}+\Delta n\right)}
$$

Auger recombination is an intrinsically non-radiative process. In this recombination mechanism, an electron in the conduction band recombines with a hole in the valance band, the energy released during this process is transferred to a third carrier. The carrier particle is called 
Auger electron or Auger hole. The third carrier relaxes to its initial state by releasing its energy as phonons. Since this mechanism involves three carriers, this process becomes dominant at very high carrier injections. The Auger recombination rate can be expressed as

$$
R_{A U G}=C_{n} n\left(n p-n_{i}^{2}\right)+C_{p} p\left(n p-n_{i}^{2}\right)
$$

where $C_{n}$ and $C_{p}$ are the Auger Coefficients for electrons and holes respectively. The lifetime of this process is given as

$$
\tau_{A U G}=\frac{1}{\left(C_{p}+C_{n}\right) \Delta n^{2}}=\frac{1}{\gamma \Delta n^{2}} .
$$

Auger recombination via traps was discussed by Landsberg [187]. Here an electron in the conduction band makes a transition to the trap state and releases energy to a second electron.

The rate of recombination of excess electron-hole pairs can be expressed as follows

$$
\frac{d \Delta n}{d t}=-\frac{\Delta n}{\tau}
$$

where $\Delta n$ is the excess carrier concentration and $\tau$ is the recombination lifetime: the average time it takes for the system to revert to equilibrium. The net recombination rate consists of different recombination mechanisms and since they are additive the overall carrier lifetime can be written as follows

$$
\frac{1}{\tau}=\frac{1}{\tau_{S R H}}+\frac{1}{\tau_{R A D}}+\frac{1}{\tau_{\text {Auger }}}+\cdots
$$

\subsection{Pump-probe measurements of Chalcopyrite crystals}

The photoexcited carrier dynamics in the bulk CGP, ZGP, and CSP crystals were measured as a function of temperature and excitation fluence using TRTS. Details of the TRTS are discussed in section 2.8. Here the fractional change in terahertz transmission of the terahertz peak due to photoexcitation is recorded. Delay stage 1 is fixed at the peak of the THz pulse and pump delay stage 2 is varied. Then the difference between the transmitted electric field, $\Delta E(t)$, is measured as 
a function of pump-probe delay time. where $\Delta E(t)=E(t)-E_{0}(t), E(t)$ and $E_{0}(t)$ are the transmitted $\mathrm{THz}$ peak intensity through the sample with and without excitation respectively.
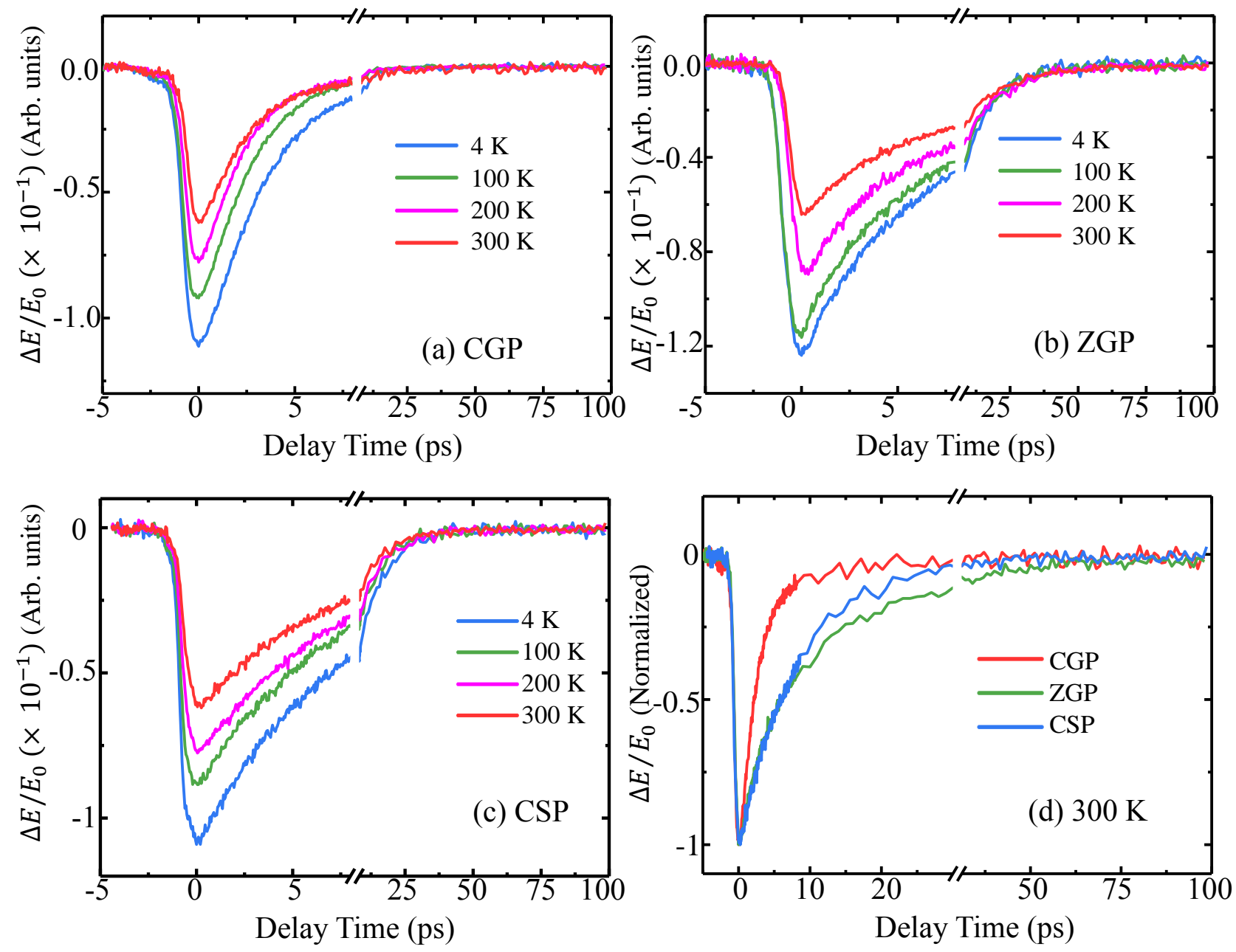

Figure 4.3. Differential transmission of the main peak of the THz probe as a function of pump-

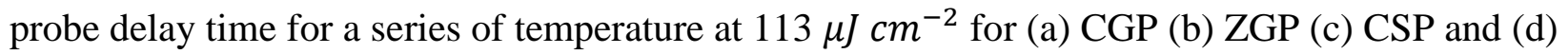
normalized differential transmission for all three crystals at $300 \mathrm{~K}$.

All three samples were photoexcited with a pump pulse at a wavelength of $400 \mathrm{~nm}$ and a spot size of $3 \mathrm{~mm}$. The terahertz radiation with spot size $1.5 \mathrm{~mm}$ is used as the probe beam. The pump spot size was chosen larger than the probe spot size to ensure a uniform excitation over the probed area. The samples were placed in the optical cryostat and their temperature was controlled between $4 \mathrm{~K}$ and $300 \mathrm{~K}$. The average pump fluence varying from $24 \mu \mathrm{Jm}^{-2}$ to $113 \mu \mathrm{J} \mathrm{cm}^{-2}$ using natural-density filters. 
Figure 4.3 shows the change in the transmitted $\mathrm{THz}$ peak electric field, $\Delta E / E_{0}$, as a function of pump-probe delay time for a series of temperatures ranging from $4 \mathrm{~K}$ to $300 \mathrm{~K}$ measured at $113 \mu \mathrm{Jm}^{-2}$ pump fluence for (a) CGP (b) ZGP and (c) CSP samples. In the absence of optical excitation, the conduction band has no carriers, hence THz probe cannot be absorbed. With the $400 \mathrm{~nm}(3.1 \mathrm{eV})$ optical pump, electrons can be excited from the valance band to the higher states of the conduction band of all three samples. Since there are many available states in the conduction band and valance band, these excited carriers absorb low energy THz probe. For all cases, $\Delta E / E_{0}$ increases quickly after photoexcitation then decay due to carrier recombination and trapping. It can be also observed that the $\Delta E / E_{0}$ decreases with increasing lattice temperature as sample absorption decreases with increasing temperature. Figure 4.3 (d) shows the normalized pump-probe transient spectra for all three crystals at $300 \mathrm{~K}$. Among the three samples CGP shows the fastest decay rate and ZGP shows the longest decay rate. This implies that the among three samples, CGP crystal has the highest defect concentration and the ZGP crystal has the lowest defect concentration.

Figure 4.4 (a-c) shows excitation fluence dependence of the $\Delta E / E_{0}$ of the THz probe for

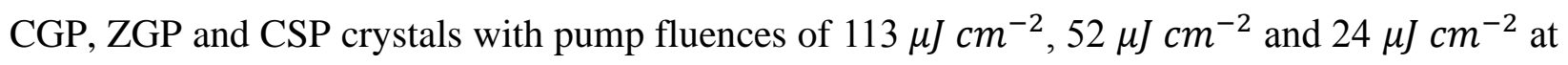
$300 \mathrm{~K}$. The observed increase in $\Delta E / E_{0}$ with increasing pump fluence is due to increases in excited carrier concentration. This will result in higher absorption at higher pump fluences. Figure 4.4 (df) shows normalized pump-probe transient spectra for CGP, ZGP, and CSP at different pump fluences. It can be observed that the carrier relaxation becomes faster as the pump fluence decrease.

Since relaxation dynamics exhibits an exponential decay, a double exponential function is used to fit the experimental transient as follows

$$
\frac{\Delta E}{E_{0}}=\sum_{i=1}^{2} \frac{A_{i}}{2} \exp \left(-\frac{t-t_{0}}{\tau_{i}}\right)
$$

where $A_{i}$ and $\tau_{i}$ are amplitude and decay time for the $i^{\text {th }}$ component. The parameters extracted from the bi-exponential fitting for CGP crystal are shown in figure 4.5 as a function of lattice temperature and pump fluence. Figure 4.5 shows (a) the extracted amplitude values for fast decay mechanisms. Amplitude values provide information about the fraction of carriers that participate in each decaying mechanism. The observed decrease in the magnitude of the fast decay amplitude, 
$\left(\left|A_{1}\right|\right)$, with increasing temperature is due to the decrease in sample absorption at the higher temperatures. It also indicates that the $\left|A_{1}\right|$ increases with increasing excitation fluence. Increasing pump-fluence results in higher excitation carrier density. Hence as the excitation fluence increases, the number of carriers participating in the relaxation process increases. As shown in figure 4.5 (b), slow decay amplitude, $\left(\left|A_{2}\right|\right)$, does not show any significant dependence on the temperature.
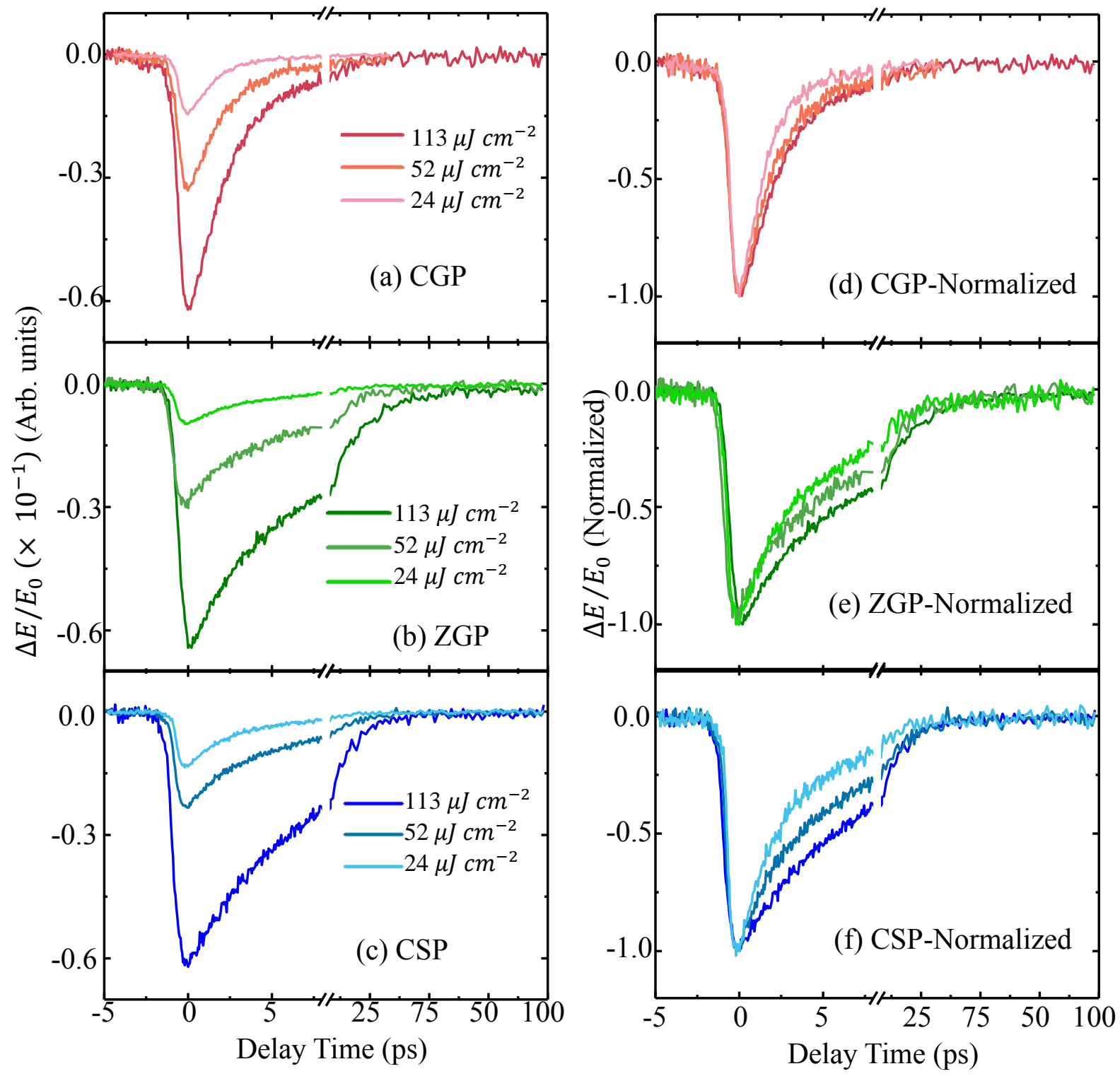

Figure 4.4. Differential THz transmission as a function of pump fluence for (a) CGP (b) ZGP and (c) CSP (d-f) shows normalized differential THz transient for CGP, ZGP and CSP respectively. 
Exponential fitting reveals two relaxation processes. The fast relaxation time, $\tau_{1}$, ranges from $2-4 \mathrm{ps}$ and slow relaxation time, $\tau_{2}$, ranges from $28-35 \mathrm{ps}$ for pump fluence $113 \mu \mathrm{J} \mathrm{cm}^{-2}$. $\tau_{1}$ is strongly depends on the carrier concentration and it decreases with temperature for the CGP sample. The thermally excited population is higher in the CGP sample since it a narrow bandgap material. Hence temperature affects the carrier relaxation in the CGP sample. The $\tau_{1}$ was attributed to carrier relaxation from higher excited states to the band edge. The slow decay time in all three samples is strongly dependent on the temperature and independent of the temperature. Since these samples have higher defect concentration, the slow decay time was attributed to SRH recombination. Figure 4.5 (c) shows the, $\tau_{1}$ and (d) shows the $\tau_{2}$ as a function of temperature and excitation fluence. With the pump fluence increasing, both $\tau_{1}$ and $\tau_{2}$ are increased. This is due to the saturation of available states and traps sites as the excitation fluence is increased $[188,189]$.
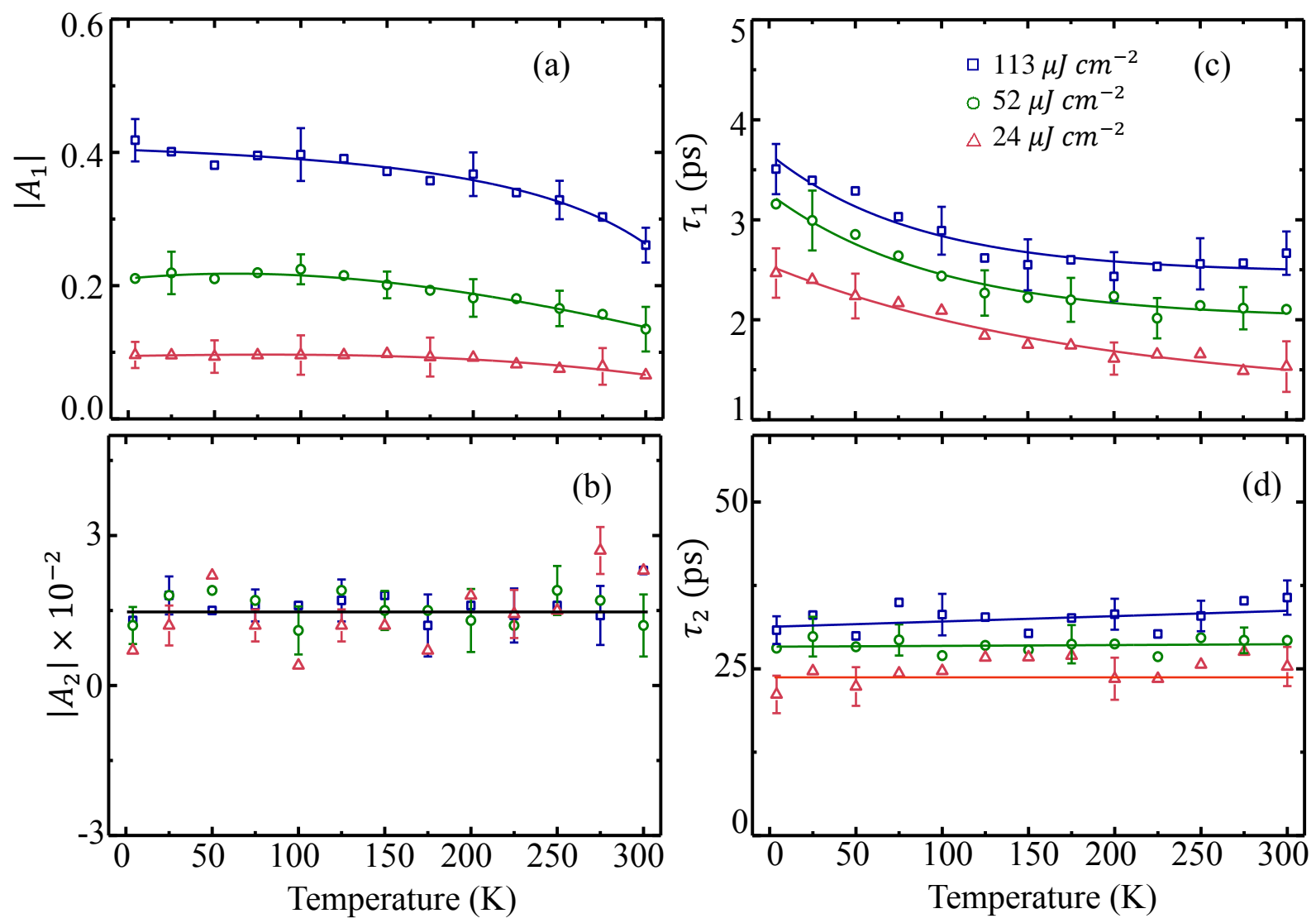

Figure 4.5. Temperature and excitation fluence dependence of extracted parameters from biexponential fitting for CGP crystal. (a) fast decay amplitude (b) slow decay amplitude (c) fast decay time (d) slow decay time 
Figure 4.6 (a) and (b) show extracted magnitude $\left|A_{1}\right|$ and $\left|A_{2}\right|$ as a function of temperature for ZGP crystal at several pump fluences. The magnitude of the $\left|A_{1}\right|$ decreases with increasing temperature while $A_{2}$ remains constant. Figure 4.6 (c) and (d) shows the $\tau_{1}$ and $\tau_{2}$ as a function of temperature for the ZGP sample. It can be seen that $\tau_{1}$ and $\tau_{2}$ increases with pump fluence, suggesting the saturation of states towards higher carrier density. The $\tau_{1}$ at $113 \mu \mathrm{J} \mathrm{cm}^{-2}$ and 52 $\mu J \mathrm{~cm}^{-2}$ pump fluences do not show any significant dependence over the temperature range $4 \mathrm{~K}$ to $300 \mathrm{~K}$. However, $\tau_{1}$ at $24 \mu \mathrm{J} \mathrm{cm}^{-2}$ pump fluence decrease with increasing temperature.
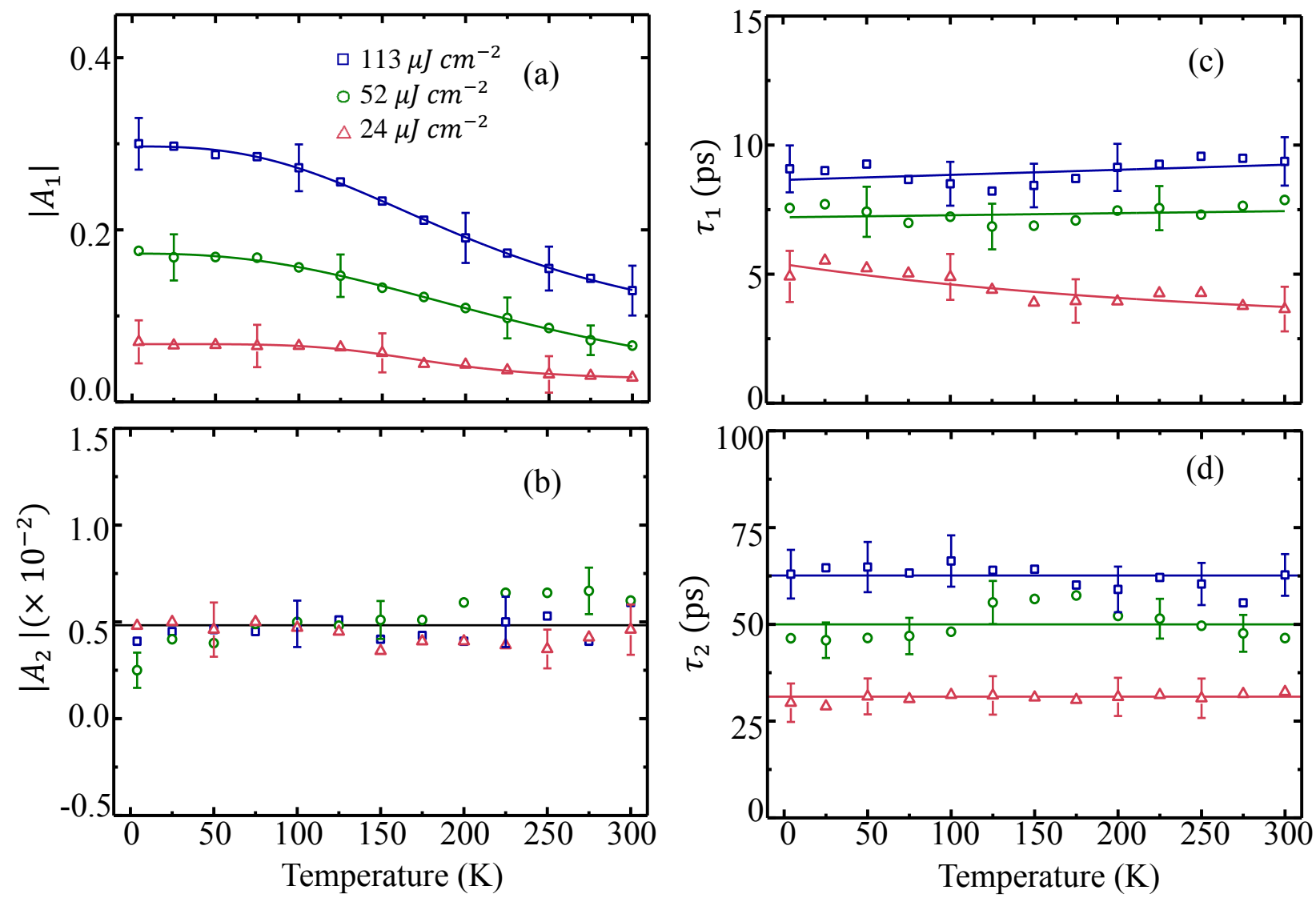

Figure 4.6. Temperature and excitation fluence dependence of extracted parameters from biexponential fitting for ZGP crystal. (a) fast decay amplitude (b) slow decay amplitude (c) fast decay time (d) slow decay time

Figure 4.7 shows the extracted parameters for the CSP sample as a function of temperature for three pump fluences. Figure 4.7 (a) and (b) shows extracted magnitude $\left|A_{1}\right|$ and $\left|A_{2}\right|$ over the 
temperature range $4 \mathrm{~K}$ to $300 \mathrm{~K}$. Temperature and pump fluence dependence of $\left|A_{1}\right|$ and $\left|A_{2}\right|$ for CSP sample are similar to ZGP and CGP samples. As shown in figure 4.7 (c) and (d), $\tau_{1}$ and $\tau_{2}$ does not show any significant dependence on the lattice temperature. It is seen that both $\tau_{1}$ and $\tau_{2}$ increases with increasing pump fluence. This effect results from the saturation of available states, as discussed above.
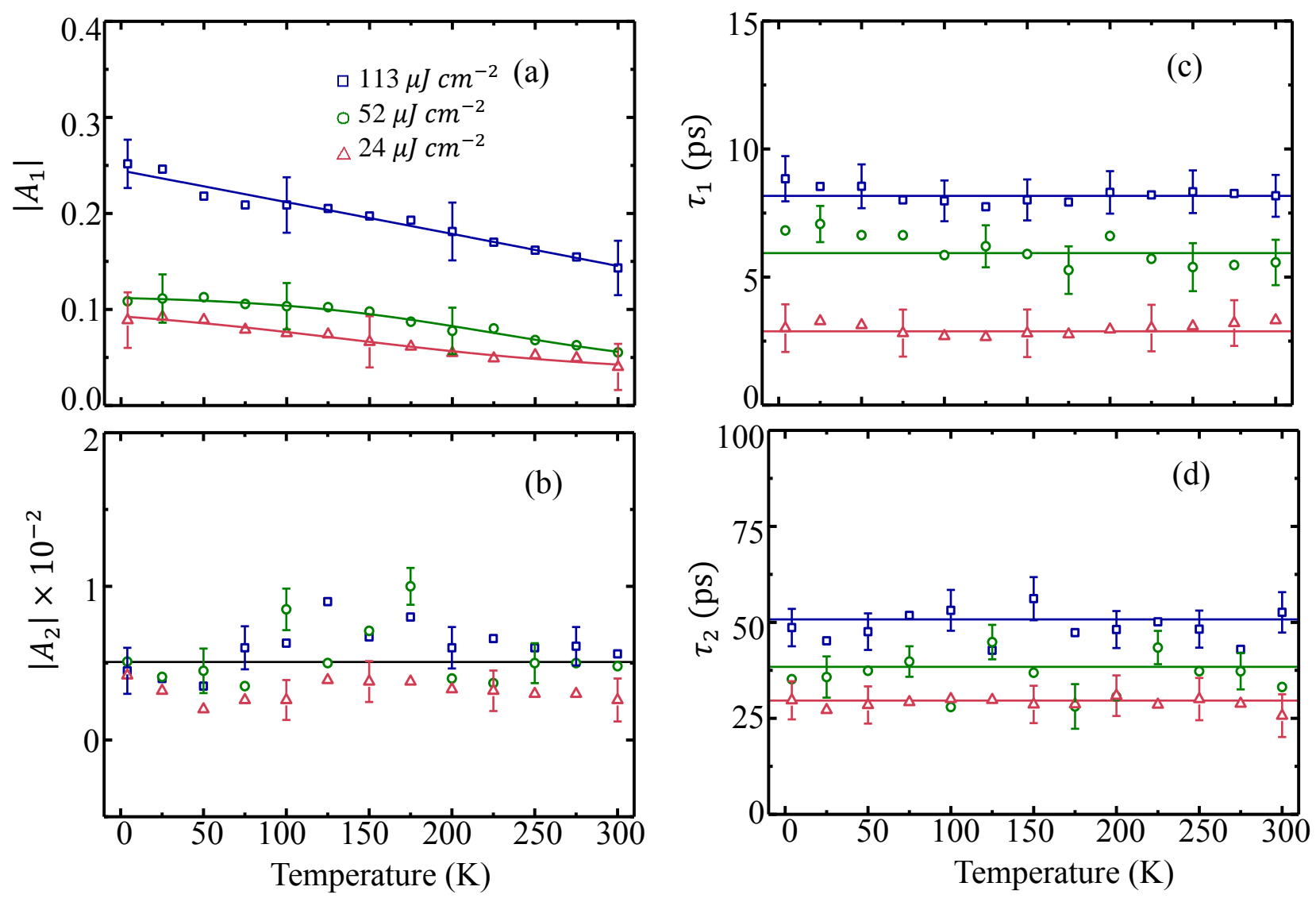

Figure 4.7 Temperature and excitation fluence dependence of extracted parameters from biexponential fitting for CSP crystal. (a) fast decay amplitude (b) slow decay amplitude (c) fast decay time (d) slow decay time

\subsection{Inversion Analysis Technique}

Generally, a transient is analyzed phenomenologically by fitting a multiexponential decay as discussed in the previous section. Multiexponential fittings are only valid in the low carrier density region and recombination mechanisms should be known in advance to select the suitable 
model. As discussed in section 4.2, the recombination processes like Auger, radiative, SRH recombination depend explicitly on the charge carrier density. Multiple exponential fittings cannot reveal the charge dynamics accurately, as the decay mechanisms of a semiconductor are nonexponential. The actual rate equation at higher carrier density can be written as follows

$$
\frac{d n}{d t}=\alpha_{1} n+\alpha_{2} n+\beta n^{2}
$$

where $\alpha_{1}$ is the decay coefficient for carrier relaxation to the band edge, $\alpha_{2}$ is the SRH coefficient, $\beta$ is the trap-assisted Auger coefficient. The rate equation 4.12 cannot fit with simple exponential functions.

J. Linnros proposed the inversion analysis technique to address these issues $[190,191]$. In this method, transient absorption data is transformed by taking the time derivative numerically. This inverts the measured transient-absorption data from the solution of the underlying rate equation back to the rate equation itself. By plotting the derivative of data as a function of excited carrier density, the underlying rate equation can be obtained. This allows one to obtained various recombination mechanisms in each carrier density regime. Furthermore, the instantaneous lifetime can be obtained by dividing the derivative by carrier density. Also, this allows obtaining the relaxation time constants without relying on the multi-exponential fitting.

$$
-\frac{d n}{d t}=n\left(\alpha_{1}+\alpha_{2}+\beta\right)=\frac{n}{\tau(n)} .
$$

To perform inversion analysis, the experimental absorption data must be smooth since noise is amplified when taking the derivative of the data. Then inversion analysis is performed by converting experimental transient absorption data into carrier density as follows

$$
n=-\frac{1}{\sigma d} \ln \left[1-a b s\left(\frac{\Delta E}{E}\right)\right]
$$

where $\sigma$ is the absorption cross-section, and $d$ is the sample thickness. Excited carrier density in the sample is calculated as follows

$$
n \approx \frac{I_{o} \cdot \alpha_{\text {pump }}}{\hbar \omega}
$$


where, $I_{0}$ is the incident flux in $\mathrm{J} / \mathrm{cm}^{2}, \hbar \omega$ is the pump energy and $\alpha_{\text {pump }}$ is the absorption coefficient in $\mathrm{cm}^{-1}$ at the pump energy. The absorption cross-section can be obtained by taking the slope from the graph of $I_{o} . \alpha_{\text {pump }} / \hbar \omega$ vs $1 / d(\Delta E / E)$ as shown in figure 4.8

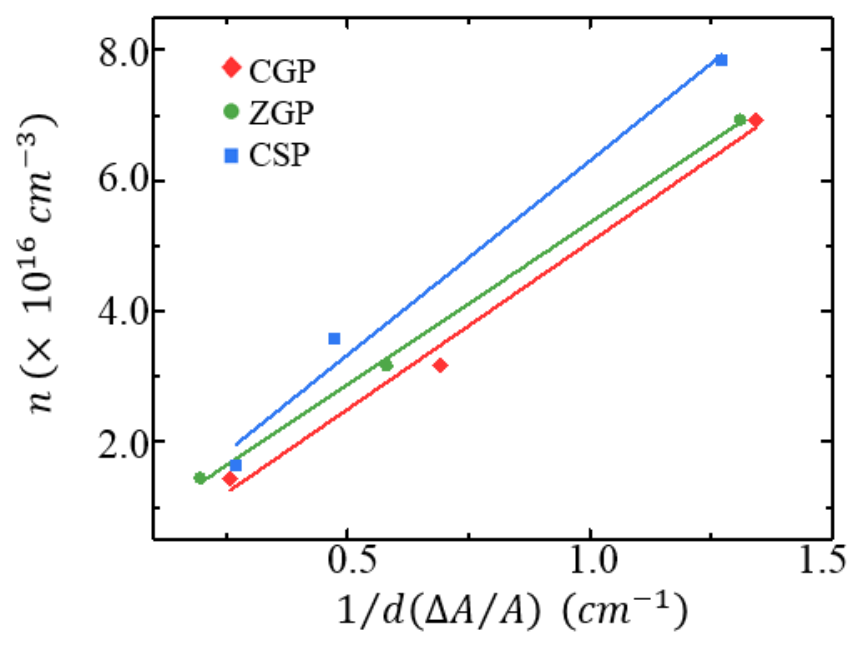

Figure 4.8. Graph of $n$ vs $1 / d(\Delta E / E)$ for three crystals

Figure 4.9 shows the inverted transient-absorption decay as a function of carrier density measured at several pump fluences for (a) CGP (b) ZGP and (c) CSP at $300 \mathrm{~K}$. The slope of the plot corresponds to the order of nonlinear relaxation mechanisms. After the photoexcitation, carriers relax from higher excited states to band edge via carrier-carrier scattering and carrierphonon interactions. This process is linearly proportional to the carrier density. As described in equation 4.12, SRH recombination also linearly proportional to the carrier density while the trapassisted auger recombination mechanism is proportional to the square of the carrier density. Hence, these recombination mechanisms will have slopes of 1 and 2 on a log-log plot. In figure 4.9, at high carrier density, the rate has a slope is 1, indicating carrier relaxation to the band edge. In medium carrier density, the rate has a slope of 2 . Possible second-order recombination mechanisms $\left(n^{2}\right.$ or $\left.p^{2}\right)$ are radiative recombination and trap-assisted Auger recombination. The radiative recombination rate is proportional $n p$.Trap-assisted auger recombination rate proportional to $n N_{T}$. Photoluminescence experimental was carried out to study radiative emission from three crystals. However, photoluminescence was not observed in these three samples. Due to a lack of radiative 
emission from the experiment, radiative recombination was ruled out. We attributed second-order processes to trap-assist Auger recombination. All three crystals have excess photon energies after the photoexcitation hence it is possible to take place these fast recombination processes. In the lower carrier densities, the slope reduces to 1 , indicating the recombination is due to SRH. The position of the slope shows an upward shift with decreasing pump fluence. This reveals that the carrier relaxation becomes faster with decree sing pump fluence.
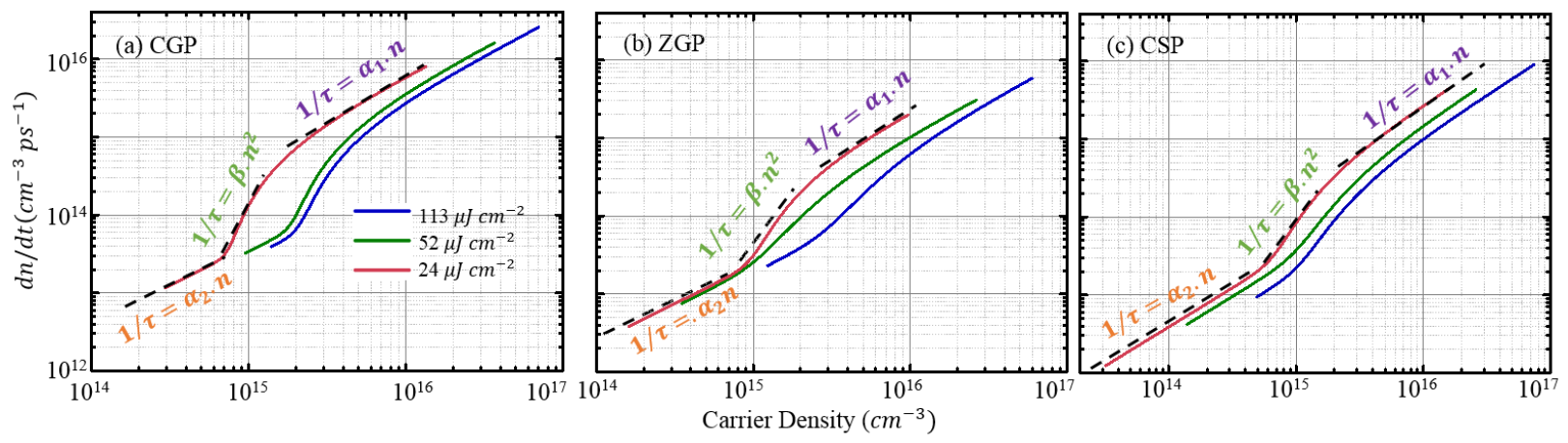

Figure 4.9. The inverted spectra showing the relaxation kinetics of (a) CGP (b) ZGP and (c) CSP as a function of carrier density for several pump fluences.

Table 01. Rate constants for three crystals

\begin{tabular}{cccc}
\hline Sample & $\alpha_{1}\left(p s^{-1}\right)$ & $\alpha_{2}\left(p s^{-1}\right)$ & $\beta\left(\mathrm{cm}^{-3} p s^{-1}\right)$ \\
\hline CGP & $3.68 \times 10^{-1}$ & $3.50 \times 10^{-2}$ & $4.44 \times 10^{-17}$ \\
ZGP & $1.06 \times 10^{-1}$ & $2.14 \times 10^{-2}$ & $1.40 \times 10^{-17}$ \\
CSP & $1.24 \times 10^{-1}$ & $2.10 \times 10^{-2}$ & $2.19 \times 10^{-17}$ \\
\hline
\end{tabular}

Figure 4.10 shows instantaneous lifetime $\tau(n)$ as a function of carrier density for several pump fluences. It can be observed that for all cases the lifetime is shorter at higher carrier density and it flattens off at low carrier densities. It can be noted that carrier lifetime increases with increasing pump fluence for all three crystals. The instantaneous lifetime results also confirmed that the CGP sample exhibits decay rates for all recombination mechanisms that are faster than that of the ZGP and CSP samples. 

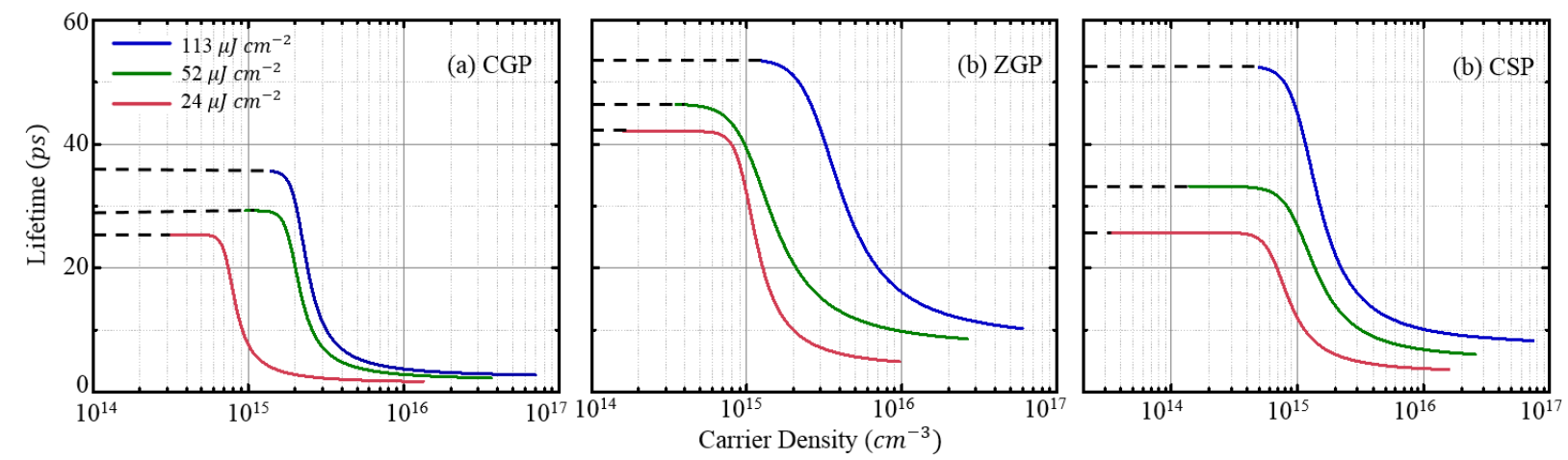

Figure 4.10. Instantaneous lifetimes as a function of carrier density for (a) CGP (b) ZGP and (c) CSP as a function of carrier density for several pump fluences.

\subsection{Photoconductivity Measurements}
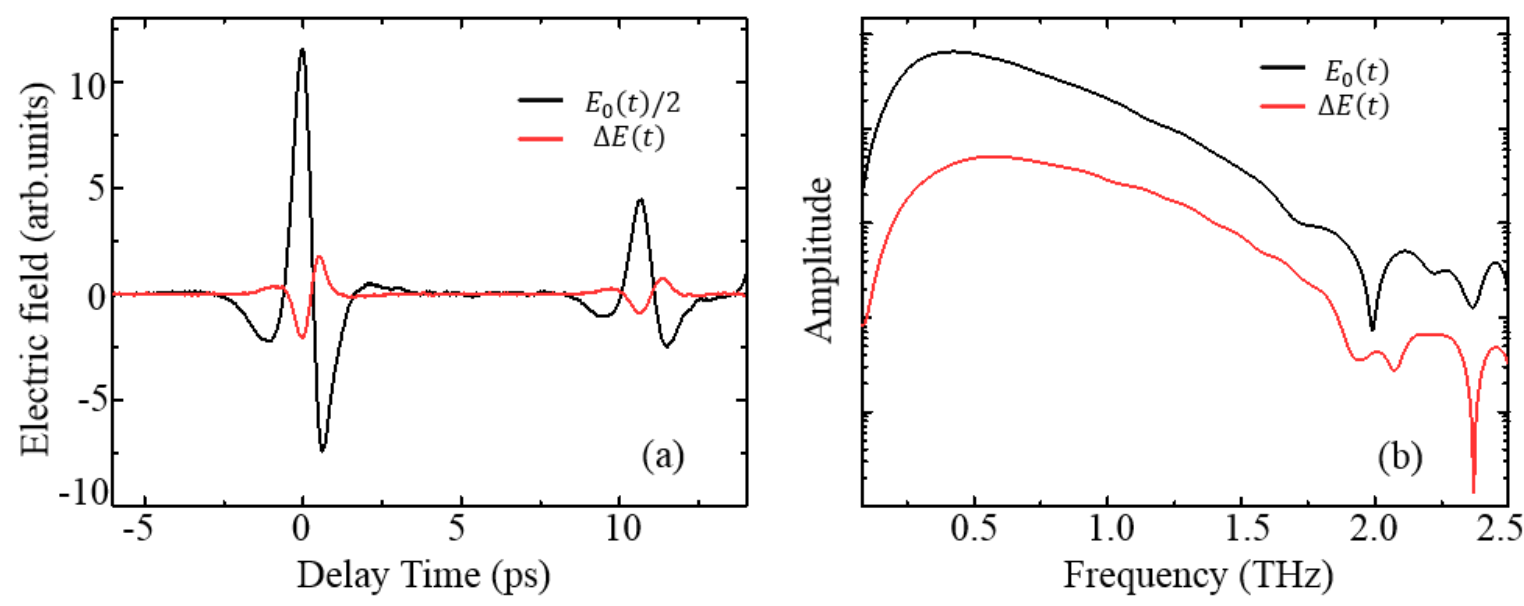

Figure 4.11. The black graph shows the $\mathrm{THz}$ electric field transmitted through CSP sample without excitation. The red graph shows the differential signal $\Delta E(t)$. (b) shows corresponding amplitude spectra

In the previous section, the fractional change in the peak of the $\mathrm{THz}$ pulse is measured. In this section, the entire photoconductivity spectra at a fixed time after the photoexcitation are discussed. Details of the experimental setup are discussed in section 2.8. To measure the frequency response, pump-probe delay stage (D2) is fixed, and record the fractional change in terahertz transmission, $\Delta E(t) / E_{0}(t)$, by varying the delay time between the THz gate and trigger (D1). 
Here, all three samples were excited with a $400 \mathrm{~nm}$ pulse. The spot size of the pump pulse was 3 $\mathrm{mm}$ and the spot size of the terahertz beam was $1.5 \mathrm{~mm}$. Photoinduced conductivity of all three crystals was measured as a function of lattice temperature and pump-probe delay time.
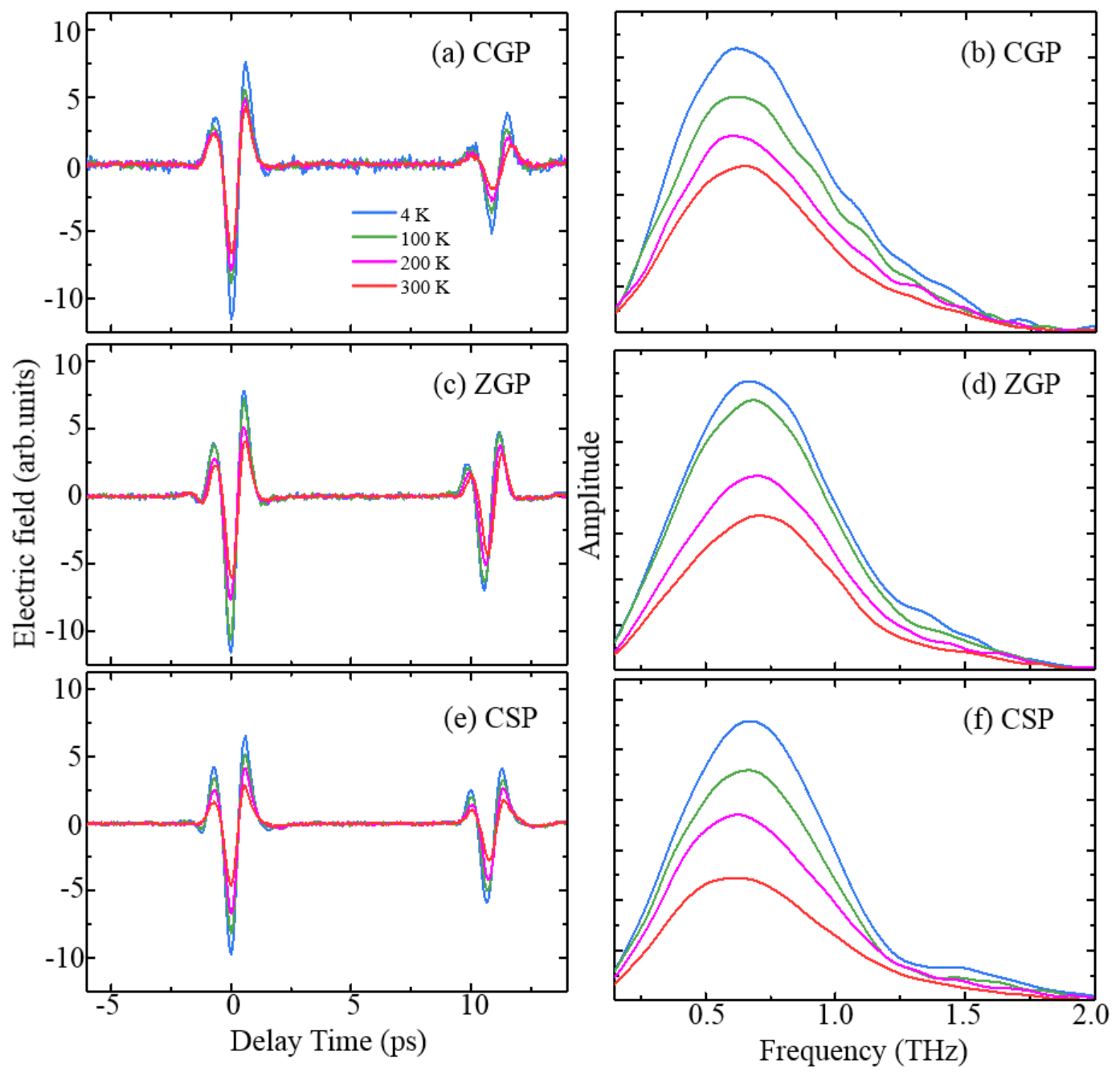

Figure 4.12 . Pump-induce changes in Terahertz electric filed for (a) CGP (c) ZGP (e) CSP as a function of temperature. (b) shows corresponding amplitude spectra for (b) CGP (d) ZGP and (f) CGP samples

Figure 4.11 (a) shows an example of $\mathrm{THz}$ electric field through the CSP sample in the absence of photoexcitation, $E_{0}(t)$, (black) and pump induced changed after excitation, $\Delta E(t)$, (red). The $E_{0}(t)$ has been scaled for visual aid. Figure 4.11 (b) shows the corresponding field amplitude spectra from the numerical FFT. With $400 \mathrm{~nm}$ excitation, the amplitude of the 
differential signal is less than $6 \%$ of the peak of the transmitted $\mathrm{THz}$ electric field without optical excitation. This is due to the increase in free carrier concentration by the photoexcitation. This will lead to increased free carrier absorption and decreased $\mathrm{THz}$ transmission.

Figure $4.12(\mathrm{a}, \mathrm{c}, \mathrm{e})$ shows $\Delta E(t)$ for all three crystals at $0 \mathrm{ps}$ after optical excitation for several temperatures. Figure $4.12(\mathrm{~b}, \mathrm{~d}, \mathrm{f})$ shows the FFT of the amplitude spectrum of $\Delta E(t)$. The $\Delta E(t)$ decreases with increasing temperature due to the decrease in sample absorption.
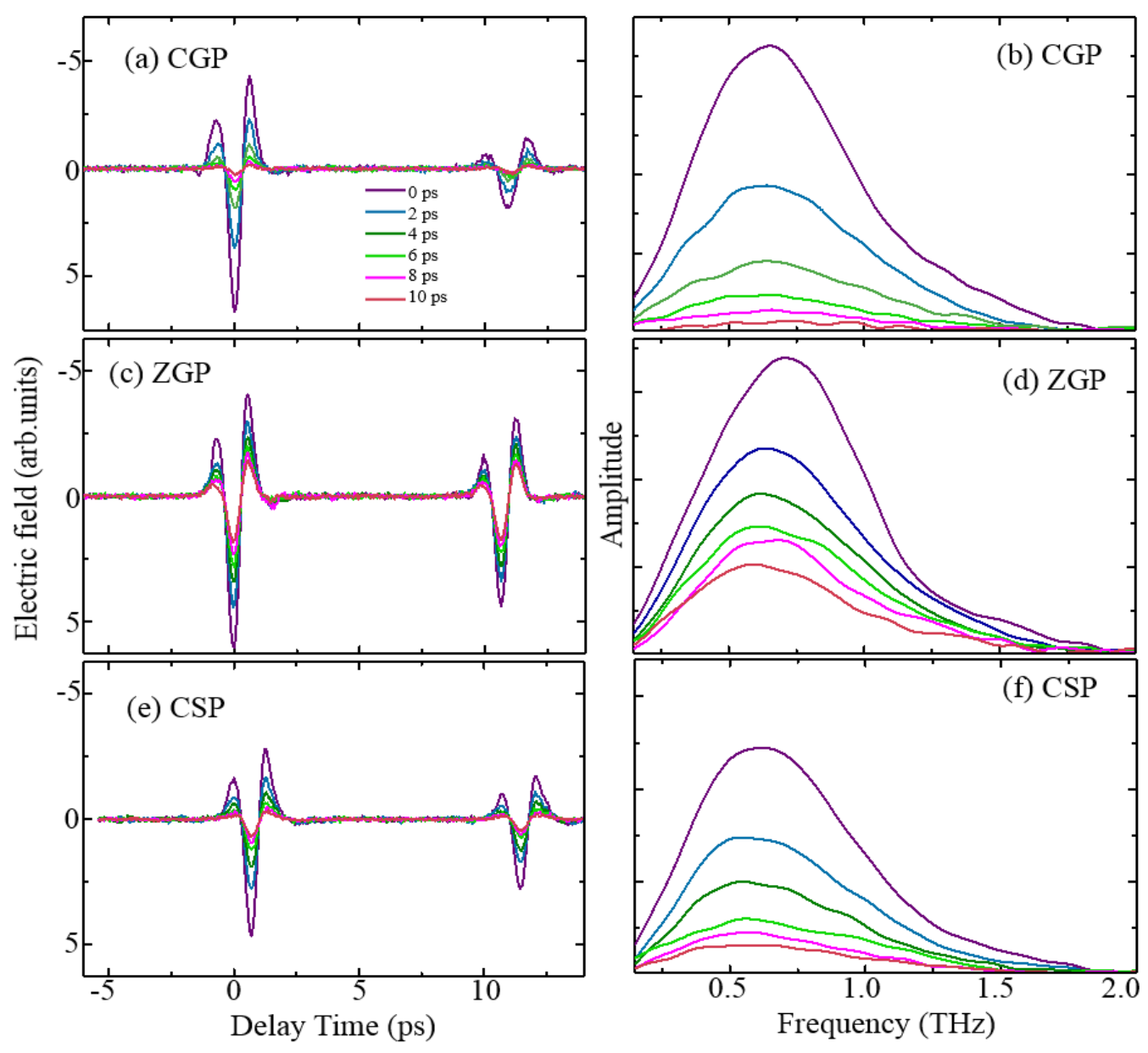

Figure 4.13. The differential signal $\Delta E(t)$ for (a) CGP (c) ZGP (e) CSP samples at various pumpprobe delay times. Amplitude spectra in the frequency domain after applying the FFT for (b) CGP (d) ZGP and (f) CSP 
Figure 4.13 (a,c,e) shows $\Delta E(t)$ for three crystals at 0 ps, 2 ps, 4 ps, 6 ps, 8 ps, and 10 ps after excitation. Figure $4.13(\mathrm{~b}, \mathrm{~d}, \mathrm{f})$ shows the FFT of the amplitude spectrum of $\Delta E(t)$. It is apparent from figure 4.13 that measured $\Delta E(t)$ decreases with increasing pump delay time for all three crystals. This is due to the recombination and trapping of carriers. For increasing time delays, carriers are already in their recombination phase. As a result, the free carrier concentration in the conduction band decreases with increasing pump delay time.

The frequency-dependent photoconductivity, $\Delta \tilde{\sigma}(\omega)=\sigma_{1}(\omega)+i \sigma_{2}(\omega)$ can be extracted from the temporal profile of the $\mathrm{THz}$ electric field with and without optical excitation as follows

$$
\frac{\Delta E(\omega)}{E_{0}(\omega)}=\frac{n_{s}+1}{n_{s}+1+Z_{0} \Delta \tilde{\sigma}(\omega) d},
$$

where $\Delta E(\omega)$ is Fourier transform of the differential signal, $E_{0}(\omega)$ is the Fourier transform of the terahertz electric field without photoexcitation, $n_{s}$ is the refractive index of the sample without photoexcitation, $Z_{0}$ is the impedance of the free space and $d$ is the thickness of the photoexcited region.
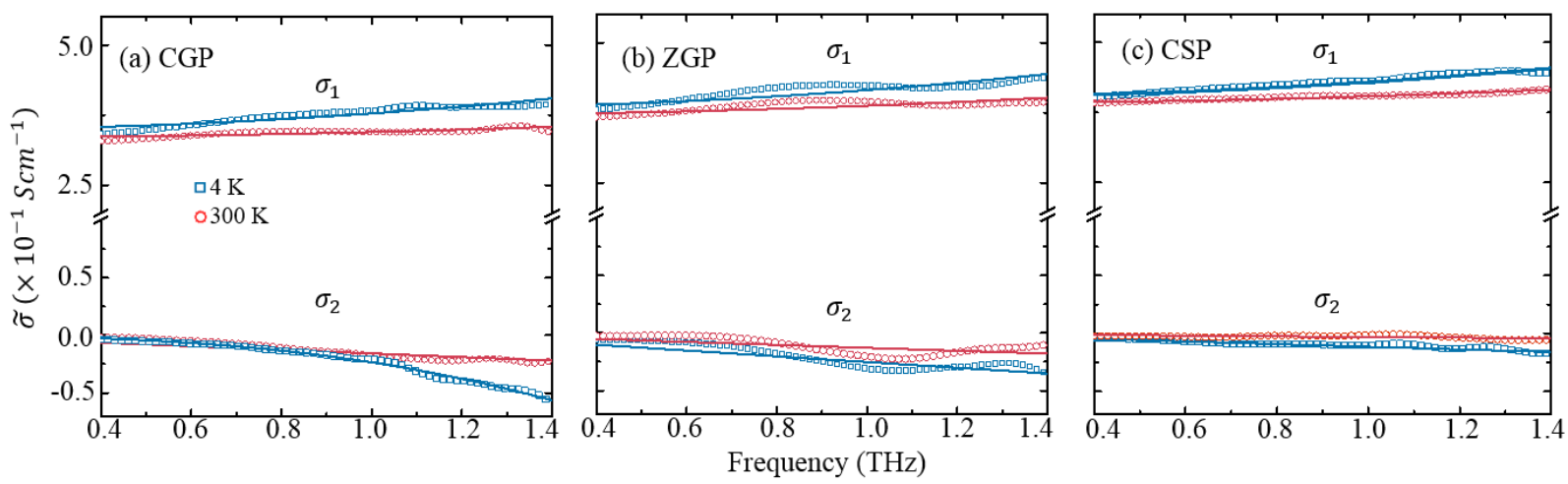

Figure 4.14. Real $\left(\sigma_{1}\right)$ and imaginary parts $\left(\sigma_{2}\right)$ of AC photoconductivity for (a) CGP (b) ZGP and (c) CSP crystals with lattice temperatures of $4 \mathrm{~K}$ and $300 \mathrm{~K}$ at 0 ps after excitation, fit by Drude-Smith conduction model.

The frequency-dependent complex photoconductivity for three crystals is extracted using equation 4.19. Figure 4.14 shows the real and imaginary parts of AC photoconductivity measured at 0 ps after excitation. It can be observed that there is an increase in the complex conductivity at 
$4 \mathrm{~K}$ compared to $300 \mathrm{~K}$. At higher lattice temperatures, photoexcited conductive electrons experience increased scattering from the lattice phonons which results in decreased conductivity.

Figure 4.15 shows the AC photoconductivity spectra for three crystals measured at $300 \mathrm{~K}$ for several pump-probe delay times. The real and imaginary parts of photoconductivity decay with increasing pump-probe delay time for all three crystals. As the delay time increases, the free-carrier concentration decreases due to carrier trapping and recombination. The photoinduced changes in conductivity are directly related to the carrier density hence AC photoconductivity decrease with increasing pump-probe delay time.
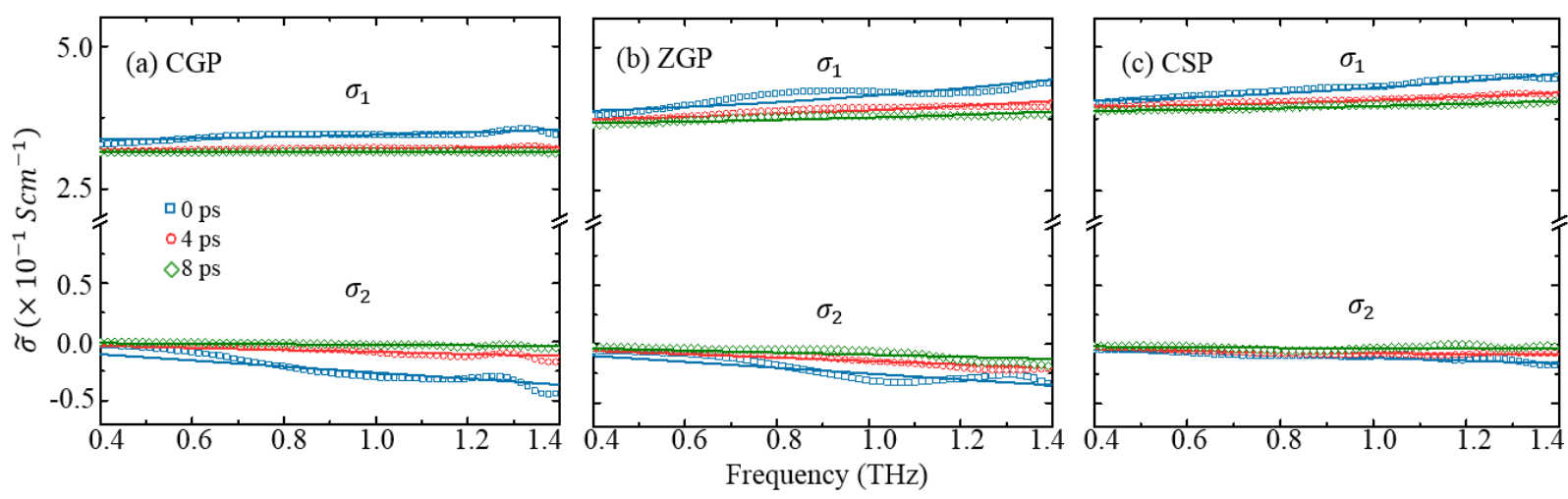

Figure 4.15. Real $\left(\sigma_{1}\right)$ and imaginary parts $\left(\sigma_{2}\right)$ of AC photoconductivity for (a) CGP (b) ZGP and (c) CSP crystals taken at several pump-probe delay times at $300 \mathrm{~K}$, fit by a Drude-Smith conduction model

For all three crystals $\sigma_{1}(\omega)$ is positive and increasing with frequency, whereas $\sigma_{2}(\omega)$ is negative and decreasing with frequency. The observed negative $\sigma_{2}(\omega)$ is due to scattering of the free charge carriers from the crystallite surfaces or grain boundaries, defects, dislocation, and vacancies. The shape of the photoconductivity spectra is the same as that for ground-state conductivity spectra. Experimentally obtained $\sigma_{1}(\omega)$ and $\sigma_{2}(\omega)$ spectra strongly deviate from the Drude model. Since $\sigma_{2}(\omega)$ is negative, photoconductivity response is described using DrudeSmith model. The experimental AC photoconduction results are well fit by the Drude-Smith model. Carrier density, $N$, scattering time, $\tau$, and backscattering coefficients, $c$, are extracted from the fitting. 
Figure 4.16 shows the $N$ as a function of delay time at $4 \mathrm{~K}$ and $300 \mathrm{~K}$ temperatures. The data points in figure 4.16 show the extracted $N$ from the Drude-Smith model. The differential THz transmission is directly related to the carrier density. The corresponding differential $\mathrm{THz}$ transmission signal is converted into carrier density using equations 4.14 and shown as a solid thick line in figure 4.16. The extracted carrier density follows the same trend as the differential $\mathrm{THz}$ transmission. Carrier density has its maximum value at 0 ps and then decays as carriers trapped or recombine. There is a decrease in carrier density with increasing temperature due to the absorption of the $\mathrm{THz}$ probe by thermally generated carriers.
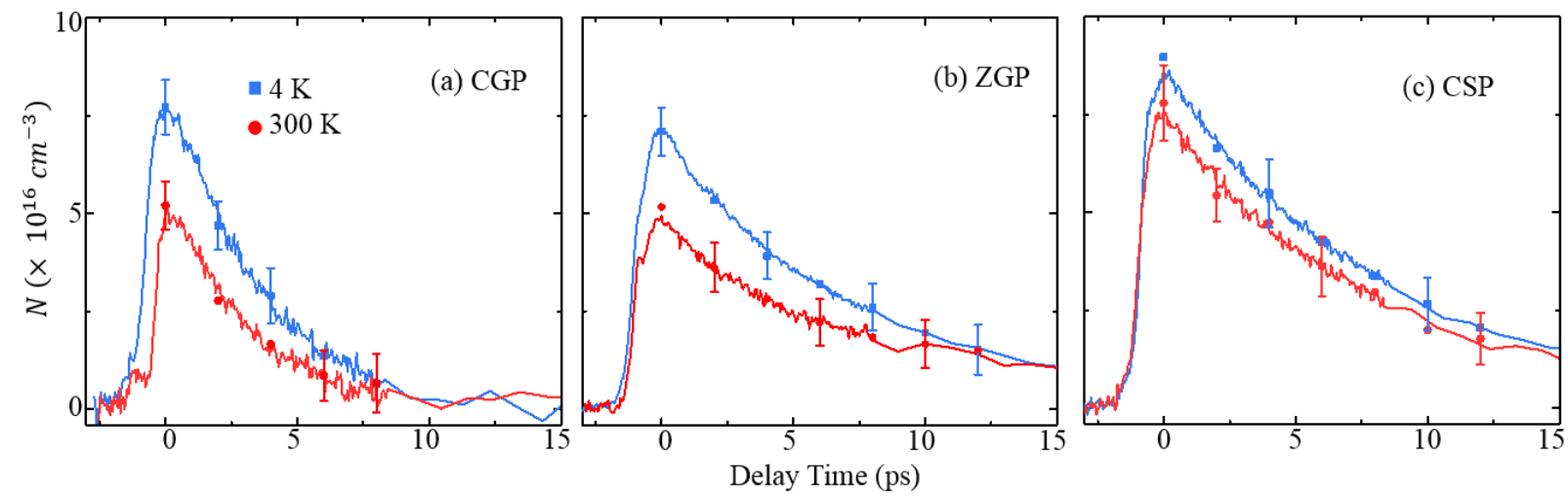

Figure 4.16. The carrier density as a function of pump-probe delay time at $4 \mathrm{~K}$ and $300 \mathrm{~K}$ for (a) CGP (b) ZGP and (c) CSP

\subsubsection{Carrier Relaxation and Mobility}
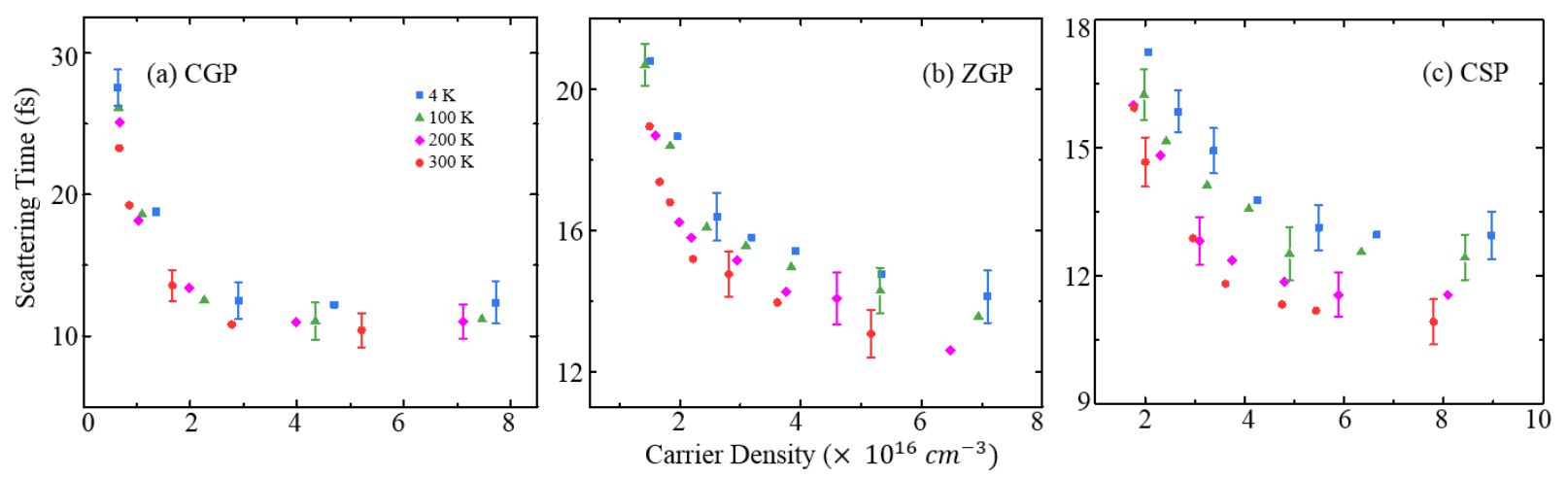

Figure 4.17. Carrier-density dependent scattering time $\tau$ at several temperatures for (a) CGP (b) ZGP (c) CSP 
Carrier mobility and relaxation times are crucial parameters in semiconductor devices. They play important role in determining the efficiency of devices. As described in section 3.9, carrier relaxation includes various scattering processes such as carrier-carrier scattering, scattering due to defects, and electron-phonon scattering. Carrier-carrier scattering is the dominant scattering mechanism in a non-equilibrium condition. Figure 4.17 shows the extracted scattering time as a function of carrier density at several temperatures. Scattering time decreases with increasing carrier density and temperature for all three crystals.
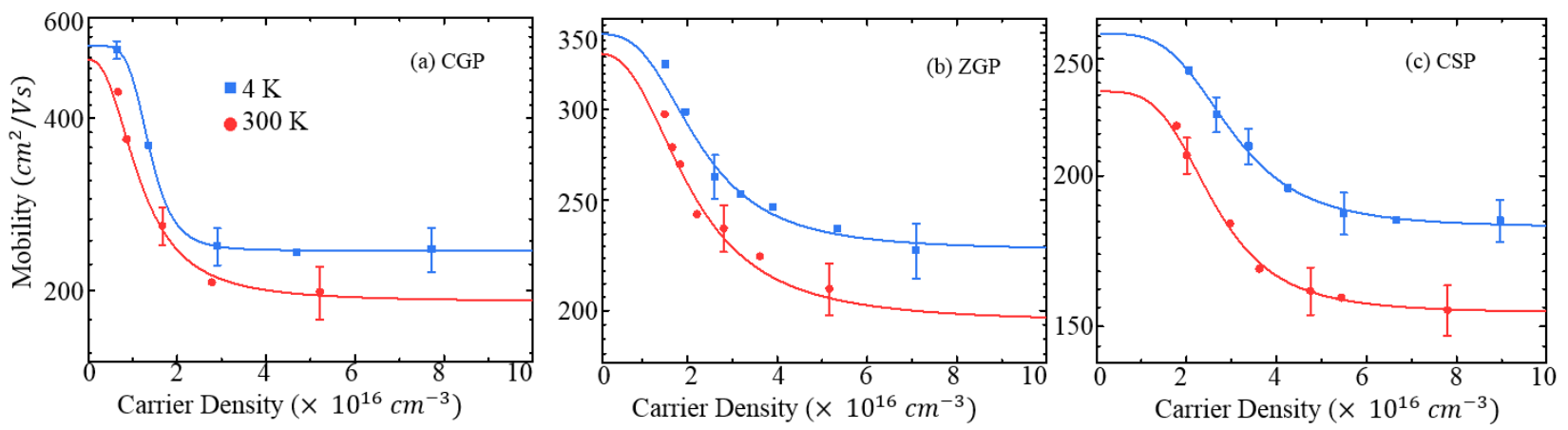

Figure 4.18. Carrier-density dependent mobility fit with a Caughey-Thomas relation at several temperatures for (a) CGP (b) ZGP and (c) CSP

In figure 4.18 shows the mobility, $\mu=e \tau / m^{*}$, determined from the scattering time as a function of carrier density for a series of temperatures for all three crystals. Carrier dependence of the mobility can be fit well to the empirical Caughey-Thomas [192].relation as follows

$$
\mu=\frac{\mu_{\max }-\mu_{\min }}{1+\left(\frac{N}{N_{\text {ref }}}\right)^{\alpha}}+\mu_{\min }
$$

where $\mu_{\max }$ and $\mu_{\min }$ are low and high carrier density mobility, $N_{\text {ref }}$ is the inflection density and $\alpha$ is the slope of the fit. At higher lattice temperatures carrier relaxation is faster as there is increased scattering from phonons and thermally excited carriers. This will result in lower mobility at higher temperatures. Mobility decreases with increasing carrier density. This is because carriers experience more scattering at higher carrier concentrations. At lower carrier densities, mobility 
levels off $\left(\mu_{\max }\right)$ as carriers behave independently of each other. The saturation of mobility at high carrier densities $\left(\mu_{\text {min }}\right)$ is due to the screening effect of Coulomb interactions Table 1 and Table 2 shows the extracted $N_{\text {ref }}$ and $\alpha$ values from the fitting for three crystals.

Table 02: Extracted $N_{\text {ref }}$ from mobility fitting for three crystals

\begin{tabular}{cccc}
\hline Temperature $(\mathrm{K})$ & $\mathrm{CGP}\left(\times 10^{15} \mathrm{~cm}^{-3}\right)$ & $\mathrm{ZGP}\left(\times 10^{16} \mathrm{~cm}^{-3}\right)$ & $\mathrm{CSP}\left(\times 10^{16} \mathrm{~cm}^{-3}\right)$ \\
\hline 4 & 3.27 & 3.77 & 2.85 \\
100 & 2.64 & 1.37 & 2.34 \\
200 & 3.03 & 4.46 & 2.51 \\
300 & 3.08 & 1.59 & 2.05 \\
\hline
\end{tabular}

Table 03: Extracted $\alpha$ from mobility fitting for three crystals

\begin{tabular}{cccc}
\hline Temperature $(\mathrm{K})$ & CGP & ZGP & CSP \\
\hline 4 & 2.18 & 1.70 & 2.76 \\
100 & 2.73 & 1.53 & 2.83 \\
200 & 1.73 & 1.45 & 2.86 \\
300 & 1.66 & 1.84 & 2.99 \\
\hline
\end{tabular}

Figure 4.19 shows the extracted $c$ for three samples at $4 \mathrm{~K}$ and $300 \mathrm{~K}$ as a function of carrier density. For all cases, $c$ increases with increasing carrier density, which indicates strong carrier backscattering associated with carrier occupancy. As carrier density increases, carriers experience more backscattering. Carrier density-dependent back-scattering coefficient shows similar behavior as mobility. Hence, the Caughey-Thomas relation is also used to fit the carrier density-dependent backscattering coefficients.

$$
c=\frac{c_{\max }-c_{\min }}{1+\left(\frac{N}{N_{\text {ref }}}\right)^{\alpha}}+c_{\min }
$$

where, $c_{\max }$ and $c_{\min }$ are low and high carrier density backscattering coefficients. Our measurements show that the backscattering coefficient strongly dependent on the carrier density. Similar behavior is observed by Wang et. al [193]. However, we could not well justify the carrier density dependence of the backscattering coefficient. To discuss the observed AC 
photoconductivity other conductivity models like the Boltzmann conductivity model [193] should be used.
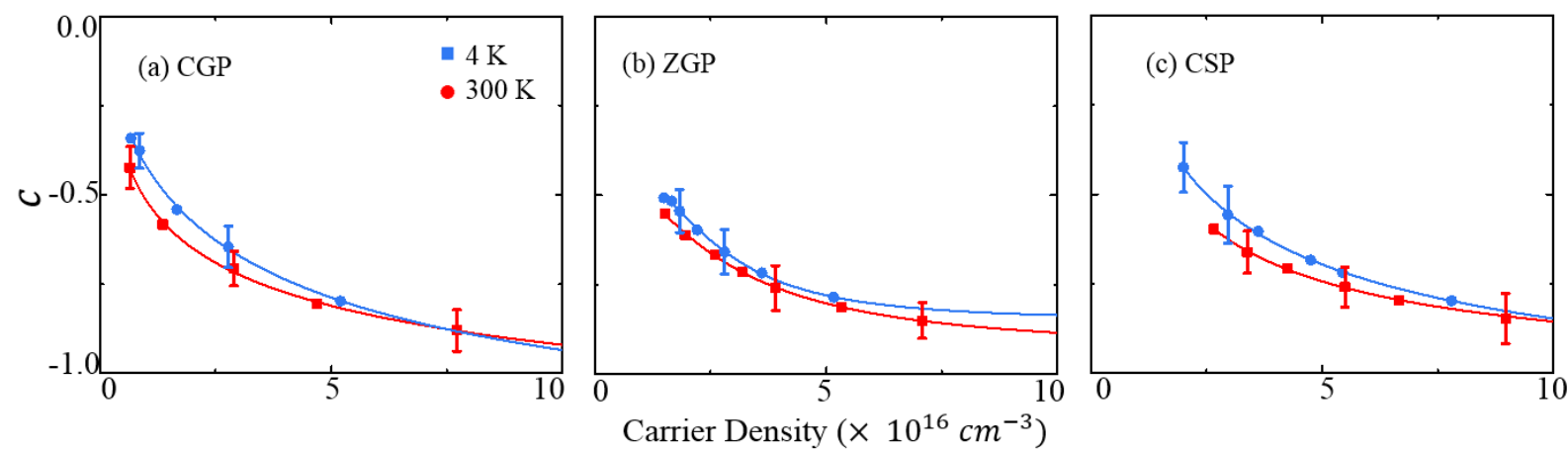

Figure 4.19. Carrier density-dependent backscattering coefficient for all three crystals measured at $4 \mathrm{~K}$ and $300 \mathrm{~K}$

Table 04: Extracted $N_{r e f}$ from backscattering coefficient fitting for three crystals

\begin{tabular}{cccc}
\hline Temperature $(\mathrm{K})$ & $\mathrm{CGP}\left(\times 10^{15} \mathrm{~cm}^{-3}\right)$ & $\mathrm{ZGP}\left(\times 10^{15} \mathrm{~cm}^{-3}\right)$ & $\mathrm{CSP}\left(\times 10^{15} \mathrm{~cm}^{-3}\right)$ \\
\hline 4 & 2.23 & 2.46 & 2.44 \\
100 & 2.40 & 2.45 & 2.28 \\
200 & 2.07 & 2.74 & 3.70 \\
300 & 2.62 & 2.51 & 3.63 \\
\hline
\end{tabular}

Table 05: Extracted $\alpha$ backscattering coefficient fitting for three crystals

\begin{tabular}{cccc}
\hline Temperature $(\mathrm{K})$ & CGP & ZGP & CSP \\
\hline 4 & 2.29 & 1.61 & 1.69 \\
100 & 1.93 & 1.83 & 2.83 \\
200 & 1.74 & 3.05 & 1.90 \\
300 & 2.64 & 2.42 & 2.15 \\
\hline
\end{tabular}

\subsection{Chapter Summary}

In this chapter, ultrafast carrier dynamics and frequency-dependent photoexcited conductivity response of CGP, ZGP, and CSP chalcopyrite crystals have been investigated using TRTS. The multiexponential fitting reveals two relaxation processes that contribute to the decay of photoexcited carriers. It is observed that the lifetime strongly depends on the carrier density. 
Saturation of available states at higher pump fluences leading to a longer lifetime. By applying inversion analysis on the experimental transient, carrier density-dependent recombination mechanisms were obtained

The AC photoconductivity is measured as the function of pump-probe delay time and lattice temperature. The decrease of photoconductivity with increasing temperature is due to an increase in phonon density. The AC photoconductivity of all samples is well fitted by the DrudeSmith model. Carrier density decreases with pump-probe delay time since photoexcited carriers recombine or trapped. At higher temperatures, carriers experience more scattering, resulting in lower mobilities. Carrier density-dependent mobility and backscattering coefficients are well fitted with the Caughey-Thomas relation. This detailed understanding of carrier dynamics and photoconductivity is crucial to potential applications of CGP, ZGP, and CSP chalcopyrite crystal. 


\section{Chapter 5: Conclusion}

\subsection{Summary of Thesis}

In this thesis, ground state and excited state charge-carrier dynamics and transport mechanisms in CGP, ZGP and CSP chalcopyrite crystals have been investigated using Terahertz spectroscopy. II-IV-V $V_{2}$ chalcopyrite crystals are one material class that has been interesting to the

optical community. An understanding of optical, electronic properties, and carrier dynamics is essential to achieve high performance of semiconductor devices such as photovoltaic devices, spintronic devices, photodiodes, etc.

Temperature-dependent THz-TDS measurements in CGP, ZGP, and CSP samples provide $\mathrm{THz}$ refraction, absorption, electron-phonon coupling, AC conductivity, carrier mobility, and scattering mechanisms that are consistent with direct gap semiconductors. Our measurements indicate that the optical and electronic properties are significantly influenced by the temperature that affects the underlying carrier scattering mechanisms. Refractive index increases with the increasing temperature due to the modification of the refractive index by electrons and phonons at different temperatures. Electron-lattice interactions are modeled by Bose-Einstein statics which gives average phonon frequency and the electron-phonon coupling constants. $\mathrm{THz}$ absorption reveals the center frequencies of the lowest-lying optical phonon modes for each temperature. Understanding these properties could lead to the design and fabrication of devices with improved performance.

Temperature-dependent AC conductivity measurements allow a deeper understanding of microscopic transport without contacts. AC conductivity provides ground-state carrier density and scattering time. Electron concentration arising from the occupancy that follows Fermi Dirac distribution. It provides information about dopant concentration and their binding energies. Halleffect measurement analysis of the mobility (or scattering times) allows for mapping of the temperature-dependent origins of the carrier-scattering mechanisms whether that be from atoms, electrons, phonons, or dislocations. This approach overcomes poor experimental reliability of contact-based Hall-effect measurements of chalcopyrite crystals due to their low mobility. Moreover, this study defines various parameters for many scattering mechanisms that have not 
been previously reported. This study also supports detailed measurements of defects in these chalcopyrite semiconductors, offering material characterization to improve bulk crystal growth for photonic and nonlinear optical applications. Also, this method can be used to investigate materials that are difficult to study with conventional methods.

Optical pump-THz probe measurements provide information on the recombination mechanisms in these crystals. Analysis of the differential THz transient shows a two-component exponential relaxation, with decay times that increase with pump fluence due to state filling. Among three crystals, the CGP sample shows the fastest decay indicating it has the highest defect concentration. Inverting the transients recaptures the canonical rate-equation for the system and from which the recombination dynamics are attributed to the entire excitation range.

TRTS measurements show that AC photoconductivity decreases with increasing temperature due to an increase in phonon interactions. AC photoconductivity provides excitedstate carrier density, scattering time, and backscattering coefficient. Our measurements show that the carrier mobility in samples is considerably influenced by temperature and carrier density. This detailed understanding of carrier dynamics and photoconductivity is crucial to potential applications of chalcopyrite crystals. This work has shown that TRTS can be used to measure the carrier dynamics and mobility in a photoexcited sample.

\subsection{Future Research}

While most of the carrier and transport dynamics have been addressed in this thesis, there is still room for further exploration. In this study, the spectral bandwidth of the THz spectrum is limited to $1.5 \mathrm{THz}$. The spectral bandwidth of the $\mathrm{THz}$ generation could be increased by using different generation crystals or pumping with different wavelengths. By Broadening the $\mathrm{THz}$ bandwidth beyond $1.5 \mathrm{THz}$, we can identify the resonant spectral features. Also, in THz-TDS measurements, we could not identify electron and hole concentrations separately. Similar to the Van-der-Paw method, if we apply an external magnetic field in the THz-TDS, it is possible to distinguish the conductivities of minority and majority charge carriers independently. Also, the backscattering coefficient in the Drude-smith model has not been directly concretely related to microscopic mechanisms. To understand the physical origin, this can be evaluated in terms of tensor elements. 
We have studied the excited state carrier dynamics by photoexciting the sample with 400 $\mathrm{nm}$. Performing excitation wavelength dependence experiments will provide further insights into dynamical processes. We have carried out photoluminescence experimental to study radiative emission from samples. However, photoluminescence was not observed in samples at $300 \mathrm{~K}$. To confirm this observation, the photoluminescence experiment needs to be formed at lower temperatures. Additionally, the thicknesses of chalcopyrite samples $(500 \mu m)$ are much greater than the penetration depths of the sample. This will result in an inhomogeneous excitation. To overcome this issue, thin films can be deposited on a suitable substrate. 


\section{Appendix A}

\section{A.1 Transient Absorption Spectroscopy on Carbon Nitride Crystals}

Optical pump-probe spectroscopy is an advanced technique for the investigation of excitedstate carrier dynamics on short timescales. Compared to the TRTS, an optical pulse is used as a probe in this technique. Here we have studied ultrafast carrier dynamics of carbon nitride (A-g$\left.\mathrm{C}_{3} \mathrm{~N}_{4}\right)$ and crystalline carbon nitride crystals $\left(\mathrm{C}-\mathrm{g}-\mathrm{C}_{3} \mathrm{~N}_{4}\right)$ using optical pump-probe spectroscopy.

Carbon Nitride $\left(\mathrm{g}-\mathrm{C}_{3} \mathrm{~N}_{4}\right)$ crystals are semiconductor photocatalysts that show superior photocatalytic performances. C-g- $\mathrm{C}_{3} \mathrm{~N}_{4}$ powder was synthesized via a two-step heating method using $\mathrm{NH}_{4} \mathrm{SCN}$ as the precursor and $\mathrm{A}-\mathrm{g}-\mathrm{C}_{3} \mathrm{~N}_{4}$ was synthesized by annealing conventionally bulk g- $\mathrm{C}_{3} \mathrm{~N}_{4}$ powder [193]. To perform pump-probe measurements, $\mathrm{C}_{3} \mathrm{~N}_{4}$ powders were mixed with $\mathrm{KBr}$ into 1:20 ration and ground for $10 \mathrm{~min}$. The resulting mixtures were then pressed into pellets.

The thickness of the crystalline $\mathrm{g}-\mathrm{C}_{3} \mathrm{~N}_{4}$ and amorphous $\mathrm{g}-\mathrm{C}_{3} \mathrm{~N}_{4}$ are, $1.76 \times 10^{-2} \mathrm{~cm}^{-1}$ and $2.24 \times 10^{-2} \mathrm{~cm}^{-1}$ respectively. Estimated absorption coefficients at the probe energy for crystalline $\mathrm{g}-\mathrm{C}_{3} \mathrm{~N}_{4}$, and amorphous $\mathrm{g}-\mathrm{C}_{3} \mathrm{~N}_{4}$ are $434.5 \mathrm{~cm}^{-1}$ and $345.2 \mathrm{~cm}^{-1}$ respectively.

Transient absorption measurements on amorphous carbon nitride $\left(\mathrm{A}-\mathrm{g}-\mathrm{C}_{3} \mathrm{~N}_{4}\right)$ and crystalline carbon nitride crystals $\left(\mathrm{C}-\mathrm{g}-\mathrm{C}_{3} \mathrm{~N}_{4}\right)$ are carried out using optical pump-probe spectroscopy as described in section 2.9. Samples are photoexcited with $400 \mathrm{~nm}$ excitation pulse with $290 \mu \mathrm{m}$ spot size and probe with $800 \mathrm{~nm}$ pulse with $90 \mu \mathrm{m}$ spot size.

Figure A.1 shows normalized transient decays of the (a) C-g- $\mathrm{C}_{3} \mathrm{~N}_{4}$ (b) A-g- $\mathrm{C}_{3} \mathrm{~N}_{4}$ at various pump powers. The observed increase in transient signal strength with increasing pump power is due to increased carrier concentration at higher powers. Figure 5.3 (b) shows the transient absorption signal for $\mathrm{C}-\mathrm{g}-\mathrm{C}_{3} \mathrm{~N}_{4}$ and $\mathrm{A}-\mathrm{g}-\mathrm{C}_{3} \mathrm{~N}_{4}$ at $0.3 \mathrm{~mW}$ pump power. $\mathrm{A}-\mathrm{g}-\mathrm{C}_{3} \mathrm{~N}_{4}$ sample has higher absorption compared to $\mathrm{C}-\mathrm{g}-\mathrm{C}_{3} \mathrm{~N}_{4}$ sample. Each decay of figure 5.4 was fit by the threeexponential model and each decay time was assigned to a different recombination mechanism. Table A.1 shows the fit parameters for $0.3 \mathrm{~mW}$. We attributed fast component $\left(\tau_{1} \sim p s\right)$ to the electron trapping and transfer, medium component $\left(\tau_{2} \sim p s\right)$ to the Auger recombination and slow decay time $\left(\tau_{3} \sim n s\right)$ to the radiative recombination and nonradiative relaxation. While these mechanisms typically dominate, other mechanisms may also occur or modify the carrier dynamics. 
The $\mathrm{C}-\mathrm{g}-\mathrm{C}_{3} \mathrm{~N}_{4}$ exhibits recombination rates for all processes that are slower compared to that of A-g- $\mathrm{C}_{3} \mathrm{~N}_{4}$, thus resulting in a longer lifetime of the charge carriers
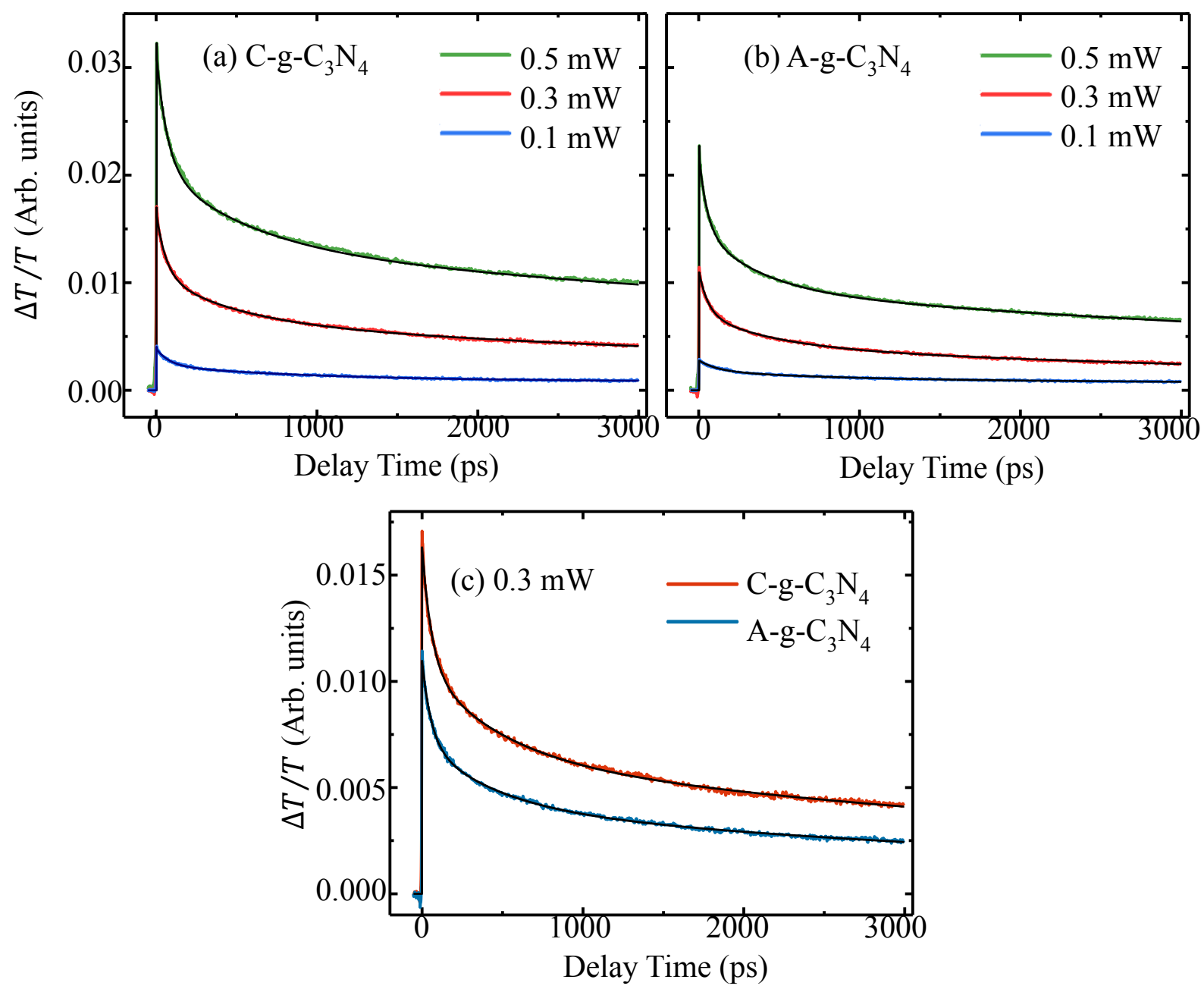

Figure A.1. (a). Normalized transient absorption decay for $\mathrm{C}-\mathrm{g}-\mathrm{C}_{3} \mathrm{~N}_{4}$ at various pump powers. (b). Normalized transient absorption decay for $\mathrm{A}-\mathrm{g}-\mathrm{C}_{3} \mathrm{~N}_{4}$ at various pump powers. (c). Normalized transient absorption decay for $\mathrm{C}-\mathrm{g}-\mathrm{C}_{3} \mathrm{~N}_{4}$ and $\mathrm{AC}-\mathrm{g}-\mathrm{C}_{3} \mathrm{~N}_{4}$ at $0.3 \mathrm{~mW}$ pump power fitted with multiexponential function.

Table A.1: Fit parameters for multiple recombination modeling of C-g- $\mathrm{C}_{3} \mathrm{~N}_{4}$ and A-g- $\mathrm{C}_{3} \mathrm{~N}_{4}$

\begin{tabular}{lcccccc}
\hline Sample & $A_{1}$ & $A_{2}$ & $A_{3}$ & $\tau_{1}(\mathrm{ps})$ & $\tau_{2}(\mathrm{ps})$ & $\tau_{3}(\mathrm{ps})$ \\
\hline $\mathrm{C}-\mathrm{g}-\mathrm{C}_{3} \mathrm{~N}_{4}$ & 0.0055 & 0.0045 & 0.0063 & 59.96 & 490.18 & 6919.22 \\
$\mathrm{~A}-\mathrm{g}-\mathrm{C}_{3} \mathrm{~N}_{4}$ & 0.0038 & 0.0032 & 0.0041 & 56.25 & 446.36 & 5871.10 \\
\hline
\end{tabular}


As described in section 4.4, the multiexponential method only valid in the low carrier density regime and we need to know the recombination mechanisms in advance. To overcome these issues, the Inversion analysis technique was applied to experimental transients. Figure A.2 (a) shows the inverted transient-absorption decay as a function of carrier density for $\mathrm{C}-\mathrm{g}-\mathrm{C}_{3} \mathrm{~N}_{4}$ and A-g- $\mathrm{C}_{3} \mathrm{~N}_{4}$ on a $\log -\log$ plot. The slope of the plot corresponds to the order of nonlinear relaxation mechanisms. As shown in figure A.2 (a) at low carrier density $\left(<2 \times 10^{16} \mathrm{~cm}^{-3}\right)$ slopes of the inverted transient plot are 1 and 2 . This reveals that the dominant recombination mechanisms are radiative and non-radiative relaxations in this regime. At medium carrier density regime, $\left(2.5 \times 10^{16} \mathrm{~cm}^{-3}-3.8 \times 10^{16} \mathrm{~cm}^{-3}\right)$ the slope of the plot is 3.This reveals that the carrier relaxation is due to Auger recombination in the medium carrier density regime. A-g- $\mathrm{C}_{3} \mathrm{~N}_{4}$ sample shows an upward shift in the position of the slope compared to $\mathrm{C}-\mathrm{g}-\mathrm{C}_{3} \mathrm{~N}_{4}$ sample indicating that the $\mathrm{C}-\mathrm{g}-\mathrm{C}_{3} \mathrm{~N}_{4}$ exhibits slower relaxation for all processes than that of the A-g- $\mathrm{C}_{3} \mathrm{~N}_{4}$.
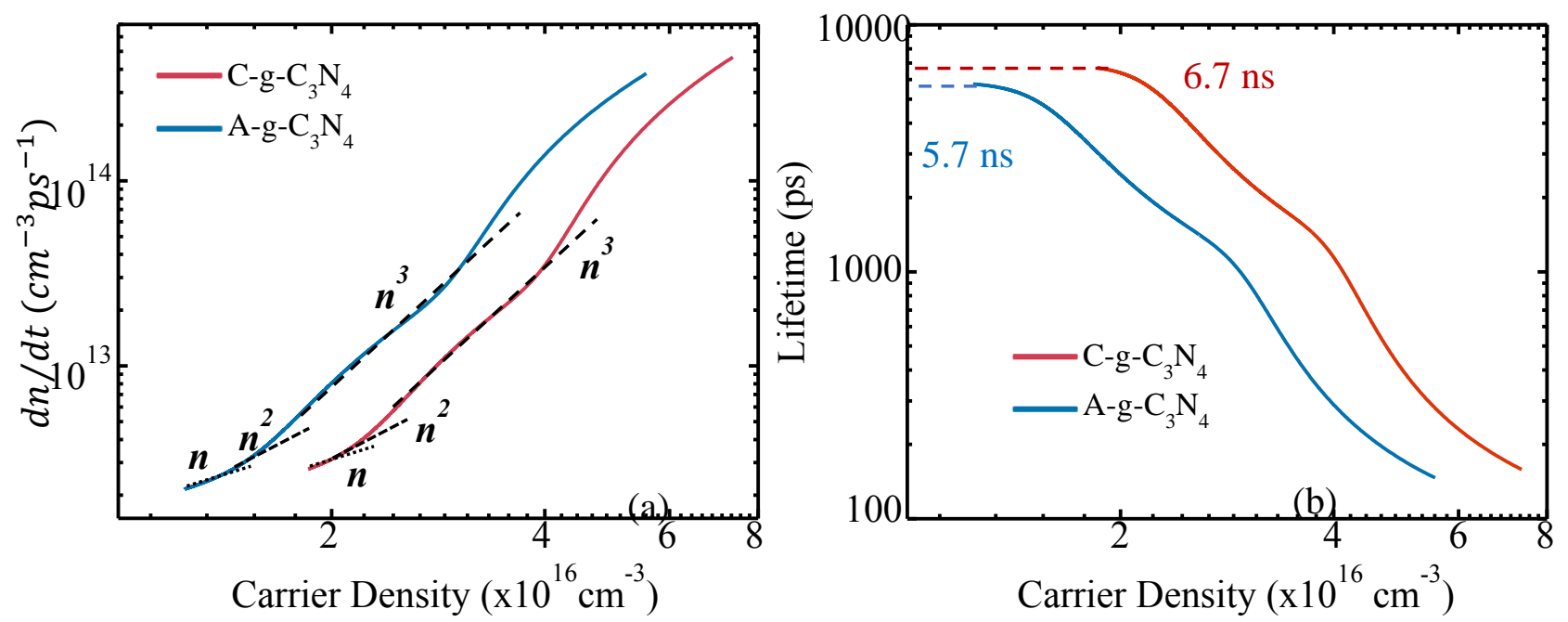

Figure A.2 (a) The inverted spectra showing the relaxation kinetics of C-g- $\mathrm{C}_{3} \mathrm{~N}_{4}$ and $\mathrm{A}-\mathrm{g}-\mathrm{C}_{3} \mathrm{~N}_{4}$ (b) Instantaneous lifetime as a function of carrier density for two samples.

Figure A.2 (b) shows the instantaneous lifetime for the two samples calculated using equation 5.4. It can be observed that the lifetime is shorter at higher carrier densities and it flattens off at low carrier densities. C-g- $\mathrm{C}_{3} \mathrm{~N}_{4}$ sample has a lifetime of $6.7 \mathrm{~ns}$ extrapolated at low carrier densities whereas A-g- $\mathrm{C}_{3} \mathrm{~N}_{4}$ has a shorter lifetime of $5.7 \mathrm{~ns}$. The instantaneous lifetime results also 
confirmed that the $\mathrm{C}-\mathrm{g}-\mathrm{C}_{3} \mathrm{~N}_{4}$ exhibits decay rates for all recombination mechanisms that are slower than that of the A-g- $\mathrm{C}_{3} \mathrm{~N}_{4}$, thus resulting in a longer lifetime of the charge carriers. This study shows that by turning the crystallinity of the $\mathrm{g}_{-} \mathrm{C}_{3} \mathrm{~N}_{4}$, radiative and non-radiative relaxations of the charge carriers can be minimized, resulting in a longer lifetime and eventually a high photocatalytic performance. 


\section{References}

1. Y. R. Nosov, "History of optoelectronics: general review," in V. G. Inozemtsev and V. A. Shilin, eds. (2002), pp. 20-27.

2. B. Lojek, History of Semiconductor Engineering (Springer-Verlag, 2007).

3. H. R. Huff, "John Bardeen and transistor physics," in AIP Conference Proceedings (AIP, 2001), Vol. 550, pp. 3-32.

4. M. I. Nathan, W. P. Dumke, G. Burns, F. H. Dill, and G. Lasher, "STIMULATED EMISSION OF RADIATION FROM GaAs p-n JUNCTIONS," Appl. Phys. Lett. 1, 62-64 (1962).

5. J. Hecht, "Short history of laser development," OE 49, 091002 (2010).

6. Z. Liu, W. Ma, and X. Ye, "Chapter 2 - Shape control in the synthesis of colloidal semiconductor nanocrystals," in Anisotropic Particle Assemblies, N. Wu, D. Lee, and A. Striolo, eds. (Elsevier, 2018), pp. 37-54.

7. O. Wada, "Optoelectronic integration based on GaAs material," Opt Quant Electron 20, 441474 (1988).

8. S. J. Sweeney and J. Mukherjee, "Optoelectronic Devices and Materials," in Springer Handbook of Electronic and Photonic Materials, S. Kasap and P. Capper, eds., Springer Handbooks.

9. E. M. Clarke and R. Murray, "Chapter 3 - Optical Properties of In(Ga)As/GaAs Quantum Dots for Optoelectronic Devices," in Handbook of Self Assembled Semiconductor Nanostructures for Novel Devices in Photonics and Electronics, M. Henini, ed. (Elsevier, 2008), pp. 84-131.

10.S. Mokkapati and C. Jagadish, "III-V compound SC for optoelectronic devices," Materials Today 12, 22-32 (2009).

11.J. Cai, H. Opper, R. Basilica, B. Garber, and G. W. Taylor, "GaAs based monolithic optoelectronic device integration technology," in Nanophotonics and Macrophotonics for Space Environments IV (International Society for Optics and Photonics, 2010), Vol. 7817, p. $78170 \mathrm{P}$.

12.X. Duan, Y. Huang, Y. Cui, J. Wang, and C. M. Lieber, "Indium phosphide nanowires as building blocks for nanoscale electronic and optoelectronic devices," Nature 409, 66-69 (2001).

13.F. Zafar and A. Iqbal, "Indium phosphide nanowires and their applications in optoelectronic devices," Proceedings of the Royal Society A: Mathematical, Physical and Engineering Sciences 472, 20150804 (2016).

14.A. G. Milnes and A. Y. Polyakov, "Indium arsenide: a semiconductor for high speed and electro-optical devices," Materials Science and Engineering: B 18, 237-259 (1993). 
15. H. J. Joyce, Q. Gao, J. Wong-Leung, Y. Kim, H. H. Tan, and C. Jagadish, "Tailoring GaAs, InAs, and InGaAs Nanowires for Optoelectronic Device Applications," IEEE Journal of Selected Topics in Quantum Electronics 17, 766-778 (2011).

16. J. C. Johnson, H.-J. Choi, K. P. Knutsen, R. D. Schaller, P. Yang, and R. J. Saykally, "Single gallium nitride nanowire lasers," Nature Materials 1, 106-110 (2002).

17. N. Aggarwal and G. Gupta, "Enlightening gallium nitride-based UV photodetectors," J. Mater. Chem. C 8, 12348-12354 (2020).

18. M. A. Weissflog, M. Cai, M. Parry, M. Rahmani, L. Xu, A. N. Fedotova, G. Marino, M. Lysevych, H. Tan, C. Jagadish, A. Miroshnichenko, G. Leo, A. A. Sukhorukov, F. Setzpfandt, T. Pertsch, I. Staude, and D. N. Neshev, "Non-Degenerate Nonlinear Frequency Mixing in (110)-Grown GaAs Nanoresonators," in 2020 Conference on Lasers and Electro-Optics (CLEO) (2020), pp. 1-2.

19.A. I. Kuznetsov, A. E. Miroshnichenko, M. L. Brongersma, Y. S. Kivshar, and B. Luk'yanchuk, "Optically resonant dielectric nanostructures," Science 354, (2016).

20. R. C. Miller, G. D. Boyd, and A. Savage, "NONLINEAR OPTICAL INTERACTIONS IN $\mathrm{LiNbO}_{3}$ WITHOUT DOUBLE REFRACTION," Appl. Phys. Lett. 6, 77-79 (1965).

21. A. Feisst and P. Koidl, "Current induced periodic ferroelectric domain structures in $\mathrm{LiNbO}_{3}$ applied for efficient nonlinear optical frequency mixing," Appl. Phys. Lett. 47, 1125-1127 (1985).

22. W. Liu, L. Zhu, and C. Fang, "Observation of sum-frequency-generation-induced cascaded four-wave mixing using two crossing femtosecond laser pulses in a $0.1 \mathrm{~mm}$ beta-barium-borate crystal," Opt. Lett., 37, 3783-3785 (2012).

23. J. G. Haub, M. J. Johnson, and B. J. Orr, "Spectroscopic and nonlinear-optical applications of a tunable $\beta$-barium borate optical parametric oscillator," J. Opt. Soc. Am. B, 10, 1765-1777 (1993).

24. D. N. Nikogosyan, "Beta barium borate (BBO)," Appl. Phys. A 52, 359-368 (1991).

25. E. S. Polzik and H. J. Kimble, "Frequency doubling with $\mathrm{KNbO}_{3}$ in an external cavity," Opt. Lett.,16, 1400-1402 (1991).

26. W. J. Alford and A. V. Smith, "Wavelength variation of the second-order nonlinear coefficients of $\mathrm{KNbO}_{3}, \mathrm{KTiOPO}_{4}, \mathrm{KTiOAsO}_{4}, \mathrm{LiNbO}_{3}, \mathrm{LiIO}_{3}, \beta-\mathrm{BaB}_{2} \mathrm{O}_{4}, \mathrm{KH}_{2} \mathrm{PO}_{4}$, and $\mathrm{LiB}_{3} \mathrm{O}_{5}$ crystals: a test of Miller wavelength scaling," J. Opt. Soc. Am. B, 18, 524-533 (2001).

27. R. G. Walker, N. Cameron, Y. Zhou, and S. Clements, "Electro-optic modulators for space using gallium arsenide," in International Conference on Space Optics - ICSO 2016 (International Society for Optics and Photonics, 2017), Vol. 10562, p. 105621A.

28.F. K. Reinhart, "Reverse-Biased Gallium Phosphide Diodes as High-Frequency Light Modulators," Journal of Applied Physics 39, 3426-3434 (1968). 
29. J. D. Rowley, J. K. Pierce, A. T. Brant, L. E. Halliburton, N. C. Giles, P. G. Schunemann, and A. D. Bristow, "Broadband terahertz pulse emission from $\mathrm{ZnGeP}_{2}$," Optics Letters 37, 788 (2012).

30. J. D. Rowley, D. A. Bas, K. T. Zawilski, P. G. Schunemann, and A. D. Bristow, "Terahertz emission from $\mathrm{ZnGeP}_{2}$ : phase-matching, intensity, and length scalability," J. Opt. Soc. Am. B, 30, 2882-2888 (2013).

31.G. Marchev, A. Tyazhev, V. Petrov, P. G. Schunemann, K. T. Zawilski, G. Stöppler, and M. Eichhorn, "Optical parametric generation in $\mathrm{CdSiP}_{2}$ at $6.125 \mu \mathrm{m}$ pumped by $8 \mathrm{~ns}$ long pulses at 1064 nm," Opt. Lett.,37, 740-742 (2012).

32. V. Kemlin, B. Boulanger, V. Petrov, P. Segonds, B. Ménaert, P. G. Schunneman, and K. T. Zawilski, "Nonlinear, dispersive, and phase-matching properties of the new chalcopyrite $\mathrm{CdSiP}_{2}$ [Invited]," Opt. Mater. Express, 1, 1292-1300 (2011).

33. J. L. Shay and J. H. Wernick, Ternary Chalcopyrite Semiconductors (Pergamon, Oxford, 1975)

34. H. Hahn, G. Frank, W. Klingler, A.-D. Meyer, and G. Störger, "Untersuchungen über ternäre Chalkogenide. V. Über einige ternäre Chalkogenide mit Chalkopyritstruktur," Zeitschrift für anorganische und allgemeine Chemie 271, 153-170 (1953).

35. C. H. L. Goodman, "A New Group of Compounds with Diamond type (Chalcopyrite) Structure," Nature 179, 828-829 (1957).

36. C. H. L. Goodman, "The prediction of semiconducting properties in inorganic compounds," Journal of Physics and Chemistry of Solids 6, 305-314 (1958).

37. G. A. Medvedkin and V. G. Voevodin, "Magnetic and optical phenomena in nonlinear optical crystals $\mathrm{ZnGeP}_{2}$ and $\mathrm{CdGeP}_{2}$," J. Opt. Soc. Am. B, JOSAB 22, 1884-1898 (2005).

38. V. M. Novotortsev, A. V. Kochura, and S. F. Marenkin, "New ferromagnetics based on manganese-alloyed chalcopyrites $\mathrm{A}_{\mathrm{II}} \mathrm{B}_{\mathrm{IV}} \mathrm{C}_{2} \mathrm{~V}$," Inorg Mater 46, 1421-1436 (2010).

39. R. Gautam, P. Singh, S. Sharma, S. Kumari, and A. S. Verma, "Structural, electronic, optical, elastic and thermal properties of $\mathrm{CdSnP}_{2}$ with the application in solar cell devices," Superlattices and Microstructures 85, 859-871 (2015).

40. Y. X. Fan, R. C. Eckardt, R. L. Byer, R. K. Route, and R. S. Feigelson, "AgGaS 2 infrared parametric oscillator," Appl. Phys. Lett. 45, 313-315 (1984).

41. S. C. Kumar, M. Jelínek, M. Baudisch, K. T. Zawilski, P. G. Schunemann, V. Kubeček, J. Biegert, and M. Ebrahim-Zadeh, "Tunable, high-energy, mid-infrared, picosecond optical parametric generator based on $\mathrm{CdSiP}_{2}$," Optics Express 20, 15703 (2012).

42. R. S. Feigelson and R. K. Route,"Recent Developments in the Growth Of Chalcopyrite Crystals For Nonlinear Infrared Applications," Opt. Eng. 1987, 26, 113-119. 
43. J.-B. Dherbecourt, A. Godard, M. Raybaut, J.-M. Melkonian, and M. Lefebvre, "Picosecond synchronously pumped $\mathrm{ZnGeP}_{2}$ optical parametric oscillator," Opt. Lett., 35, 2197-2199 (2010).

44. P.-H. Chuang, C. C. Lin, and R.-S. Liu, "Emission-Tunable CuInS 2 /ZnS Quantum Dots: Structure, Optical Properties, and Application in White Light-Emitting Diodes with High Color Rendering Index," ACS Appl. Mater. Interfaces 6, 15379-15387 (2014).

45. M. C. Ohmer and R. Pandey, "Emergence of Chalcopyrites as Nonlinear Optical Materials," MRS Bulletin 23, 16-22 (1998).

46. V. Petrov, "New applications of chalcopyrite crystals in nonlinear optics," physica status solidi c 14, 1600161 (2017).

47. H. P. Piyathilaka, R. Sooriyagoda, V. Dewasurendra, M. B. Johnson, K. T. Zawilski, P. G. Schunemann, and A. D. Bristow, "Terahertz generation by optical rectification in chalcopyrite crystals $\mathrm{ZnGeP}_{2}, \mathrm{CdGeP}_{2}$ and $\mathrm{CdSiP}_{2}$," Optics Express 27, 16958 (2019).

48. B. N. Carnio, P. G. Schunemann, K. T. Zawilski, and A. Y. Elezzabi, "Generation of broadband terahertz pulses via optical rectification in a chalcopyrite $\mathrm{CdSiP}_{2}$ crystal," Opt. Lett., 42, 39203923 (2017).

49. J. D. Rowley, J. K. Wahlstrand, K. T. Zawilski, P. G. Schunemann, N. C. Giles, and A. D. Bristow, "Terahertz generation by optical rectification in uniaxial birefringent crystals," Opt. Express 20, 16968 (2012).

50. B. N. Carnio, K. T. Zawilski, P. G. Schunemann, and A. Y. Elezzabi, "Terahertz birefringence and absorption of a chalcopyrite $\mathrm{CdSiP}_{2}$ crystal," Applied Physics Letters 111, 221103 (2017).

51. B. N. Carnio, S. R. Greig, C. J. Firby, K. T. Zawilski, P. G. Schunemann, and A. Y. Elezzabi, "Terahertz electro-optic detection using a $\langle 012\rangle$-cut chalcopyrite $\mathrm{ZnGeP}_{2}$ crystal," Appl. Phys. Lett. 108, 261109 (2016).

52. W. Feng, D. Xiao, J. Ding, and Y. Yao, "Three-dimensional topological insulators in I-III-VI 2 and II-IV-V 2 chalcopyrite semiconductors," Phys Rev Lett 106, 016402 (2011).

53. P. G. Schunemann, K. T. Zawilski, L. A. Pomeranz, D. J. Creeden, and P. A. Budni, "Advances in nonlinear optical crystals for mid-infrared coherent sources," Journal of the Optical Society of America B 33, D36 (2016).

54. G. Boyd, E. Buehler, F. Storz, and J. Wernick, "Linear and nonlinear optical properties of ternary $\mathrm{A}_{\mathrm{II}} \mathrm{B}_{\mathrm{IV}} \mathrm{C}^{2} \mathrm{v}$ chalcopyrite semiconductors," IEEE Journal of Quantum Electronics 8, 419426 (1972).

55. P. G. Schunemann and T. M. Pollak, "Method for growing crystals," U. S. patent 5,611,856 (March 18, 1997).

56 P. G. Schunemann and T. M. Pollak, "Ultralow gradient HGF-grown ZnGeP2 and $\mathrm{CdGeAs}_{2}$ and their optical properties," MRS Bull. 23 (7), 23-27 (1998) 
57. A. J. Miller and W. Clark, "ELECTRICAL PROPERTIES OF ZnGeP 2 AND CdGeP2," Journal de Physique Colloques 36, C3-73-C3-75 (1975).

58. J. Xiao, Z. He, S. Zhu, B. Chen, and G. Jiang, "Hybrid functional study of structural, electronic, bonding and optical properties of $\mathrm{CdSiP}_{2}$," Computational Materials Science 117, 472-477 (2016).

59. "Electronic structure and optical properties of ternary $\mathrm{CdXP}_{2}$ semiconductors $(\mathrm{X}=\mathrm{Si}, \mathrm{Ge}$ and Sn) under pressure," Physica B: Condensed Matter 349, 181-191 (2004).

60. Z. He, B. Zhao, S. Zhu, B. Chen, H. Hou, Y. Yu, and L. Xie, "Theoretical investigation of electronic structure, elastic and thermodynamic properties of chalcopyrite $\mathrm{CdSiP}_{2}$," Computational Materials Science 72, 26-31 (2013).

61. S. K. Tripathy and V. Kumar, "Electronic, elastic and optical properties of $\mathrm{ZnGeP}_{2}$ semiconductor under hydrostatic pressures," Materials Science and Engineering: B 182, 52-58 (2014).

62. S. Zhang, D. Zeng, H. Hou, and Y. Yu, "First-principles prediction on elastic anisotropic, optical, lattice dynamical properties of $\mathrm{ZnGeP}_{2}$," Indian Journal of Physics (2019).

63. E. M. Golden, N. C. Giles, E. Maniego, F. K. Hopkins, K. T. Zawilski, P. G. Schunemann, and L. E. Halliburton, "Identification of native defects (vacancies and antisites) in $\mathrm{CdSiP}_{2}$ crystals," Journal of Applied Physics 118, 185702 (2015).

64. N. C. Giles, L. E. Halliburton, S. Yang, X. Yang, A. T. Brant, N. C. Fernelius, P. G. Schunemann, and K. T. Zawilski, "Optical and EPR study of point defects in CdSiP2 crystals," Journal of Crystal Growth 312, 1133-1137 (2010).

65. S. D. Setzler, P. G. Schunemann, T. M. Pollak, M. C. Ohmer, J. T. Goldstein, F. K. Hopkins, K. T. Stevens, L. E. Halliburton, and N. C. Giles, "Characterization of defect-related optical absorption in ZnGeP2," Journal of Applied Physics 86, 6677-6681 (1999).

66. S. D. Setzler, N. C. Giles, L. E. Halliburton, P. G. Schunemann, and T. M. Pollak, "Electron paramagnetic resonance of a cation antisite defect in $\mathrm{ZnGeP}_{2}$," Applied Physics Letters 74, 1218-1220 (1999).

67. C. Wang, J. Sun, H. Gou, S. Wang, J. Zhang, and X. Tao, "Intrinsic defects and their effects on the optical properties in the nonlinear optical crystal $\mathrm{CdSiP}_{2}$ : a first-principles study," Physical Chemistry Chemical Physics 19, 9558-9565 (2017).

68. M. Bettini and A. Miller, "Optical Phonons in $\mathrm{ZnGeP}_{2}$ and $\mathrm{CdGeP}_{2}$," physica status solidi (b) 66, 579-586 (1974).

69. S. Shirakata, "Hydrostatic Pressure Dependence of Raman Spectra in CdSiP2 Crystal," Jpn. J. Appl. Phys. 27, 2113 (1988).

70. K. Zhong, C. Liu, M. Wang, J. Shi, B. Kang, Z. Yuan, J. Li, D. Xu, W. Shi, and J. Yao, "Linear optical properties of ZnGeP_2 in the terahertz range," Opt. Mater. Express 7, 3571 (2017). 
71. B. N. Carnio, K. T. Zawilski, P. G. Schunemann, and A. Y. Elezzabi, "Birefringence, absorption, and optical rectification of a chalcopyrite $\mathrm{CdSiP} 2$ crystal in the terahertz frequency regime," in Terahertz, RF, Millimeter, and Submillimeter-Wave Technology and Applications XI (International Society for Optics and Photonics, 2018), Vol. 10531, p. 105311P.

72. M. R. Ferdinandus, M. R. Ferdinandus, J. J. Gengler, J. J. Gengler, K. L. Averett, K. L. Averett, K. T. Zawilski, P. G. Schunemann, and C. M. Liebig, "Nonlinear optical measurements of $\mathrm{CdSiP}_{2}$ at near and mid-infrared wavelengths," Opt. Mater. Express, 10, 2066-2074 (2020).

73. R. Sooriyagoda, H. P. Piyathilaka, V. Dewasurendra, M. B. Johnson, K. T. Zawilski, P. G. Schunemann, and A. D. Bristow, "Terahertz generation by optical rectification in chalcopyrite crystals $\mathrm{ZnGeP}_{2}, \mathrm{CdGeP}_{2}$ and $\mathrm{CdSiP}_{2}$," in Frontiers in Optics + Laser Science APS/DLS (2019), Paper JW4A.37.

74. P. G. Schunemann, K. T. Zawilski, T. M. Pollak, V. Petrov, and D. E. Zelmon, "CdSiP 2 : a new nonlinear optical crystal for 1- and 1.5-micron-pumped mid-IR generation," in Advanced SolidState Photonics (2009), Paper TuC6

75. E. Hecht, Óptica, 3rd ed. (Addison-Wesley, Madrid, 2000).

76. J. D. Jackson, Classical Electrodynamics (Wiley, New York, 1962).

77. P. Vasa, "Strong Light-Matter Interaction," in Digital Encyclopedia of Applied Physics (American Cancer Society, 2019), pp. 1-30.

78. Kittel, C., 1963, Quantum Theory of Solids (Wiley, New York)

79. A. Othonos, "Probing ultrafast carrier and phonon dynamics in semiconductors," Journal of Applied Physics 83, 1789-1830 (1998).

80. J. Shah, "Hot electrons and phonons under high intensity photoexcitation of semiconductors," Solid-State Electronics 21, 43-50 (1978).

81. T. H. Maiman, "Stimulated Optical Radiation in Ruby," Nature 187, 493-494 (1960).

82.T.F. Johnston,Jr., F.J. Duarte, in Encyclopedia of Physical Science and Technology (Third Edition), 2003

83.H. A. Haus, "Mode-locking of lasers," IEEE Journal of Selected Topics in Quantum Electronics 6, 1173-1185 (2000).

84. H. W. Mocker and R. J. Collins, "Mode competition and self-locking effects in a q-switched ruby laser," Appl. Phys. Lett. 7, 270-273 (1965).

85. A. J. DeMaria, D. A. Stetser, and H. Heynau, "Self mode-locking of lasers with saturable absorbers," Appl. Phys. Lett. 8, 174-176 (1966).

86. C. V. Shank and E. P. Ippen, "Subpicosecond kilowatt pulses from a mode-locked cw dye laser," Appl. Phys. Lett. 24, 373-375 (1974). 
87. J. L. Levy and R. C. Mayer, "Solutions for the laser rate equations for cavity-dumping with pulse position modulation," Mathematical and Computer Modelling 12, 919-923 (1989).

88. P. F. Moulton, "Spectroscopic and laser characteristics of $\mathrm{Ti}: \mathrm{Al}_{2} \mathrm{O}_{3}$," J. Opt. Soc. Am. B,3, 125-133 (1986).

89. I. V. Yakovlev, "Stretchers and compressors for ultra-high power laser systems," Quantum Electron. 44, 393 (2014).

90. Y. Shi, Q. Zhou, C. Zhang, and B. Jin, "Ultrafast high-field carrier transport in GaAs measured by femtosecond pump-terahertz probe spectroscopy," Appl. Phys. Lett. 93, 121115 (2008).

91. E. Serpetzoglou, I. Konidakis, G. Kakavelakis, T. Maksudov, E. Kymakis, and E. Stratakis, "Improved Carrier Transport in Perovskite Solar Cells Probed by Femtosecond Transient Absorption Spectroscopy," ACS Appl. Mater. Interfaces 9, 43910-43919 (2017).

92. G. Segschneider, F. Jacob, T. Löffler, H. G. Roskos, S. Tautz, P. Kiesel, and G. Döhler, "Freecarrier dynamics in low-temperature-grown GaAs at high excitation densities investigated by time-domain terahertz spectroscopy," Phys. Rev. B 65, 125205 (2002).

93. S. Xu, J. Yang, H. Jiang, F. Su, and Z. Zeng, "Transient photoconductivity and free carrier dynamics in a monolayer WS2 probed by time resolved Terahertz spectroscopy," Nanotechnology 30, 265706 (2019).

94. M. C. Beard, J. L. Blackburn, and M. J. Heben, "Photogenerated Free Carrier Dynamics in Metal and Semiconductor Single-Walled Carbon Nanotube Films," Nano Lett. 8, 4238-4242 (2008).

95. A. J. Fischer, W. Shan, G. H. Park, J. J. Song, D. S. Kim, D. S. Yee, R. Horning, and B. Goldenberg, "Femtosecond four-wave-mixing studies of nearly homogeneously broadened excitons in GaN," Phys. Rev. B 56, 1077-1080 (1997).

96. T. Meier, V. Chernyak, and S. Mukamel, "Multiple Exciton Coherence Sizes in Photosynthetic Antenna Complexes viewed by Pump-Probe Spectroscopy," J. Phys. Chem. B 101, 7332-7342 (1997).

97. H. Wang, J. H. Strait, P. A. George, S. Shivaraman, V. B. Shields, M. Chandrashekhar, J. Hwang, F. Rana, M. G. Spencer, C. S. Ruiz-Vargas, and J. Park, "Ultrafast relaxation dynamics of hot optical phonons in graphene," Appl. Phys. Lett. 96, 081917 (2010).

98. M. Hase, K. Mizoguchi, H. Harima, S. Nakashima, and K. Sakai, "Dynamics of coherent phonons in bismuth generated by ultrashort laser pulses," Phys. Rev. B 58, 5448-5452 (1998).

99. X. H. Zhang, H. C. Guo, A. M. Yong, J. D. Ye, S. T. Tan, and X. W. Sun, "Terahertz dielectric response and optical conductivity of n-type single-crystal $\mathrm{ZnO}$ epilayers grown by metalorganic chemical vapor deposition," Journal of Applied Physics 107, 033101 (2010). 
100. W. Qiao, K. Yang, A. Thoma, and T. Dekorsy, "Dielectric Relaxation of $\mathrm{HCl}$ and $\mathrm{NaCl}$ Solutions Investigated by Terahertz Time-Domain Spectroscopy," J Infrared Milli Terahz Waves 33, 1029-1038 (2012).

101. X. Zou, J. Luo, D. Lee, C. Cheng, D. Springer, S. K. Nair, S. A. Cheong, H. J. Fan, and E. E. M. Chia, "Temperature-dependent terahertz conductivity of tin oxide nanowire films," J. Phys. D: Appl. Phys. 45, 465101 (2012).

102. Y.-S. Lee, Principles of Terahertz Science and Technology (Springer US, 2009).

103. E. Cinquanta, D. Meggiolaro, S. G. Motti, M. Gandini, M. J. P. Alcocer, Q. A. Akkerman, C. Vozzi, L. Manna, F. De Angelis, A. Petrozza, and S. Stagira, "Ultrafast THz Probe of Photoinduced Polarons in Lead-Halide Perovskites," Phys. Rev. Lett. 122, 166601 (2019).

104. A. C. Das, S. Bhattacharya, M. Jewariya, S. S. Prabhu, K. C. Mandal, T. Ozaki, and P. K. Datta, "Identification of Combination Phonon Modes in Pure and Doped GaSe Crystals by THz Spectroscopy," IEEE Journal of Selected Topics in Quantum Electronics 23, 1-7 (2017).

105. M. C. Beard, G. M. Turner, and C. A. Schmuttenmaer, "Transient photoconductivity in GaAs as measured by time-resolved terahertz spectroscopy," Phys. Rev. B 62, 15764-15777 (2000).

106. J. B. Baxter and C. A. Schmuttenmaer, "Conductivity of ZnO Nanowires, Nanoparticles, and Thin Films Using Time-Resolved Terahertz Spectroscopy," J. Phys. Chem. B 110, 2522925239 (2006).

107. J. B. Baxter and C. A. Schmuttenmaer, "Carrier dynamics in bulk ZnO. II. Transient photoconductivity measured by time-resolved terahertz spectroscopy," Phys. Rev. B 80, 235206 (2009).

108. M. Tonouchi, "Cutting-edge terahertz technology," Nature Photonics 1, 97-105 (2007).

109. K. Kawase, Y. Ogawa, Y. Watanabe, and H. Inoue, "Non-destructive terahertz imaging of illicit drugs using spectral fingerprints," Opt. Express, 11, 2549-2554 (2003).

110. R. M. Woodward, B. E. Cole, V. P. Wallace, R. J. Pye, D. D. Arnone, E. H. Linfield, and M. Pepper, "Terahertz pulse imaging in reflection geometry of human skin cancer and skin tissue," Phys. Med. Biol. 47, 3853-3863 (2002).

111. A. Hirata, T. Kosugi, H. Takahashi, R. Yamaguchi, F. Nakajima, T. Furuta, H. Ito, H. Sugahara, Y. Sato, and T. Nagatsuma, "120-GHz-band millimeter-wave photonic wireless link for 10-Gb/s data transmission," IEEE Transactions on Microwave Theory and Techniques 54, 1937-1944 (2006).

112. G. Mourou, C. V. Stancampiano, A. Antonetti, and A. Orszag, "Picosecond microwave pulses generated with a subpicosecond laser-driven semiconductor switch," Appl. Phys. Lett. 39, 295296 (1981).

113. M. Bass, P. A. Franken, J. F. Ward, and G. Weinreich, "Optical Rectification," Phys. Rev. Lett. 9, 446-448 (1962). 
114. J. A. Valdmanis, G. Mourou, and C. W. Gabel, "Picosecond electro-optic sampling system," Appl. Phys. Lett. 41, 211-212 (1982).

115. X. -C. Zhang, Y. Jin, and X. F. Ma, "Coherent measurement of THz optical rectification from electro-optic crystals," Appl. Phys. Lett. 61, 2764-2766 (1992).

116. L. Xu, X. -C. Zhang, and D. H. Auston, "Terahertz beam generation by femtosecond optical pulses in electro-optic materials," Appl. Phys. Lett. 61, 1784-1786 (1992).

117. X. -C. Zhang, X. F. Ma, Y. Jin, T. -M. Lu, E. P. Boden, P. D. Phelps, K. R. Stewart, and C. P. Yakymyshyn, "Terahertz optical rectification from a nonlinear organic crystal," Appl. Phys. Lett. 61, 3080-3082 (1992).

118. Q. Wu and X. -C. Zhang, "Free-space electro-optic sampling of terahertz beams," Appl. Phys. Lett. 67, 3523-3525 (1995).

119. P. U. Jepsen, C. Winnewisser, M. Schall, V. Schyja, S. R. Keiding, and H. Helm, "Detection of THz pulses by phase retardation in lithium tantalate," Phys. Rev. E 53, R3052-R3054 (1996).

120. J. R. Danielson, A. D. Jameson, J. L. Tomaino, H. Hui, J. D. Wetzel, Y.-S. Lee, and K. L. , "Intense narrow band terahertz generation via type-II difference-frequency generation in $\mathrm{ZnTe}$ using chirped optical pulses," Journal of Applied Physics 104, 033111 (2008).

121. L. Hou and W. Shi, "An LT-GaAs Terahertz Photoconductive Antenna With High Emission Power, Low Noise, and Good Stability," IEEE Transactions on Electron Devices 60, 16191624 (2013).

122. H. Zhong, N. Karpowicz, and X.-C. Zhang, "Terahertz emission profile from laser-induced air plasma," Appl. Phys. Lett. 88, 261103 (2006).

123. Boyd, R., 2003, Nonlinear Optics, 2nd ed. (Academic, New York)

124 Shen, Y. R., 1984, The Principles of Nonlinear Optics (Wiley, New York).

125. S. L. Dexheimer, Terahertz Spectroscopy Principles and Applications (CRC Press, 2008).

126. B. N. Carnio, K. T. Zawilski, P. G. Schunemann, and A. Y. Elezzabi, "Optical rectification in a chalcopyrite $\mathrm{AgGaSe} 2$ crystal for broadband terahertz radiation generation," Opt. Lett., 44, 2867-2870 (2019).

127. I. Shoji, T. Kondo, A. Kitamoto, M. Shirane, and R. Ito, "Absolute scale of second-order nonlinear-optical coefficients," J. Opt. Soc. Am. B 14, 2268 (1997).

128. V. Petrov, F. Noack, I. Tunchev, P. Schunemann, and K. Zawilski, "The nonlinear coefficient d 36 of $\mathrm{CdSiP}_{2}$," in P. E. Powers, ed. (2009), p. 71970M.

129. A. Nahata, A. S. Weling, and T. F. Heinz, "A wideband coherent terahertz spectroscopy system using optical rectification and electro-optic sampling," Applied Physics Letters 69, 2321-2323 (1996). 
130. Weber, M. J. Handbook of Optical Materials; CRC: New York, 2003

131. Q. Wu and X. -C. Zhang, "Ultrafast electro-optic field sensors," Appl. Phys. Lett. 68, 16041606 (1996).

132. Vodopyanov, K. L. Optical THz-wave generation with periodically-inverted GaAs. Laser \& Photon. Rev. 2, 11-25 (2008).

133. J. Guo, J.-J. Xie, D.-J. Li, G.-L. Yang, F. Chen, C.-R. Wang, L.-M. Zhang, Y. M. Andreev, K. A. Kokh, G. V. Lanskii, and V. A. Svetlichnyi, "Doped GaSe crystals for laser frequency conversion," Light: Science \& Applications 4, e362-e362 (2015).

134. C. Kübler, R. Huber, and A. Leitenstorfer, "Ultrabroadband terahertz pulses: generation and field-resolved detection," Semicond. Sci. Technol. 20, S128-S133 (2005).

135. P. Vohl, "Synthesis and crystal growth of $\mathrm{CdGeP}_{2}$," JEM 8, 517-528 (1979).

136. E. H. Turner, E. Buehler, and H. Kasper, "Electro-optic behavior and dielectric constants of $\mathrm{ZnGeP}_{2}$ and $\mathrm{CuGaS}_{2}$ " Phys. Rev. B 9, 558-561 (1974)

137. V. Petrov, F. Noack, I. Tunchev, P. Schunemann, and K. Zawilski, "The nonlinear coefficient d 36 of $\mathrm{CdSiP}_{2}, "$ in P. E. Powers, ed. (2009), p. 71970M.

138. J. Neu and C. A. Schmuttenmaer, "Tutorial: An introduction to terahertz time domain spectroscopy (THz-TDS)," Journal of Applied Physics 124, 231101 (2018).

139. T. J. Magnanelli and E. J. Heilweil, "Carrier mobility of silicon by sub-bandgap time-resolved terahertz spectroscopy," 28, (2020).

140. K. Song, Z. Tian, W. Zhang, and M. Wang, "Temperature-dependent birefringence of lithium triborate, LBO in the THz regime," Scientific Reports 7, 1-7 (2017).

141. X. Wu, C. Zhou, W. R. Huang, F. Ahr, and F. X. Kärtner, "Temperature dependent refractive index and absorption coefficient of congruent lithium niobate crystals in the terahertz range," Opt. Express, 23, 29729-29737 (2015).

142. B. G. Alberding, W. Robert Thurber, and E. J. Heilweil, "Direct comparison of time-resolved terahertz spectroscopy and Hall Van der Pauw methods for measurement of carrier conductivity and mobility in bulk semiconductors," Journal of the Optical Society of America B 34, 1392 (2017).

143.S. De and J. N. Coleman, "The effects of percolation in nanostructured transparent conductors," MRS Bulletin 36, 774-781 (2011).

144. T. R. Senty, S. K. Cushing, C. Wang, C. Matranga, and A. D. Bristow, "Inverting Transient Absorption Data to Determine Transfer Rates in Quantum Dot- $\mathrm{TiO}_{2}$ Heterostructures," J. Phys. Chem. C 119, 6337-6343 (2015). 
145. S. K. Cushing, J. Li, F. Meng, T. R. Senty, S. Suri, M. Zhi, M. Li, A. D. Bristow, and N. Wu, "Photocatalytic Activity Enhanced by Plasmonic Resonant Energy Transfer from Metal to Semiconductor," J. Am. Chem. Soc. 134, 15033-15041 (2012).

146. C. Klingshirn, Semiconductor Optics, 2nd ed. (Springer, Berlin, 2007).

147. R. Berera, R. van Grondelle, and J. T. M. Kennis, "Ultrafast transient absorption spectroscopy: principles and application to photosynthetic systems," Photosynth Res 101, 105118 (2009).

148. D. J. Vaughan and C. L. Corkhill, "Mineralogy of Sulfides," Elements 13, 81-87 (2017).

149. M. Grundmann, The Physics of Semiconductors: An Introduction Including Nanophysics and Applications, 3rd ed., Graduate Texts in Physics (Springer International Publishing, 2016).

150. V. G. Dmitriev, G. G. Gurzadyan, and D. N. Nikogosyan, Handbook of Nonlinear Optical Crystals, 3rd ed., Springer Series in Optical Sciences (Springer-Verlag, 1999).

151. K. Wiesauer and C. Jördens, "Recent Advances in Birefringence Studies at THz Frequencies," J Infrared Milli Terahz Waves 34, 663-681 (2013).

152. J. Lloyd-Hughes and T.-I. Jeon, "A Review of the Terahertz Conductivity of Bulk and NanoMaterials," Journal of Infrared, Millimeter, and Terahertz Waves 33, 871-925 (2012).

153.R. Ulbricht, E. Hendry, J. Shan, T. F. Heinz, and M. Bonn, "Carrier dynamics in semiconductors studied with time-resolved terahertz spectroscopy," Rev. Mod. Phys. 83, 543586 (2011).

154. R. Sooriyagoda, H. P. Piyathilaka, K. T. Zawilski, P. G. Schunemann, and A. D. Bristow, "Charge Carrier and Lattice Carrier Dynamics from Temperature-Dependent Terahertz Spectroscopy," in Frontiers in Optics / Laser Science (2020), Paper FM4C.6

155. R. Sooriyagoda, H. P. Piyathilaka, K. T. Zawilski, P. G. Schunemann, and A. D. Bristow, "Electronic and electron-lattice properties of the nonlinear chalcopyrite crystal CdGeP2," in OSA Advanced Photonics Congress (AP) 2020 (IPR, NP, NOMA, Networks, PVLED, PSC, SPPCom, SOF) (2020), Paper NpTu2E.7.

156. H. J. Joyce, J. L. Boland, C. L. Davies, S. A. Baig, and M. B. Johnston, "A review of the electrical properties of semiconductor nanowires: insights gained from terahertz conductivity spectroscopy," Semicond. Sci. Technol. 31, 103003 (2016).

157. L. Duvillaret, F. Garet, and J.- Coutaz, "A reliable method for extraction of material parameters in terahertz time-domain spectroscopy," IEEE Journal of Selected Topics in Quantum Electronics 2, 739-746 (1996).

158. R. Sooriyagoda, H. P. Piyathilaka, K. T. Zawilski, P. G. Schunemann, and A. D. Bristow, "Carrier transport and electron-lattice interactions of nonlinear optical crystals $\mathrm{CdGeP}_{2}$, ZnGeP 2 , and $\mathrm{CdSiP}_{2}$," J. Opt. Soc. Am. B, 38, 769-775 (2021). 
159. N. M. Ravindra, P. Ganapathy, and J. Choi, "Energy gap-refractive index relations in semiconductors - An overview," Infrared Physics \& Technology 50, 21-29 (2007).

160. J. A. McCaulley, V. M. Donnelly, M. Vernon, and I. Taha, "Temperature dependence of the near-infrared refractive index of silicon, gallium arsenide, and indium phosphide," Phys. Rev. B 49, 7408-7417 (1994).

161. Z. G. Hu and P. Hess, "Optical constants and thermo-optic coefficients of nanocrystalline diamond films at 30-500 C," Appl. Phys. Lett. 89, 081906 (2006).

162. T. Ruf, M. Cardona, C. S. J. Pickles, and R. Sussmann, "Temperature dependence of the refractive index of diamond up to 925 K," Phys. Rev. B 62, 16578-16581 (2000).

163.T. S. Moss, "A Relationship between the Refractive Index and the Infra-Red Threshold of Sensitivity for Photoconductors," Proc. Phys. Soc. B 63, 167-176 (1950).

164.N. M. Ravindra, S. Auluck, and V. K. Srivastava, "On the Penn Gap in Semiconductors," physica status solidi (b) 93, K155-K160 (1979).

165. P. J. L. Hervé and L. K. J. Vandamme, "Empirical temperature dependence of the refractive index of semiconductors," Journal of Applied Physics 77, 5476-5477 (1995).

166. V. Kumar and J. K. Singh, "Model for calculating the refractive index of different materials," APPL PHYS 48, 4 (2010).

167. Q. Liang, S. Wang, X. Tao, and T. Dekorsy, "Temperature dependence of free carriers and optical phonons in LiInSe 2 in the terahertz frequency regime," Phys. Rev. B 92, 144303 (2015).

168. U. Fano, "Effects of Configuration Interaction on Intensities and Phase Shifts," Phys. Rev. 124, 1866-1878 (1961).

169. Z.-L. Lv, H.-L. Cui, H. Wang, X.-H. Li, and G.-F. Ji, "Study of the vibrational, dielectric and infrared properties of CdSiP2 via first principles," Solid State Communications 246, 88-93 (2016).

170.H.-K. Nienhuys and V. Sundström, "Influence of plasmons on terahertz conductivity measurements," Appl. Phys. Lett. 87, 012101 (2005).

171.N. Smith, "Classical generalization of the Drude formula for the optical conductivity," Physical Review B 64, (2001).

172. T. L. Cocker, D. Baillie, M. Buruma, L. V. Titova, R. D. Sydora, F. Marsiglio, and F. A. Hegmann, "Microscopic origin of the Drude-Smith model," Phys. Rev. B 96, 205439 (2017).

173.N. A. Goryunova, A. S. Poplavnoi, Y. I. Polygalov, and V. A. Chaldyshev, "Energy Band Structure of Ternary Diamond-Like A2B4C-Type Semiconductors," physica status solidi (b) 39, 9-17 (1970). 
174.K. T. Zawilski, P. G. Schunemann, T. C. Pollak, D. E. Zelmon, N. C. Fernelius, and F. Kenneth Hopkins, "Growth and characterization of large CdSiP2 single crystals," Journal of Crystal Growth 312, 1127-1132 (2010).

175. E. M. Golden, N. C. Giles, E. Maniego, F. K. Hopkins, K. T. Zawilski, P. G. Schunemann, and L. E. Halliburton, "Identification of native defects (vacancies and antisites) in CdSiP2 crystals," Journal of Applied Physics 118, 185702 (2015).

176.N. C. Giles, L. Bai, M. M. Chirila, N. Y. Garces, K. T. Stevens, P. G. Schunemann, S. D. Setzler, and T. M. Pollak, "Infrared absorption bands associated with native defects in $\mathrm{ZnGeP}_{2}$," Journal of Applied Physics 93, 8975-8981 (2003).

177. P. P. Debye and E. M. Conwell, "Electrical Properties of N-Type Germanium," Phys. Rev. 93, 693-706 (1954).

178.C. M. Wolfe, G. E. Stillman, and W. T. Lindley, "Electron Mobility in High-Purity GaAs," Journal of Applied Physics 41, 3088-3091 (1970).

179. V. Kumar, A. K. Shrivastava, R. Banerji, and D. Dhirhe, "Debye temperature and melting point of ternary chalcopyrite semiconductors," Solid State Communications 149, 1008-1011 (2009).

180. S.-R. Zhang, "Theoretical study of the structural, elastic and thermodynamic properties of chalcopyrite ZnGeP2," Materials Science in Semiconductor Processing 9 (2015).

181. K. W. Böer and U. Pohl, Semiconductor Physics (Springer International Publishing, 2018).

182. R. Sooriyagoda, H. P. Piyathilaka, K. T. Zawilski, P. G. Schunemann, and A. D. Bristow, "Ultrafast Carrier Dynamics and Photoconductivity of the Chalcopyrite Crystals," in Frontiers in Optics / Laser Science (2020), Paper JW6B.11

183. G. M. Turner, M. C. Beard, and C. A. Schmuttenmaer, "Carrier Localization and Cooling in Dye-Sensitized Nanocrystalline Titanium Dioxide," J. Phys. Chem. B 106, 11716-11719 (2002).

184. W. Shockley and W. T. Read, "Statistics of the Recombinations of Holes and Electrons," Phys. Rev. 87, 835-842 (1952).

185. R. N. Hall, "Electron-Hole Recombination in Germanium," Phys. Rev. 87, 387-387 (1952).

186. W. Gerlach, H. Schlangenotto, and H. Maeder, "On the radiative recombination rate in silicon," physica status solidi (a) 13, 277-283 (1972).

187. P. T. Landsberg, "Trap-Auger recombination in silicon of low carrier densities," Applied Physics Letters 50, 745 (1998).

188. P. Uhd Jepsen, W. Schairer, I. H. Libon, U. Lemmer, N. E. Hecker, M. Birkholz, K. Lips, and M. Schall, "Ultrafast carrier trapping in microcrystalline silicon observed in optical pumpterahertz probe measurements," Applied Physics Letters 79, 1291-1293 (2001). 
189. T. S. Sosnowski, T. B. Norris, H. H. Wang, P. Grenier, J. F. Whitaker, and C. Y. Sung, "Highcarrier-density electron dynamics in low-temperature-grown GaAs," Appl. Phys. Lett. 70, 3245-3247 (1997).

190. J. Linnros, "Carrier lifetime measurements using free carrier absorption transients. I. Principle and injection dependence," Journal of Applied Physics 84, 275-283 (1998).

191. J. Linnros, "Carrier lifetime measurements using free carrier absorption transients. II. Lifetime mapping and effects of surface recombination," Journal of Applied Physics 84, 284291 (1998).

192. D. M. Caughey and R. E. Thomas, "Carrier mobilities in silicon empirically related to doping and field," Proceedings of the IEEE 55, 2192-2193 (1967).

193. Y. Dai, Q. Bu, R. Sooriyagoda, P. Tavadze, O. Pavlic, T. Lim, Y. Shen, A. Mamakhel, X. Wang, Y. Li, H. Niemantsverdriet, B. B. Iversen, F. Besenbacher, T. Xie, J. P. Lewis, A. D. Bristow, N. Lock, and R. Su, "Boosting Photocatalytic Hydrogen Production by Modulating Recombination Modes and Proton Adsorption Energy," J. Phys. Chem. Lett. 10, 5381-5386 (2019). 